\title{
A Convex Multi-Variable based Computational Framework for Multilayered Electro-Active Polymers
}

\author{
F. Marín ${ }^{\ddagger 1}$, J. Martínez-Frutos ${ }^{\dagger 2}$, R. Ortigosa ${ }^{\dagger 3}$, A. J. Gil ${ }^{\ddagger 4}$ \\ $\dagger$ Computational Mechanics and Scientific Computing Group, \\ Technical University of Cartagena, Campus Muralla del Mar, 30202, Cartagena (Murcia), Spain \\ $\ddagger$ Zienkiewicz Centre for Computational Engineering, College of Engineering \\ Swansea University, Bay Campus, SA1 8EN, United Kingdom
}

\begin{abstract}
This paper presents a novel computational framework for the in silico analysis of rank-one multilayered electro-active polymer composites exhibiting complex deformation patterns. The work applies the principles of rank-n homogenisation in the context of extremely deformable dielectric elastomers actuated beyond the onset of geometrical instabilities. Following previous work by the authors [31, 52, 53], Convex Multi-Variable (CMV) energy density functionals are used to describe the physics of the individual microscopic constituents, which is shown to guarantee $a b$ initio the existence of solutions for the microstructure problem, described in terms of the so-called deformation gradient and electric displacement amplitude vectors. The high nonlinearity of the quasi-static electro-mechanical problem is resolved via a monolithic multi-scale Newton-Raphson scheme, which is enhanced with a tailor-made arc length technique, used to circumvent the onset of geometrical instabilities. A tensor cross product operation between vectors and tensors and an additive decomposition of the micro-scale deformation gradient (in terms of macro-scale and fluctuation components) are used to considerably reduce the complexity of the algebra. The possible loss of ellipticity of the homogenised constitutive model is strictly monitored through the minors of the homogenised acoustic tensor. A series of numerical examples is presented in order to demonstrate the effect that the volume fraction, the contrast and the material properties, as well as the level of deformation and electric field, have upon the response of the composites when subjected to large three dimensional stretching, bending and torsion, including the possible development of wrinkling.
\end{abstract}

Keywords: finite element method, nonlinear electro-elasticity, composite materials, rank-one laminates

\section{Introduction}

Electro-Active Polymers (EAPs) have emerged as a class of smart materials capable of displaying significant change in shape in response to electrical stimuli. EAPs have been thoroughly studied over the years $[3,18,41,56]$ with main properties being their high resilience, short coupled response time and their capacity to sustain large strains. EAPs can be broadly classified [12] into Ionic EAPs, activated by transport of ions, and Electronic EAPs, actuated through the application of an electric field.

\footnotetext{
${ }^{1}$ Corresponding author: francisco.marin@swansea.ac.uk

${ }^{2}$ Corresponding author: jesus.martinez@upct.es

${ }^{3}$ Corresponding author: rogelio.ortigosa@upct.es

${ }^{4}$ Corresponding author: a.j.gil@swansea.ac.uk
} 
Dielectric elastomers (DEs) are recognised as one of the most popular Electronic EAPs [43, 55, 57] due to their outstanding actuation capabilities (i.e., light weight, fast response time, flexibility, low stiffness properties), which makes them ideal for their use as soft robots $[3,4,10,13,14,41$, $46,51,62]$ or flexible energy generators [42, 48]. However, an extremely large electric field is generally required in order to access the large actuation regime in DEs, an aspect that very often places them on the risk of electromechanical instabilities or even electrical breakdown [5].

With the aim of reducing the high operational voltage required for actuation in DEs, some authors have advocated for the design of composite-based DEs [37, 38, 78], typically combining an ultra-soft and low-permittivity elastomer matrix with a stiffer and high-permittivity inclusion randomly distributed in the form of fibres or particles [36, 58]. Experimental studies [37, 70] have demonstrated a significant enhancement in the electromechanical coupling performance of DE composites, thus reducing the operational voltage required for actuation. Due to the continuous improvement in layer-by-layer fabrication techniques [26], multi-layered laminated DE composites have gained considerable momentum over alternative DE composites.

From the modelling point of view, Li et al. [44, 45] highlighted the strong nonlinearity of the electromechanical coupling in layered DE composites and deBotton et al. [20] demonstrated that the contrast between the properties of the composite constituents is one of their critical design factors. More recently, Tian et al. [72] demonstrated that the actuation performance of a DE composite subjected to simple in-plane stretching can be amplified by several orders of magnitude with respect to that of a single-phase DE, even in the linearised regime (i.e., in the proximity of the origin of deformations and electric fields). Gei et al. [28] confirmed the same finding in the moderate strain regime, where an optimisation analysis in terms of the contrast and volume fraction of the DE composite constituents was carried out. For the case of composites different to laminated EAPs (out of the scope of this paper), Shrimali et al. [68] have presented explicit analytical formulae (analytical homogenisation) for the behaviour of porous-type materials, and Göküzüm et al. [33, 34] have made use of Fourier transforms and artificial neuronetworks.

Other works presenting modelling studies of layered DE composites are those in $[5,18,29$, 61, 72]. Specifically, Bertoldi and Gei [5] and Rudykh et al. [60, 61, 63] focused on the study of instabilities (namely, geometrical buckling, microscopic modes, macroscopic modes, dielectric breakdown) in rank-one DE laminated composites, whereas in [26, 66, 67] the authors focus on the study of wave propagation and its application into phononic band gaps. Typically, small amplitude shear wave propagation is studied in DE layered media comprised of two incompressible isotropic electro-elastic phases, and the effect of the contrast of the constituents on the slowness (i.e., inverse of the wave speed) is analysed. A common feature of all of the above modelling studies lies in the search for closed-form solutions, which are achieved for the case of simple in-plane stretching deformation scenarios, primarily described by plane strain and exact incompressibility, in conjunction with the use of ideal dielectric neo-Hookean phases. As a result, one of the objectives of this paper is the study of more realistic three-dimensional deformation scenarios (namely, combined bending/torsion/stretching), way beyond the onset of geometrical instabilities and without the need to resort to any simplifications in the kinematics of the problem (e.g., plane strain, exact incompressibility).

With the aim of modelling more complex deformation scenarios, the use of computational methods constructed on the basis of variational principles is nowadays acknowledged as the preferred method of choice. Building upon the early works of Toupin [73, 74] and those of Dorfmann, Ogden, MacMeeking, Suo and co-workers in [21, 22, 49, 71], recent contributions in the field of computational electro-mechanics can be found in $[6,23,40,75,76]$. In these works, the constitutive behaviour of a single-phase electro-mechanical material is encoded within a carefully (phenomenologically) defined energy functional which depends upon appropriate strain measures, a Lagrangian electric variable and, if dissipative effects are considered, an electromechanical internal variable [47]. In previous publications [31, 52, 54, 59], the authors put forward a new computa- 
tional framework for single-phase reversible electro-mechanics, where the existence of solutions is always ensured via the selection of objective (frame-invariant) and Convex Multi-Variable (CMV) energy functionals, that is, convex with respect to the minors of the deformation gradient tensor $\{\boldsymbol{F}, \boldsymbol{H}, J\}$, the Lagrangian electric displacement $\boldsymbol{D}_{0}$, and the spatial electric displacement $\boldsymbol{d}=\boldsymbol{F} \boldsymbol{D}_{0}$. CMV energy functionals (generally referred to as polyconvex [69]) guarantee ellipticity [31] in the quasi-static case and hyperbolicity in the dynamic case [52], thus precluding anomalous mesh dependency effects. Accordingly, a second objective of this paper is the use of CMV-based energy functionals (at the micro level) in order to describe each of the individual microscopic constituents of the multi-layered DE composite, adding further complexity to that of the standard incompressible ideal dielectric elastomer.

As a result, from the constitutive modelling standpoint, this paper will apply the principles of rank-n homogenisation of CMV phases in the context of extremely deformable dielectric elastomers actuated well beyond the onset of geometrical instabilities. From the numerical standpoint, a novel computational framework for the finite element analysis of rank-one multi-layered electroactive polymer composites will be presented. The high nonlinearity of the quasi-static electromechanical problem will be addressed via a monolithic multi-scale Newton-Raphson scheme, with an arc length technique used to bypass geometrical instabilities. As a further novelty, a tensor cross product operation between vectors and tensors $[7,8]$ and an additive decomposition of the micro-scale deformation gradient (in terms of macro-scale and fluctuation components) will be used to considerably reduce the complexity of the algebra.

The outline of this paper is as follows. Section 2 describes the necessary elements of nonlinear continuum electro-mechanics. Section 3 revisits the concept of Multi-Variable Convexity as a basis for the constitutive models for the description of the individual microscopic components in multilayered DE composites. Section 4 presents the homogenisation theory applied to rank-one electromechanical laminates and demonstrates the necessary conditions of existence for the microscopic and macroscopic problems to be solved. Section 5 succinctly describes the variational principles and the finite element implementation method used in this work. Section 6 presents a series of numerical examples in order to assess the capabilities of the new computational framework. Specifically, in a first example, a local analysis is conducted at a quadrature (Gauss point) level where the effect of volume fraction, contrast and laminate orientation upon electro-mechanical performance, macroscopic stability and shear wave speed propagation is presented. In a second example, complex three-dimensional bending/torsion/stretching combined modes of deformation are studied for a soft robot actuator, monitoring macroscopic stability. In a third example, the onset of first and second order buckling (as referred to in [32]) is explored in a prototypical laboratory configuration. Eventually, Section 7 provides some concluding remarks.

\section{Nonlinear continuum electro-mechanics}

\subsection{Kinematics: motion and deformation}

Let us consider the motion of an Electro-Active Polymer (EAP) with reference configuration given by the open bounded set $\mathcal{B}_{0} \subset \mathbb{R}^{3}$ with boundary $\partial \mathcal{B}_{0}$ and unit outward normal $\boldsymbol{N}$. After the motion, the EAP occupies a deformed configuration given by the open bounded set $\mathcal{B} \subset \mathbb{R}^{3}$ with boundary $\partial \mathcal{B}$ and unit outward normal $\boldsymbol{n}$. The motion of the EAP is defined by a deformation mapping $\boldsymbol{\phi}$ linking material particles $\boldsymbol{X} \in \mathcal{B}_{0}$ to the deformed configuration $\boldsymbol{x} \in \mathcal{B}$ as

$$
\begin{aligned}
\phi: \mathcal{B}_{0} \subset \mathbb{R}^{3} & \rightarrow \mathcal{B} \subset \mathbb{R}^{3} \\
\boldsymbol{X} & \mapsto \boldsymbol{x}=\boldsymbol{\phi}(\boldsymbol{X}) .
\end{aligned}
$$

This motion is represented in Figure 1. Associated with $\boldsymbol{\phi}(\boldsymbol{X})$, the deformation gradient 
tensor $\boldsymbol{F}[9,16,35]$ is defined as $^{5}$

$$
\boldsymbol{F}=\nabla_{0} \boldsymbol{\phi}(\boldsymbol{X}) ; \quad F_{i I}=\frac{\partial \phi_{i}}{\partial X_{I}}=\partial_{X_{I}}\left(\phi_{i}\right)
$$

Associated with $\boldsymbol{F}$, its co-factor $\boldsymbol{H}$ and its Jacobian $J[8,15]$ are defined as

$$
\boldsymbol{H}=\frac{1}{2} \boldsymbol{F} \times \boldsymbol{F} ; \quad J=\frac{1}{3} \boldsymbol{H}: \boldsymbol{F},
$$

with $(\boldsymbol{A} \times \boldsymbol{B})_{i I}=\mathcal{E}_{i j k} \mathcal{E}_{I J K} A_{j J} B_{k K}, \forall \boldsymbol{A}, \boldsymbol{B} \in \mathbb{R}^{3 \times 3}$, where $\mathcal{E}_{i j k}$ (or $\mathcal{E}_{I J K}$ ) symbolises the third-order alternating tensor components and the use of repeated indices implies summation ${ }^{6}$.

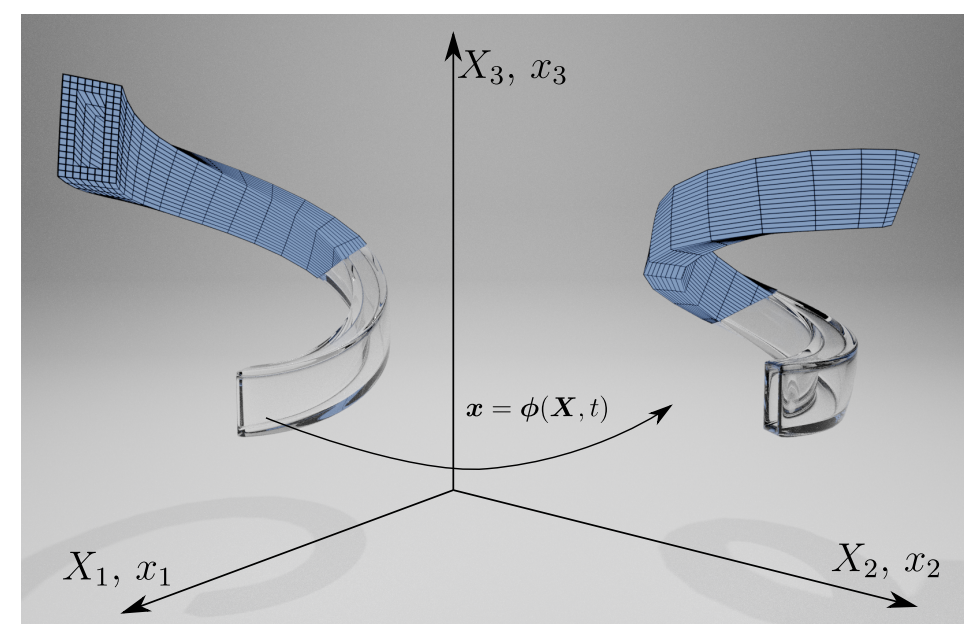

Figure 1: Deformation mapping $\boldsymbol{\phi}(\boldsymbol{X})$.

\subsection{Governing equations in nonlinear electromechanics}

In the absence of inertia and magnetic effects, the system of partial differential equations governing the behaviour of the EAP comprises the conservation of linear momentum and the compatibility equation $(2)_{a}$ along with the quasi-static version of the Gauss's and Faraday's laws. The complete Boundary Value problem can be recast in a Total Lagragian formalism as

$$
\begin{aligned}
& \boldsymbol{F}=\nabla_{0} \phi ; \quad \text { in } \mathcal{B}_{0} ; \quad \boldsymbol{E}_{0}=-\nabla_{0} \varphi ; \quad \text { in } \mathcal{B}_{0} ; \\
& \operatorname{DIVP}\left(\boldsymbol{F}, \boldsymbol{E}_{0}\right)+\boldsymbol{f}_{0}=\mathbf{0} ; \quad \text { in } \mathcal{B}_{0} ; \quad \operatorname{DIV}_{0}\left(\boldsymbol{F}, \boldsymbol{E}_{0}\right)-\rho_{0}=0 ; \quad \text { in } \mathcal{B}_{0} \\
& \boldsymbol{P} N=\boldsymbol{t}_{0} ; \quad \text { on } \partial_{\boldsymbol{t}} \mathcal{B}_{0} \\
& \phi=\bar{\phi} ; \quad \text { on } \partial_{\phi} \mathcal{B}_{0} ;
\end{aligned}
$$

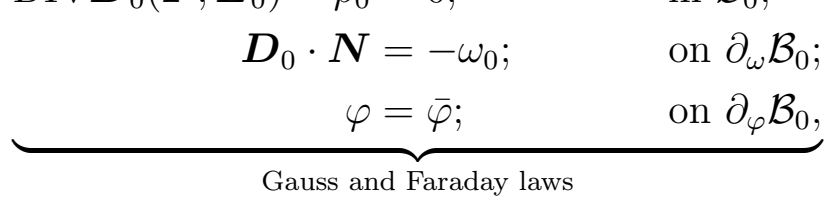

where $\boldsymbol{f}_{0}$ represents a body force per unit undeformed volume $\mathcal{B}_{0} ; \boldsymbol{t}_{0}$, the traction force per unit undeformed area on $\partial_{t} \mathcal{B}_{0} \subset \partial \mathcal{B}_{0} ; \overline{\boldsymbol{\phi}}$, the value of the Dirichlet boundary condition on $\partial_{\phi} \mathcal{B}_{0} \subset \partial \mathcal{B}_{0}$, with $\partial_{t} \mathcal{B}_{0} \cup \partial_{\phi} \mathcal{B}_{0}=\partial \mathcal{B}_{0}$ and $\partial_{t} \mathcal{B}_{0} \cap \partial_{\phi} \mathcal{B}_{0}=\emptyset$. Furthermore, $\rho_{0}$ represents an electric volume charge per unit of undeformed volume $\mathcal{B}_{0}$, and $\omega_{0}$ an electric surface charge per unit of undeformed area $\partial_{\omega} \mathcal{B}_{0} \subset \partial \mathcal{B}_{0}$. In addition, $\boldsymbol{E}_{0}$ is the Lagrangian electric field vector; $\varphi: \mathcal{B}_{0} \rightarrow \mathbb{R}$, the scalar

\footnotetext{
${ }^{5}$ Lower case indices $\{i, j, k\}$ will be used to represent the spatial configuration, whereas capital case indices $\{I, J, K\}$ will be used to represent the material description.

${ }^{6}$ In addition, throughout the paper, the symbol $(\cdot)$ indicates the scalar product or contraction of a single index $\boldsymbol{a} \cdot \boldsymbol{b}=a_{i} b_{i}$; the symbol $(:)$, double contraction of two indices $\boldsymbol{A}: \boldsymbol{B}=A_{i j} B_{i j}$; the symbol $(\times)$, the cross product between vectors $(\boldsymbol{a} \times \boldsymbol{b})_{i}=\mathcal{E}_{i j k} a_{j} b_{k}$; and the symbol $(\otimes)$, the outer or dyadic product $(\boldsymbol{a} \otimes \boldsymbol{b})_{i j}=a_{i} b_{j}$.
} 
electric potential; and $\partial_{\varphi} \mathcal{B}_{0}$, the part of the boundary $\partial \mathcal{B}_{0}$ where essential electric potential boundary conditions are applied so that $\partial_{\omega} \mathcal{B}_{0} \cup \partial_{\varphi} \mathcal{B}_{0}=\partial \mathcal{B}_{0}$ and $\partial_{\omega} \mathcal{B}_{0} \cap \partial_{\varphi} \mathcal{B}_{0}=\emptyset$. Finally, $\boldsymbol{P}\left(\boldsymbol{F}, \boldsymbol{E}_{0}\right)$ and $\boldsymbol{D}_{0}\left(\boldsymbol{F}, \boldsymbol{E}_{0}\right)$ represent the first Piola-Kirchhoff stress tensor and the Lagrangian electric displacement field, respectively, which, in the reversible case, depend upon $\boldsymbol{F}$ and $\boldsymbol{E}_{0}$.

\section{Constitutive equations in nonlinear electro-elasticity: phenomenological macro- scopic models}

The scope of this paper is on analytical homogenisation of layered EAP composites. However, we devote the current section to the simpler case where a macroscopic phenomenological electromechanical model is available, in order to introduce some key concepts which will be exploited in subsequent sections.

\subsection{Multi-variable convexity}

In the case of reversible electro-elasticity, the internal energy density $e$ per unit of undeformed volume can be defined in terms of the deformation and the electric displacement field, namely $e=e\left(\boldsymbol{F}, \boldsymbol{D}_{0}\right)[50]$. In addition, the requirement of objectivity implies that the internal energy $e$ can be re-expressed in terms of a set of objective arguments (namely, $\boldsymbol{D}_{0}$ and $\boldsymbol{C}=\boldsymbol{F}^{T} \boldsymbol{F}$ ) as $e\left(\boldsymbol{F}, \boldsymbol{D}_{0}\right)=\widetilde{e}\left(\boldsymbol{C}, \boldsymbol{D}_{0}\right)$, where $e$ and $\widetilde{e}$ denote alternative functional representations of the same internal energy density. From standard thermodynamical arguments [31], the first directional derivative of the internal energy gives the first Piola-Kirchhoff stress tensor $\boldsymbol{P}$ and the electric field $\boldsymbol{E}_{0}$ as

$$
D e\left[\delta \boldsymbol{F}, \delta \boldsymbol{D}_{0}\right]=\boldsymbol{P}: \delta \boldsymbol{F}+\boldsymbol{E}_{0} \cdot \delta \boldsymbol{D}_{0}
$$

with

$$
\boldsymbol{P}\left(\boldsymbol{F}, \boldsymbol{D}_{0}\right)=\partial_{\boldsymbol{F}} e ; \quad \boldsymbol{E}_{0}\left(\boldsymbol{F}, \boldsymbol{D}_{0}\right)=\partial_{\boldsymbol{D}_{0}} e
$$

where $\partial_{\boldsymbol{A}}(\bullet)$ denotes the partial derivative of $(\bullet)$ with respect to the field $\boldsymbol{A}$. The second directional derivative of the internal energy yields the Hessian operator $\left[\mathbb{H}_{e}\right]$, expressed in terms of an elasticity tensor $\mathcal{C}_{e} \in \mathbb{R}^{3 \times 3 \times 3 \times 3}$, the third order tensor $\mathcal{Q} \in \mathbb{R}^{3 \times 3 \times 3}$ and the inverse of the dielectric tensor $\boldsymbol{\theta} \in \mathbb{R}^{3 \times 3}$, namely

$$
D^{2} e\left[\delta \boldsymbol{F}, \delta \boldsymbol{D}_{0} ; \delta \boldsymbol{F}, \delta \boldsymbol{D}_{0}\right]=\left[\delta \boldsymbol{F}: \delta \boldsymbol{D}_{0} \cdot\right]\left[\mathbb{H}_{e}\right]\left[\begin{array}{l}
: \delta \boldsymbol{F} \\
\delta \boldsymbol{D}_{0}
\end{array}\right] ; \quad\left[\mathbb{H}_{e}\right]=\left[\begin{array}{cc}
\mathcal{C}_{e} & \mathcal{Q}^{T} \\
\mathcal{Q} & \boldsymbol{\theta}
\end{array}\right]
$$

where

$$
\mathcal{C}_{e}\left(\boldsymbol{F}, \boldsymbol{D}_{0}\right)=\partial_{\boldsymbol{F} \boldsymbol{F}}^{2} e ; \quad \mathcal{Q}\left(\boldsymbol{F}, \boldsymbol{D}_{0}\right)=\partial_{\boldsymbol{D}_{0} \boldsymbol{F}}^{2} e ; \quad \boldsymbol{\theta}\left(\boldsymbol{F}, \boldsymbol{D}_{0}\right)=\partial_{\boldsymbol{D}_{0} \boldsymbol{D}_{0}}^{2} e
$$

with $\left(\mathcal{Q}^{T}\right)_{j J I}=(\mathcal{Q})_{I j J}$. To ensure existence of solutions in the vicinity of the origin, namely when $\boldsymbol{F} \approx \boldsymbol{I}$ (with $\boldsymbol{I}$ the second order identity matrix) and $\boldsymbol{D}_{0} \approx \mathbf{0}$, the internal energy functional must be (strictly) convex, i.e.,

$$
\left.D^{2} e\left[\delta \boldsymbol{F}, \delta \boldsymbol{D}_{0} ; \delta \boldsymbol{F}, \delta \boldsymbol{D}_{0}\right]\right|_{\boldsymbol{F}=\boldsymbol{I}, \boldsymbol{D}_{0}=\mathbf{0}}>0 ; \quad \forall \delta \boldsymbol{F}, \delta \boldsymbol{D}_{0}
$$

which requires positive definiteness of $\mathcal{C}_{e}$ and $\boldsymbol{\theta}$, independently, and further restrictions on the form that the third-order tensor $\mathcal{Q}$ can adopt ${ }^{7}$. However, convexity away from the origin is not a physical restriction as it precludes buckling in the purely mechanical context, as well as the possibility of voltage-induced buckling, inherent to soft dielectric materials. Alternatively, the most well-accepted mathematical restriction is the so-called ellipticity or rank-one convexity condition, closely related to the concept of material stability, which can be deduced as a particularisation of

\footnotetext{
${ }^{7}$ Sufficient smoothness of the internal energy $e$ is assumed, so that $\left[\mathbb{H}_{e}\right]$ is well defined.
} 
the convexity condition for the case when $\delta \boldsymbol{F}=\boldsymbol{u} \otimes \boldsymbol{V}$ and $\delta \boldsymbol{D}_{0}=\boldsymbol{V}_{\perp}$, with $\boldsymbol{V}_{\perp} \cdot \boldsymbol{V}=0$ and $\boldsymbol{u}, \boldsymbol{V}, \boldsymbol{V}_{\perp}$ any arbitrary non-zero vectors. Thus, (strict) ellipticity implies

$$
D^{2} e\left[\boldsymbol{u} \otimes \boldsymbol{V}, \boldsymbol{V}_{\perp} ; \boldsymbol{u} \otimes \boldsymbol{V}, \boldsymbol{V}_{\perp}\right]=\left[\boldsymbol{u} \otimes \boldsymbol{V}: \boldsymbol{V}_{\perp} \cdot\right]\left[\mathbb{H}_{e}\right]\left[\begin{array}{c}
: \boldsymbol{u} \otimes \boldsymbol{V} \\
\boldsymbol{V}_{\perp}
\end{array}\right]>0 ; \quad \forall \boldsymbol{u}, \boldsymbol{V}, \boldsymbol{V}_{\perp} .
$$

Remark 1. Notice that the vector $\boldsymbol{V}_{\perp}$ in (10) is orthogonal to $\boldsymbol{D}_{0}$. The reason for this choice has its roots in the analysis of the hyperbolicity of the system of PDEs in (4) in the dynamic context. In this case, it is customary to express the fields $\boldsymbol{\phi}$ and $\boldsymbol{D}_{0}$ as a perturbation with respect to equilibrium states $\phi^{\mathrm{eq}}$ and $\boldsymbol{D}_{0}^{\mathrm{eq}}$, respectively, by means of the addition of travelling wave functions as

$$
\boldsymbol{\phi}=\boldsymbol{\phi}^{\mathrm{eq}}+\boldsymbol{u} \hat{\phi}(\boldsymbol{X} \cdot \boldsymbol{V}-c t) ; \quad \boldsymbol{D}_{0}=\boldsymbol{D}_{0}^{\mathrm{eq}}+\boldsymbol{V}_{\perp} \hat{\phi}(\boldsymbol{X} \cdot \boldsymbol{V}-c t),
$$

where $\boldsymbol{V}$ represents the polarisation vector of the travelling wave and $c$ the associated speed of propagation of the perturbation with amplitudes $\boldsymbol{u}$ and $\boldsymbol{V}_{\perp}$. Introduction of the ansatz for $\boldsymbol{D}_{0}$ into equation (4) reveals that

$$
\operatorname{DIV} \boldsymbol{D}_{0}-\rho_{0}=\underbrace{\mathrm{DIV} \boldsymbol{D}_{0}^{\mathrm{eq}}-\rho_{0}}_{=0}+\left(\boldsymbol{V}_{\perp} \cdot \boldsymbol{V}\right) \hat{\phi}^{\prime}(\boldsymbol{X} \cdot \boldsymbol{V}-c t)=0,
$$

and hence, $\boldsymbol{V}_{\perp}$ must be orthogonal to $\boldsymbol{V}$.

Motivated by considerations of material stability, Gil and Ortigosa [31, 52-54] extended the concept of polyconvexity $[1,2,39,64,65]$ from elasticity to electro-magneto-elasticity and proposed new convexity restrictions on the internal energy, postulating a Convex Multi-Variable (CMV) definition as

$$
e\left(\boldsymbol{F}, \boldsymbol{D}_{0}\right)=W(\mathcal{V}) ; \quad \mathcal{V}=\left\{\boldsymbol{F}, \boldsymbol{H}, J, \boldsymbol{D}_{0}, \boldsymbol{d}\right\} ; \quad \boldsymbol{d}=\boldsymbol{F} \boldsymbol{D}_{0}
$$

where $W$ must be a convex function with respect to the extended set $\mathcal{V}$. As shown in Remark 1 below, the new extended set of convex restrictions proved to be a sufficient condition for the satisfaction of the ellipticity condition (10). Shortly after the work in [31], Šilhavý [69] proved that multi-variable convexity, or $\mathcal{A}$-polyconvexity, as he denoted it in his work, in conjunction with suitable growth conditions, ensures existence of minimisers in nonlinear electro-magnetoelasticity.

Remark 2. As shown in Reference [31], the second directional derivative of the internal energy can be equivalently expressed in terms of its extended representation $W(\mathcal{V})$ as

$$
\begin{aligned}
D^{2} e\left[\delta \boldsymbol{F}, \delta \boldsymbol{D}_{0} ; \delta \boldsymbol{F}, \delta \boldsymbol{D}_{0}\right] & =D^{2} W[\delta \mathcal{V} ; \delta \mathcal{V}]+\left(\partial_{\boldsymbol{H}} W+\partial_{J} W \boldsymbol{F}\right):(\delta \boldsymbol{F} \times \delta \boldsymbol{F})+2 \partial_{\boldsymbol{d}} W \cdot \delta \boldsymbol{F} \delta \boldsymbol{D}_{0} \\
& =[\mathbb{S}]^{T}\left[\mathbb{H}_{W}\right][\mathbb{S}]+\left(\partial_{\boldsymbol{H}} W+\partial_{J} W \boldsymbol{F}\right):(\delta \boldsymbol{F} \times \delta \boldsymbol{F})+2 \partial_{\boldsymbol{d}} W \cdot \delta \boldsymbol{F} \delta \boldsymbol{D}_{0}
\end{aligned}
$$

with $[\mathbb{S}]$ and the extended Hessian operator $\left[\mathbb{H}_{W}\right]$ defined as

$$
[\mathbb{S}]=\left[\begin{array}{c}
: \delta \boldsymbol{F} \\
:(\delta \boldsymbol{F} \times \boldsymbol{F}) \\
\delta \boldsymbol{F}: \boldsymbol{H} \\
\cdot \delta \boldsymbol{D}_{0} \\
\cdot\left(\delta \boldsymbol{F} \boldsymbol{D}_{0}+\boldsymbol{F} \delta \boldsymbol{D}_{0}\right)
\end{array}\right] ; \quad\left[\mathbb{H}_{W}\right]=\left[\begin{array}{ccccc}
\partial_{\boldsymbol{F} F}^{2} W & \partial_{\boldsymbol{F} \boldsymbol{H}}^{2} W & \partial_{\boldsymbol{F} J}^{2} W & \partial_{\boldsymbol{F} \boldsymbol{D}_{0}}^{2} W & \partial_{\boldsymbol{F} \boldsymbol{d}} W \\
\partial_{\boldsymbol{H} \boldsymbol{F}}^{2} W & \partial_{\boldsymbol{H}_{\boldsymbol{H}}}^{2} W & \partial_{\boldsymbol{H} J}^{2} W & \partial_{\boldsymbol{H} \boldsymbol{D}_{0}}^{2} W & \partial_{\boldsymbol{H} \boldsymbol{d}}^{2} W \\
\partial_{J \boldsymbol{F}}^{2} W & \partial_{J \boldsymbol{H}}^{2} W & \partial_{J J}^{2} W & \partial_{J \boldsymbol{D}_{0}}^{2} W & \partial_{J \boldsymbol{d}}^{2} W \\
\partial_{\boldsymbol{D}_{0} \boldsymbol{F}}^{2} W & \partial_{\boldsymbol{D}_{0} \boldsymbol{H}^{2}}^{2} W & \partial_{\boldsymbol{D}_{0}}^{2} W & \partial_{\boldsymbol{D}_{0} \boldsymbol{D}_{0}}^{2} W & \partial_{\boldsymbol{D}_{0} \boldsymbol{d}} W \\
\partial_{\boldsymbol{d} \boldsymbol{F}}^{2} W & \partial_{\boldsymbol{d} \boldsymbol{H}}^{2} W & \partial_{\boldsymbol{d} J}^{2} W & \partial_{\boldsymbol{d} \boldsymbol{D}_{0}} W & \partial_{\boldsymbol{d} \boldsymbol{d}} W
\end{array}\right] .
$$

As presented in [54], it is possible to relate the components of the Hessian $\left[\mathbb{H}_{W}\right]$ to those of the Hessian $\left[\mathbb{H}_{e}\right]$ via appropriate algebraic transformations. Replacing now $\delta \boldsymbol{F}$ and $\delta \boldsymbol{D}_{0}$ in (14) 
with $\delta \boldsymbol{F}=\boldsymbol{u} \otimes \boldsymbol{V}$ and $\delta \boldsymbol{D}_{0}=\boldsymbol{V}_{\perp}$, respectively, permits to cancel the last two terms (also known as geometric terms) on the right-hand side of (14), and leads to

$$
D^{2} e\left[\boldsymbol{u} \otimes \boldsymbol{V}, \delta \boldsymbol{V}_{\perp} ; \boldsymbol{u} \otimes \boldsymbol{V}, \delta \boldsymbol{V}_{\perp}\right]=[\mathbb{S}]^{T}\left[\mathbb{H}_{W}\right][\mathbb{S}]
$$

Equation (16) clearly illustrates that CMV internal energy functionals, characterised by a positive definite Hessian operator $\left[\mathbb{H}_{W}\right]$, guarantee positiveness of the left-hand side of equation (16), and hence the fulfillment of the strong ellipticity condition in (10).

\subsection{Simple examples of convex multi-variable electro-mechanical constitutive models}

In the definition of electromechanical constitutive models for dielectric elastomers, it is customary to propose an additive decomposition of the internal energy $e\left(\boldsymbol{F}, \boldsymbol{D}_{0}\right)$ into a purely mechanical contribution and a coupled electromechanical contribution $[11,39,75]$ as

$$
e\left(\boldsymbol{F}, \boldsymbol{D}_{0}\right)=e_{m}(\boldsymbol{F})+e_{e m}\left(\boldsymbol{F}, \boldsymbol{D}_{0}\right) .
$$

The simplest expression for the electromechanical contribution corresponds to that of an ideal dielectric elastomer, defined as

$$
e_{e m}\left(\boldsymbol{F}, \boldsymbol{D}_{0}\right)=W_{e m}(J, \boldsymbol{d})=\frac{1}{2 \epsilon_{r} \epsilon_{0} J} I I_{\boldsymbol{d}}
$$

where $\epsilon_{0}$ represents the vacuum permittivity, with $\epsilon_{0}=8.8541 \times 10^{-12} \mathrm{C}^{2} \mathrm{~N}^{-1} \mathrm{~m}^{-2} ; \epsilon_{r}$, the relative permittivity; and $I I_{\bullet}$, the square of the Euclidean norm. Alternative CMV electromechanical contributions can be defined as

$$
e_{e m}\left(\boldsymbol{F}, \boldsymbol{D}_{0}\right)=\frac{g_{1}(J)}{\epsilon_{1}} I I_{\boldsymbol{d}}+\frac{g_{2}(J)}{\epsilon_{2}} I I_{\boldsymbol{D}_{0}}
$$

with $\left\{\epsilon_{1}, \epsilon_{2}\right\}$ material parameters with units of electric permittivity. It can be shown that sufficient conditions for $g_{1}(J)$ and $g_{2}(J)$ to ensure that $e_{e m}\left(\boldsymbol{F}, \boldsymbol{D}_{0}\right)$ is CMV compliant are

$$
g_{i}(J) g_{i}^{\prime \prime}(J) \geq 2\left(g_{i}^{\prime}(J)\right)^{2}, \forall J>0 ; \quad g_{i}^{\prime \prime}(J)>0, g_{i}(J)>0, \forall J>0 ; \quad i=\{1,2\} .
$$

A possible example complying with above conditions (20) is

$$
g_{i}(J)=J^{\alpha} ; \quad \alpha \in[-1,0) .
$$

Remark 3. We derive the conditions shown in equation (20). For that, and without loss of generality, we consider only the first contribution in (19), excluding the constant $\epsilon_{1}$ for simplicity. Therefore, we consider the following simplified electromechanical contribution

$$
e_{e m}=g_{1}(J) I I_{\boldsymbol{d}}
$$

The second directional derivative of $e_{e m}$ in (22) with respect to its arguments yields

$$
D^{2} e_{e m}[\delta J, \delta \boldsymbol{d} ; \delta J, \delta \boldsymbol{d}]=g^{\prime \prime}(J) \delta J^{2} I I_{\boldsymbol{d}}+2 I I_{\delta \boldsymbol{d}} g(J)+4 g^{\prime}(J) \delta J(\boldsymbol{d} \cdot \delta \boldsymbol{d}) .
$$

Introduction of the following notation $\boldsymbol{a}=\sqrt{2 g(J)} \delta \boldsymbol{d}$, and $\boldsymbol{b}=\sqrt{g^{\prime \prime}(J)} \delta J \boldsymbol{d}$ into (23) yields the following expression

$$
D^{2} e_{e m}[\delta J, \delta \boldsymbol{d} ; \delta J, \delta \boldsymbol{d}]=I I_{\boldsymbol{a}}+I I_{\boldsymbol{b}}+4 g^{\prime}(J) \delta J(\boldsymbol{d} \cdot \delta \boldsymbol{d}),
$$


which can be conveniently manipulated as

$$
\begin{aligned}
D^{2} e_{e m}[\delta J, \delta \boldsymbol{d} ; \delta J, \delta \boldsymbol{d}] & =I I_{\boldsymbol{a}}+I I_{\boldsymbol{b}}-2|\boldsymbol{a} \cdot \boldsymbol{b}|+2|\boldsymbol{a} \cdot \boldsymbol{b}|+4 g^{\prime}(J) \delta J(\boldsymbol{d} \cdot \delta \boldsymbol{d}) \\
& =(|\boldsymbol{a}|-|\boldsymbol{b}|) \cdot(|\boldsymbol{a}|-|\boldsymbol{b}|)+2|\boldsymbol{a} \cdot \boldsymbol{b}|+4 g^{\prime}(J) \delta J(\boldsymbol{d} \cdot \delta \boldsymbol{d}) \\
& \geq 2|\boldsymbol{a} \cdot \boldsymbol{b}|-4\left|g^{\prime}(J)\right||\delta J(\boldsymbol{d} \cdot \delta \boldsymbol{d})| .
\end{aligned}
$$

Making use of the definition of the vectors $\boldsymbol{a}$ and $\boldsymbol{b}$ into the last inequality in (25), the following inequality is obtained

$$
D^{2} e_{e m}[\delta J, \delta \boldsymbol{d} ; \delta J, \delta \boldsymbol{d}] \geq\left(2 \sqrt{2 g(J) g^{\prime \prime}(J)}-4\left|g^{\prime}(J)\right|\right)\left|g^{\prime}(J)\right||\delta J(\boldsymbol{d} \cdot \delta \boldsymbol{d})| .
$$

Hence, the convexity conditions in (20) follow from previous equation (26).

For the purely mechanical contribution $e_{m}(\boldsymbol{F})$, different models can be considered. A possible example includes the Mooney-Rivlin model $\left(e_{m}^{M R}\right)$, i.e.,

$$
e_{m}^{M R}(\boldsymbol{F})=\frac{\mu_{1}}{2} I I_{\boldsymbol{F}}+\frac{\mu_{2}}{2} I_{\boldsymbol{H}}+U^{M R}(J) ; \quad U^{M R}(J)=-\left(\mu_{1}+2 \mu_{2}\right) \ln J+\frac{\lambda}{2}(J-1)^{2},
$$

where $\left\{\mu_{1}, \mu_{2}, \lambda\right\}$ are material parameters, with units of stress, related to the shear modulus $\mu_{0}$ and the bulk modulus $\lambda_{0}$ in the origin as $\mu_{0}=\mu_{1}+\mu_{2}$ and $\lambda_{0}=\lambda+2 \mu_{2}$. Notice that the Mooney-Rivlin model $e_{m}^{M R}$ is convex with respect to $\{\boldsymbol{F}, \boldsymbol{H}, J\}$ (polyconvex).

\subsection{The Helmholtz energy function}

Alternatively, electromechanical constitutive models can be formulated in terms of a Helmholtz energy functional $\Psi\left(\boldsymbol{F}, \boldsymbol{E}_{0}\right)$, defined as

$$
\Psi\left(\boldsymbol{F}, \boldsymbol{E}_{0}\right)=-\sup _{\boldsymbol{D}_{0}}\left\{\boldsymbol{E}_{0} \cdot \boldsymbol{D}_{0}-e\left(\boldsymbol{F}, \boldsymbol{D}_{0}\right)\right\}
$$

As it is well known, it is possible to establish relationships between the first and second directional derivatives of above energy $\Psi\left(\boldsymbol{F}, \boldsymbol{E}_{0}\right)$ and those of $e\left(\boldsymbol{F}, \boldsymbol{D}_{0}\right)$. Indeed, the first directional derivative gives

$$
D \Psi\left[\delta \boldsymbol{F}, \delta \boldsymbol{E}_{0}\right]=\partial_{\boldsymbol{F}} \Psi: \delta \boldsymbol{F}+\partial_{\boldsymbol{E}_{0}} \Psi \cdot \delta \boldsymbol{E}_{0},
$$

with the partial derivatives of $\Psi$ and $e$ related as

$$
\partial_{\boldsymbol{F}} \Psi=\partial_{\boldsymbol{F}} e=\boldsymbol{P} ; \quad \partial_{\boldsymbol{E}_{0}} \Psi=-\boldsymbol{D}_{0} .
$$

As for the second directional derivative of $\Psi$, this can be formulated in terms of the Hessian $\left[\mathbb{H}_{\Psi}\right]$ in $\boldsymbol{F}, \boldsymbol{E}_{0}$ as

$$
D^{2} \Psi\left[\delta \boldsymbol{F}, \delta \boldsymbol{E}_{0} ; \delta \boldsymbol{F}, \delta \boldsymbol{E}_{0}\right]=\left[\delta \boldsymbol{F}: \delta \boldsymbol{E}_{0} \cdot\right]\left[\mathbb{H}_{\Psi}\right]\left[\begin{array}{l}
: \delta \boldsymbol{F} \\
\delta \boldsymbol{E}_{0}
\end{array}\right] ; \quad\left[\mathbb{H}_{\Psi}\right]=\left[\begin{array}{cc}
\mathcal{C} & -\mathcal{P}^{T} \\
-\mathcal{P} & -\boldsymbol{\epsilon}
\end{array}\right]
$$

where $\mathcal{C} \in \mathbb{R}^{3 \times 3 \times 3 \times 3}$ is an elasticity tensor; $\mathcal{P} \in \mathbb{R}^{3 \times 3 \times 3}$, the piezoelectric tensor, with $\left(\mathcal{P}^{T}\right)_{j J I}=$ $(\mathcal{P})_{I j J}$; and $\boldsymbol{\epsilon} \in \mathbb{R}^{3 \times 3}$, the dielectric tensor; and their expressions are given by

$$
\mathcal{C}\left(\boldsymbol{F}, \boldsymbol{E}_{0}\right)=\partial_{\boldsymbol{F} \boldsymbol{F}}^{2} \Psi ; \quad \mathcal{P}\left(\boldsymbol{F}, \boldsymbol{E}_{0}\right)=-\partial_{\boldsymbol{E}_{0} \boldsymbol{F}}^{2} \Psi ; \quad \boldsymbol{\epsilon}\left(\boldsymbol{F}, \boldsymbol{E}_{0}\right)=-\partial_{\boldsymbol{E}_{0} \boldsymbol{E}_{0}}^{2} \Psi
$$

It is possible [54] to relate the components of the Hessian operators of $\Psi$ and $e$ as

$$
\boldsymbol{\epsilon}=(\boldsymbol{\theta})^{-1} ; \quad \mathcal{P}^{T}=-\mathcal{Q} \cdot \boldsymbol{\epsilon} ; \quad \mathcal{C}=\mathcal{C}_{e}+\mathcal{Q}^{T} \cdot \mathcal{P}
$$


where the inner product - above indicates contraction of the indices placed immediately before and after. It is now interesting to compute the second directional derivative of the internal energy $e$ with respect to arguments $\boldsymbol{F}$ and $\boldsymbol{E}_{0}$ (instead of the natural $\boldsymbol{D}_{0}$ ). With that in mind, it is necessary to consider $\boldsymbol{D}_{0}$ expressed (implicitly) as a function of $\boldsymbol{F}$ and $\boldsymbol{E}_{0}$, namely $\boldsymbol{D}_{0}=$ $\tilde{\boldsymbol{D}}_{0}\left(\boldsymbol{F}, \boldsymbol{E}_{0}\right)$, to obtain

$$
\begin{aligned}
D \tilde{\boldsymbol{D}}_{0}\left[\delta \boldsymbol{F}, \delta \boldsymbol{E}_{0}\right] & =\partial_{\boldsymbol{F}} \tilde{\boldsymbol{D}}_{0}: \delta \boldsymbol{F}+\partial_{\boldsymbol{E}_{0}} \tilde{\boldsymbol{D}}_{0} \delta \boldsymbol{E}_{0} \\
& =-\partial_{\boldsymbol{E}_{0} \boldsymbol{F}}^{2} \Psi: \delta \boldsymbol{F}-\partial_{\boldsymbol{E}_{0} \boldsymbol{E}_{0}} \Psi \delta \boldsymbol{E}_{0} \\
& =\mathcal{P}: \delta \boldsymbol{F}+\boldsymbol{\epsilon} \delta \boldsymbol{E}_{0},
\end{aligned}
$$

where we have made use of equation (32) in the last line of above equation. Making now use of (33) and (34), we can express, after some algebraic manipulation, the second directional derivative of the internal energy $e$ with respect to $\boldsymbol{F}$ and $\boldsymbol{E}_{0}$ in terms of the second derivatives of the Helmholtz functional $\Psi\left(\boldsymbol{F}, \boldsymbol{E}_{0}\right)$ as

$$
D^{2} e\left[\delta \boldsymbol{F}, D \tilde{\boldsymbol{D}}_{0}\left[\delta \boldsymbol{F}, \delta \boldsymbol{E}_{0}\right] ; \delta \boldsymbol{F}, D \tilde{\boldsymbol{D}}_{0}\left[\delta \boldsymbol{F}, \delta \boldsymbol{E}_{0}\right]\right]=\delta \boldsymbol{F}: \mathcal{C}: \delta \boldsymbol{F}+\delta \boldsymbol{E}_{0} \cdot \boldsymbol{\epsilon} \delta \boldsymbol{E}_{0}
$$

As remarked in (9), to ensure existence of solutions in the vicinity of the origin, namely when $\boldsymbol{F} \approx \boldsymbol{I}$ and $\boldsymbol{E}_{0} \approx \mathbf{0}$, the internal energy functional must be strictly convex, i.e.,

$$
\left.D^{2} e\left[\delta \boldsymbol{F}, D \tilde{\boldsymbol{D}}_{0}\left[\delta \boldsymbol{F}, \delta \boldsymbol{E}_{0}\right] ; \delta \boldsymbol{F}, D \tilde{\boldsymbol{D}}_{0}\left[\delta \boldsymbol{F}, \delta \boldsymbol{E}_{0}\right]\right]\right|_{\boldsymbol{F}=\boldsymbol{I}, \boldsymbol{E}_{0}=\mathbf{0}}>0 ; \quad \forall \delta \boldsymbol{F}, \delta \boldsymbol{E}_{0},
$$

which requires positive definiteness of tensors $\mathcal{C}$ and $\boldsymbol{\epsilon}$, independently, that is, the Helmholtz energy functional $\Psi$ must be separately convex with respect to $\boldsymbol{F}$ and concave with respect to $\boldsymbol{E}_{0}$ (refer to (32)). The behaviour of both internal energy and Helmholtz functionals in the vicinity of the origin is illustrated in Figure 2. Unfortunately, away from the origin, it is very challenging to establish restrictions for the existence of solutions in terms of the Helmholtz free energy functional. That is the reason why it is preferable to define constitutive models in terms of the internal energy $e$, where, as it was shown in the previous section, the use of CMV functionals ensures the satisfaction of ellipticity in the most general case of electro-deformation. Indeed, even when the internal energy functional $e\left(\boldsymbol{F}, \boldsymbol{D}_{0}\right)$ complies with the multi-variable convexity condition in (13), the dual Helmholtz functional $\Psi\left(\boldsymbol{F}, \boldsymbol{E}_{0}\right)$, which will be concave with respect to $\boldsymbol{E}_{0}$, can potentially lose its polyconvexity with respect to $\boldsymbol{F}$.

Finally, notice that the Helmholtz free energy $\Psi$ and its first and second directional derivatives can still be accessed via equations (28), (30) and (33), which is extremely useful in the case of using variational principles based on displacements and electric potential, as it is the case of this paper. Furthermore, in the case of using CMV energy functionals, the internal energy $W(\mathcal{V})$ and its first and second directional derivatives can be related to those of $e$ as presented in [31, 53].

\section{Constitutive equations in nonlinear electro-elasticity: Application to Rank-One layered EAPs}

\subsection{Effective or macroscopic internal energy functional}

In this paper we study the homogenised behaviour of rank-one layered EAPs. The resulting biphasic layered structure is comprised of constituents or phases $a$ and $b$ with volume fractions $c^{a}=h_{0}^{a} /\left(h_{0}^{a}+h_{0}^{b}\right)$ and $c^{b}=1-c^{a}$, respectively, where $h_{0}^{a}$ and $h_{0}^{b}$ denote the thickness of phases $a$ and $b$ in the undeformed configuration $\mathcal{B}_{0}$, with $h_{0}^{a}, h_{0}^{b}<<1$ to comply with homogenisation theory. The interface between both phases is characterised by the normal vector $\boldsymbol{N}^{8}$, spherically parametrised as $\boldsymbol{N}=\left[\begin{array}{lll}\sin \bar{\beta} \cos \bar{\alpha}, & \sin \bar{\beta} \sin \bar{\alpha}, \quad \cos \bar{\beta}\end{array}\right]^{T}$. A scheme illustrating the structure of the rank-one layered composite is shown in Figure 3.

\footnotetext{
${ }^{8}$ Throughout this Section, this unit normal is not to be confused with the outward unit normal to the boundary presented in Section 2.
} 

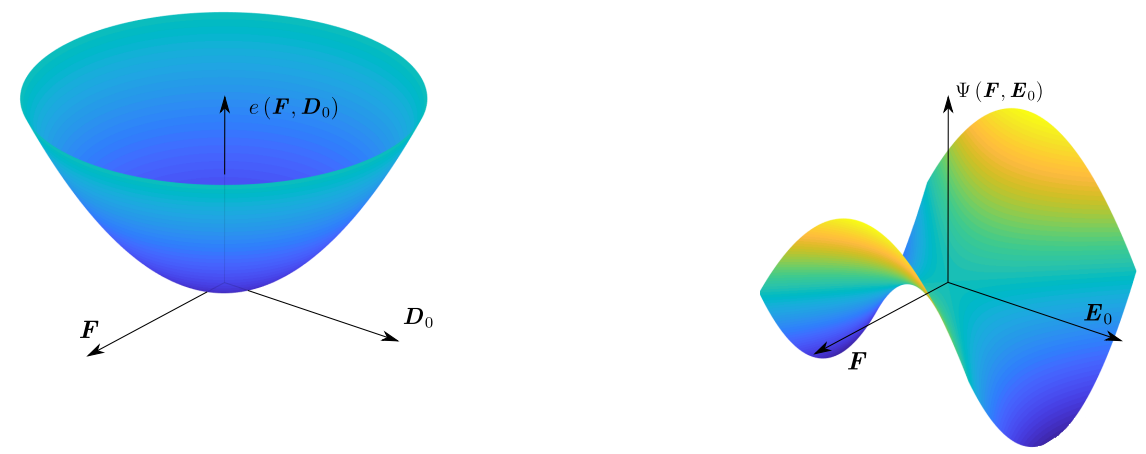

Figure 2: Convexity of the internal energy functional $e\left(\boldsymbol{F}, \boldsymbol{D}_{0}\right)$ in the vicinity of the origin. Simultaneous convexity and concavity (saddle point nature) of the (dual) Helmholtz functional $\Psi\left(\boldsymbol{F}, \boldsymbol{E}_{0}\right)$ in the origin with respect to $\boldsymbol{F}$ and $\boldsymbol{E}_{0}$, respectively.

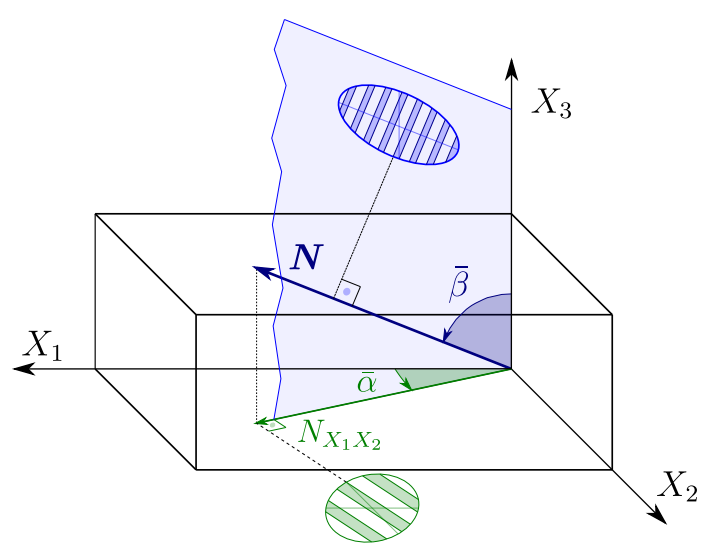

(a)

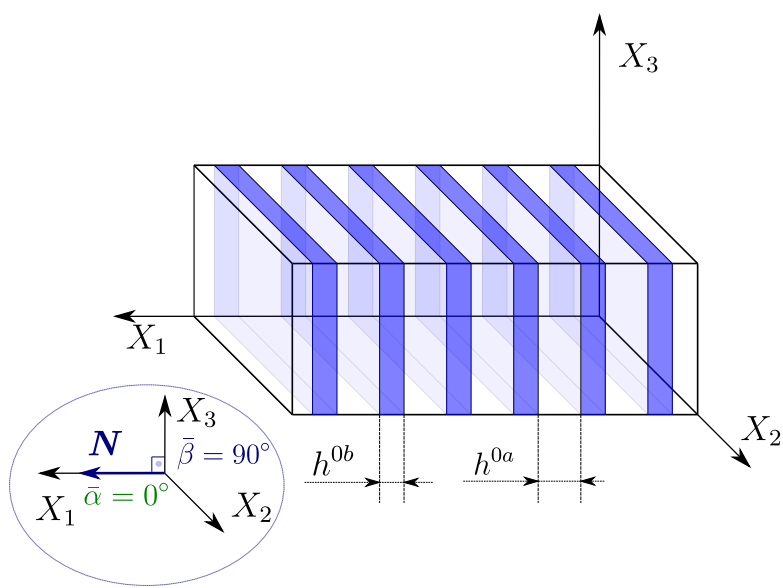

(b)

Figure 3: Description of a 3-D rank-one laminated composite material: (a) general case, (b) particularisation for $\bar{\alpha}=0^{\circ}, \bar{\beta}=0^{\circ}$, for visualisation purposes.

Under the assumption of a homogeneous response in each phase, the macroscopic deformation gradient $\boldsymbol{F}$ and the macroscopic electric displacement field $\boldsymbol{D}_{0}$ are defined as the weighted sum of those in each phase, namely

$$
\boldsymbol{F}=c^{a} \boldsymbol{F}^{a}+c^{b} \boldsymbol{F}^{b} ; \quad \boldsymbol{D}_{0}=c^{a} \boldsymbol{D}_{0}^{a}+c^{b} \boldsymbol{D}_{0}^{b},
$$

where the upper indices $a$ and $b$ are used to indicate the respective microscale phases. In addition, we enforce strong continuity of the tangential and normal components of the deformation gradient and the electric displacement, respectively, across the laminate interface, that is,

$$
\llbracket \boldsymbol{F} \rrbracket \times N=\mathbf{0} ; \quad \llbracket \boldsymbol{D}_{0} \rrbracket \cdot \boldsymbol{N}=0,
$$

where $\llbracket \bullet \rrbracket$ denotes the standard jump operator across the interface, defined as $\llbracket \bullet \rrbracket=(\bullet)^{a}-(\bullet)^{b}$. Above jump conditions (38) can alternatively be written in a more convenient form as

$$
\llbracket \boldsymbol{F} \rrbracket=\boldsymbol{\alpha} \otimes N ; \quad \llbracket \boldsymbol{D}_{0} \rrbracket=\mathcal{T}_{\boldsymbol{N}} \boldsymbol{\beta},
$$

where $\boldsymbol{\alpha} \in \mathbb{R}^{3}$ denotes the microscale deformation gradient amplitude vector; $\boldsymbol{\beta} \in \mathbb{R}^{2}$, the microscale electric displacement amplitude vector; and $\mathcal{T}_{\boldsymbol{N}} \in \mathbb{R}^{3 \times 2}$, a projection operator onto the plane normal to the unit vector $\boldsymbol{N}$ defined as $\boldsymbol{T}_{\boldsymbol{N}}=\boldsymbol{T}_{1} \otimes \boldsymbol{E}_{1}+\boldsymbol{T}_{2} \otimes \boldsymbol{E}_{2}$, where $\boldsymbol{T}_{1}$ and $\boldsymbol{T}_{2}$ are two linearly independent unit vectors contained within the laminate plane, and $\boldsymbol{E}_{1}=\left[\begin{array}{ll}1 & 0\end{array}\right]^{T}$ and $\boldsymbol{E}_{2}=\left[\begin{array}{ll}0 & 1\end{array}\right]^{T}$. 
Combination of equations (37) and (39) permits to obtain the microscale deformation gradient $\boldsymbol{F}^{a, b}$ and electric displacement $\boldsymbol{D}_{0}^{a, b}$ as

$$
\begin{array}{lll}
\boldsymbol{F}^{a}(\boldsymbol{F}, \boldsymbol{\alpha})=\boldsymbol{F}+c^{b} \boldsymbol{\alpha} \otimes \boldsymbol{N} ; & \boldsymbol{D}_{0}^{a}\left(\boldsymbol{D}_{0}, \boldsymbol{\beta}\right)=\boldsymbol{D}_{0}+c^{b} \mathcal{T}_{\boldsymbol{N}} \boldsymbol{\beta} \\
\boldsymbol{F}^{b}(\boldsymbol{F}, \boldsymbol{\alpha})=\boldsymbol{F}-c^{a} \boldsymbol{\alpha} \otimes \boldsymbol{N} ; & \boldsymbol{D}_{0}^{b}\left(\boldsymbol{D}_{0}, \boldsymbol{\beta}\right)=\boldsymbol{D}_{0}-c^{a} \mathcal{T}_{\boldsymbol{N}} \boldsymbol{\beta},
\end{array}
$$

from where their first directional derivatives can be computed as

$$
\begin{aligned}
D \boldsymbol{F}^{a}[\delta \boldsymbol{F}] & =\delta \boldsymbol{F} ; & D \boldsymbol{F}^{a}[\delta \boldsymbol{\alpha}] & =c^{b} \delta \boldsymbol{\alpha} \otimes \boldsymbol{N} ; \\
D \boldsymbol{D}_{0}^{a}\left[\delta \boldsymbol{D}_{0}\right] & =\delta \boldsymbol{D}_{0} ; & D \boldsymbol{D}_{0}^{a}[\delta \boldsymbol{\beta}] & =c^{b} \mathcal{T}_{\boldsymbol{N}} \delta \boldsymbol{\beta},
\end{aligned}
$$

for phase $a$, and similarly for phase $b$ as

$$
\begin{aligned}
& D \boldsymbol{F}^{b}[\delta \boldsymbol{F}]=\delta \boldsymbol{F} ; \quad D \boldsymbol{F}^{b}[\delta \boldsymbol{\alpha}]=-c^{a} \delta \boldsymbol{\alpha} \otimes \boldsymbol{N} ; \\
& D \boldsymbol{D}_{0}^{b}\left[\delta \boldsymbol{D}_{0}\right]=\delta \boldsymbol{D}_{0} ; \quad D \boldsymbol{D}_{0}^{b}[\delta \boldsymbol{\beta}]=-c^{a} \mathcal{T}_{\boldsymbol{N}} \delta \boldsymbol{\beta} .
\end{aligned}
$$

It is customary to postulate the homogenised or effective internal energy functional of the composite $e\left(\boldsymbol{F}, \boldsymbol{D}_{0}\right)$ as

$$
e\left(\boldsymbol{F}, \boldsymbol{D}_{0}\right)=\min _{\boldsymbol{\alpha}, \boldsymbol{\beta}}\left\{\hat{e}\left(\boldsymbol{F}, \boldsymbol{D}_{0}, \boldsymbol{\alpha}, \boldsymbol{\beta}\right)\right\}
$$

with

$$
\hat{e}\left(\boldsymbol{F}, \boldsymbol{D}_{0}, \boldsymbol{\alpha}, \boldsymbol{\beta}\right)=c^{a} e^{a}\left(\boldsymbol{F}^{a}(\boldsymbol{F}, \boldsymbol{\alpha}), \boldsymbol{D}_{0}^{a}\left(\boldsymbol{D}_{0}, \boldsymbol{\beta}\right)\right)+c^{b} e^{b}\left(\boldsymbol{F}^{b}(\boldsymbol{F}, \boldsymbol{\alpha}), \boldsymbol{D}_{0}^{b}\left(\boldsymbol{D}_{0}, \boldsymbol{\beta}\right)\right),
$$

where $e^{a}=e^{a}\left(\boldsymbol{F}^{a}, \boldsymbol{D}_{0}^{a}\right)$ and $e^{b}=e^{b}\left(\boldsymbol{F}^{b}, \boldsymbol{D}_{0}^{b}\right)$ are the microscale internal energy functions expressed in terms of their respective microscale fields. The stationary conditions of (44) with respect to the microscale amplitude vectors are defined as

$$
D \hat{e}[\delta \boldsymbol{\alpha}, \delta \boldsymbol{\beta}]=D \hat{e}[\delta \boldsymbol{\alpha}]+D \hat{e}[\delta \boldsymbol{\beta}]=0 ; \quad \forall \delta \boldsymbol{\alpha}, \delta \boldsymbol{\beta},
$$

where

$$
\begin{aligned}
& D \hat{e}[\delta \boldsymbol{\alpha}]=c^{a} \partial_{\boldsymbol{F}^{a}} e^{a}: D \boldsymbol{F}^{a}[\delta \boldsymbol{\alpha}]+c^{b} \partial_{\boldsymbol{F}^{b}} e^{b}: D \boldsymbol{F}^{b}[\delta \boldsymbol{\alpha}] \\
& =c^{a} c^{b}\left(\partial_{\boldsymbol{F}^{a}} e^{a}-\partial_{\boldsymbol{F}^{b}} e^{b}\right):(\delta \boldsymbol{\alpha} \otimes \boldsymbol{N}) \\
& =c^{a} c^{b}\left(\boldsymbol{P}^{a}-\boldsymbol{P}^{b}\right):(\delta \boldsymbol{\alpha} \otimes \boldsymbol{N}) \text {; } \\
& D \hat{e}[\delta \boldsymbol{\beta}]=c^{a} \partial_{\boldsymbol{D}_{0}^{a}} e^{a} \cdot D \boldsymbol{D}_{0}^{a}[\delta \boldsymbol{\alpha}]+c^{b} \partial_{\boldsymbol{D}_{0}^{b}} e^{b} \cdot D \boldsymbol{D}_{0}^{b}[\delta \boldsymbol{\beta}] \\
& =c^{a} c^{b}\left(\partial_{\boldsymbol{D}_{0}^{a}} e^{a}-\partial_{\boldsymbol{D}_{0}^{b}} e^{b}\right) \cdot\left(\boldsymbol{\mathcal { T }}_{\boldsymbol{N}} \delta \boldsymbol{\beta}\right) \\
& =c^{a} c^{b}\left(\boldsymbol{E}_{0}^{a}-\boldsymbol{E}_{0}^{b}\right) \cdot\left(\mathcal{T}_{\boldsymbol{N}} \delta \boldsymbol{\beta}\right) \text {. }
\end{aligned}
$$

In above equations (46a) and (46b), we have made use of the directional derivatives in (41) and (42) and the constitutive relationships (6) for each phase $a$ and $b$. Considering now independent variations $\delta \boldsymbol{\alpha}$ and $\delta \boldsymbol{\beta}$, the stationary conditions (45) become

$$
\llbracket P \rrbracket \boldsymbol{N}=\mathbf{0} ; \quad \boldsymbol{\mathcal { T }}_{\boldsymbol{N}}^{T} \llbracket \boldsymbol{E}_{0} \rrbracket=\mathbf{0} \Longleftrightarrow \llbracket \boldsymbol{E}_{0} \rrbracket \times \boldsymbol{N}=\mathbf{0},
$$

which represent the strong enforcement of the normal and tangential components of the traction vector and the electric field vector, respectively. Thus, above homogenised energy functional $e\left(\boldsymbol{F}, \boldsymbol{D}_{0}\right)$ can be re-written as

$$
e\left(\boldsymbol{F}, \boldsymbol{D}_{0}\right)=\left.\hat{e}\left(\boldsymbol{F}, \boldsymbol{D}_{0}, \boldsymbol{\alpha}, \boldsymbol{\beta}\right)\right|_{\text {s.t. }\left\{\llbracket \boldsymbol{P} \rrbracket \boldsymbol{N}=\mathbf{0} ; \llbracket \boldsymbol{E}_{0} \rrbracket \times \boldsymbol{N}=\mathbf{0}\right\}} .
$$


Remark 4. The expression for the microscopic deformation gradient tensors $\left\{\boldsymbol{F}^{a}, \boldsymbol{F}^{b}\right\}$ in terms of the macroscopic deformation gradient tensor $\boldsymbol{F}$ and the spatial amplitude vector $\boldsymbol{\alpha}$ in equation (40) follows an unconventional additive decomposition. Although this decomposition was shown first in [19] in the mechanical context, a multiplicative decomposition [5, 19] has been traditionally preferred, whereby $\left\{\boldsymbol{F}^{a}, \boldsymbol{F}^{b}\right\}$ are defined in terms of a material amplitude vector $\widetilde{\boldsymbol{\alpha}}$ as

$$
\boldsymbol{F}^{a}=\boldsymbol{F}\left(\boldsymbol{I}+c^{b} \widetilde{\boldsymbol{\alpha}} \otimes \boldsymbol{N}\right) ; \quad \boldsymbol{F}^{b}=\boldsymbol{F}\left(\boldsymbol{I}-c^{a} \widetilde{\boldsymbol{\alpha}} \otimes \boldsymbol{N}\right) .
$$

Notice that both additive and multiplicative decompositions are equivalent. Indeed, comparison of equations (49) and (40) enables to establish a relationship between the material amplitude vector $\widetilde{\boldsymbol{\alpha}}$ in (49) and its Eulerian or spatial counterpart $\boldsymbol{\alpha}$, associated with the additive decomposition, in (40) as

$$
\boldsymbol{\alpha}=\boldsymbol{F} \widetilde{\boldsymbol{\alpha}} .
$$

However, the choice of the additive decomposition followed in this paper is not motivated by a pure formalism. On the contrary, the lower degree of nonlinearity associated with this additive decomposition entails a considerable simplification from the algebraic standpoint. Specifically, this reflects in a higher tractability of the second directional derivatives of the effective internal energy functional $e\left(\boldsymbol{F}, \boldsymbol{D}_{0}\right)$, and hence of the effective constitutive tensors associated with it, namely $\left\{\mathcal{C}_{e}, \mathcal{Q}, \boldsymbol{\theta}\right\}$

\subsection{Solution of the amplitude vectors $\boldsymbol{\alpha}$ and $\boldsymbol{\beta}$}

The stationary conditions (45), or their equivalent jump conditions (47), represent a system of nonlinear equations where the microscale amplitude vectors $\{\boldsymbol{\alpha}, \boldsymbol{\beta}\}$ can be resolved in terms of the macroscale homogenised fields $\boldsymbol{F}, \boldsymbol{D}_{0}$. To ensure existence of solutions, the second directional derivative of $\hat{e}$ with respect to the microscale amplitude vectors must be (strictly) convex, namely

$$
D^{2} \hat{e}[\delta \boldsymbol{\alpha}, \delta \boldsymbol{\beta} ; \delta \boldsymbol{\alpha}, \delta \boldsymbol{\beta}]>0 ; \quad \forall \delta \boldsymbol{\alpha}, \delta \boldsymbol{\beta},
$$

where

$$
D^{2} \hat{e}[\delta \boldsymbol{\alpha}, \delta \boldsymbol{\beta} ; \delta \boldsymbol{\alpha}, \delta \boldsymbol{\beta}]=D^{2} \hat{e}[\delta \boldsymbol{\alpha} ; \delta \boldsymbol{\alpha}]+D^{2} \hat{e}[\delta \boldsymbol{\alpha} ; \delta \boldsymbol{\beta}]+D^{2} \hat{e}[\delta \boldsymbol{\beta} ; \delta \boldsymbol{\alpha}]+D^{2} \hat{e}[\delta \boldsymbol{\beta} ; \delta \boldsymbol{\beta}],
$$

and each of the terms on the right-hand side of above equation (52) can be found as

$$
\begin{aligned}
& D^{2} \hat{e}[\delta \boldsymbol{\alpha} ; \delta \boldsymbol{\alpha}]=(\delta \boldsymbol{\alpha} \otimes \boldsymbol{N}): c^{a} c^{b}\left[\partial_{\boldsymbol{F}^{a} \boldsymbol{F}^{a}}^{2} e^{a}: D \boldsymbol{F}^{a}[\delta \boldsymbol{\alpha}]-\partial_{\boldsymbol{F}^{b} \boldsymbol{F}^{b}}^{2} e^{b}: D \boldsymbol{F}^{b}[\delta \boldsymbol{\alpha}]\right] \\
& =(\delta \boldsymbol{\alpha} \otimes \boldsymbol{N}): c^{a} c^{b}\left[c^{b} \partial_{\boldsymbol{F}^{a}}^{2} \boldsymbol{F}^{a} e^{a}+c^{a} \partial_{\boldsymbol{F}^{b} \boldsymbol{F}^{b}} e^{b}\right]:(\delta \boldsymbol{\alpha} \otimes \boldsymbol{N}) \\
& =(\delta \boldsymbol{\alpha} \otimes \boldsymbol{N}): c^{a} c^{b}\left[c^{b} \mathcal{C}_{e}^{a}+c^{a} \mathcal{C}_{e}^{b}\right]:(\delta \boldsymbol{\alpha} \otimes \boldsymbol{N}) \\
& D^{2} \hat{e}[\delta \boldsymbol{\beta} ; \delta \boldsymbol{\alpha}]=\left(\boldsymbol{T}_{\boldsymbol{N}} \delta \boldsymbol{\beta}\right) \cdot c^{a} c^{b}\left[\partial_{\boldsymbol{D}_{0}^{a} \boldsymbol{F}^{a}}^{2} e^{a}: D \boldsymbol{F}^{a}[\delta \boldsymbol{\alpha}]-\partial_{\boldsymbol{D}_{0}^{b} \boldsymbol{F}^{b}}^{2} e^{b}: D \boldsymbol{F}^{b}[\delta \boldsymbol{\alpha}]\right] \\
& =\left(\boldsymbol{T}_{\boldsymbol{N}} \delta \boldsymbol{\beta}\right) \cdot c^{a} c^{b}\left[c^{b} \partial_{\boldsymbol{D}_{0}^{a} \boldsymbol{F}^{a}}^{2} e^{a}+c^{a} \partial_{\boldsymbol{D}_{0}^{b} \boldsymbol{F}^{b}}^{b}\right]:(\delta \boldsymbol{\alpha} \otimes \boldsymbol{N}) \\
& =\left(\boldsymbol{T}_{\boldsymbol{N}} \delta \boldsymbol{\beta}\right) \cdot c^{a} c^{b}\left[c^{b} \mathcal{Q}^{a}+c^{a} \mathcal{Q}^{b}\right]:(\delta \boldsymbol{\alpha} \otimes \boldsymbol{N})=D^{2} \hat{e}[\delta \boldsymbol{\alpha} ; \delta \boldsymbol{\beta}] ;
\end{aligned}
$$

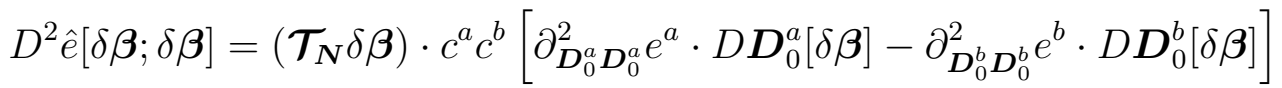

$$
\begin{aligned}
& =\left(\boldsymbol{\mathcal { T }}_{\boldsymbol{N}} \delta \boldsymbol{\beta}\right) \cdot c^{a} c^{b}\left[c^{b} \partial_{\boldsymbol{D}_{0}^{a} \boldsymbol{D}_{0}^{a}}^{2} e^{a}+c^{a} \partial_{\boldsymbol{D}_{0}^{b} \boldsymbol{D}_{0}^{b}}^{2} e^{b}\right]\left(\boldsymbol{\mathcal { T }}_{\boldsymbol{N}} \delta \boldsymbol{\beta}\right) \\
& =\left(\boldsymbol{\mathcal { T }}_{\boldsymbol{N}} \delta \boldsymbol{\beta}\right) \cdot c^{a} c^{b}\left[c^{b} \boldsymbol{\theta}^{a}+c^{a} \boldsymbol{\theta}^{b}\right]\left(\mathcal{T}_{\boldsymbol{N}} \delta \boldsymbol{\beta}\right) .
\end{aligned}
$$


In above equations (53a), (53b) and (53c), we have made repetitive use of the directional derivatives (46a) and (46b) and the constitutive relationships (8). Substitution of above equations (53a), (53b) and (53c) into (52), and grouping, yields

$$
D^{2} \hat{e}[\delta \boldsymbol{\alpha}, \delta \boldsymbol{\beta} ; \delta \boldsymbol{\alpha}, \delta \boldsymbol{\beta}]=\left[\delta \boldsymbol{\alpha} \otimes \boldsymbol{N}: \quad \mathcal{T}_{\boldsymbol{N}} \delta \boldsymbol{\beta} \cdot\right] c^{a} c^{b}\left[\hat{\mathbb{H}}_{e}\right]\left[\begin{array}{c}
: \delta \boldsymbol{\alpha} \otimes \boldsymbol{N} \\
\mathcal{T}_{\boldsymbol{N}} \delta \boldsymbol{\beta}
\end{array}\right]
$$

where $\left[\hat{\mathbb{H}}_{e}\right]$ represents an average Hessian operator of the microscale constituents, defined as

$$
\left[\hat{\mathbb{H}}_{e}\right]=c^{b}\left[\mathbb{H}_{e}^{a}\right]+c^{a}\left[\mathbb{H}_{e}^{b}\right] ; \quad\left[\hat{\mathbb{H}}_{e}\right]=\left[\begin{array}{cc}
\hat{\mathcal{C}}_{e} & \hat{\mathcal{Q}}^{T} \\
\hat{\mathcal{Q}} & \hat{\boldsymbol{\theta}}
\end{array}\right] ; \quad\left[\mathbb{H}_{e}^{a, b}\right]=\left[\begin{array}{cc}
\mathcal{C}_{e}^{a, b} & \mathcal{\mathcal { Q }}^{a, b, T} \\
\mathcal{\mathcal { Q }}^{a, b} & \boldsymbol{\theta}^{a, b}
\end{array}\right]
$$

where $\left[\mathbb{H}_{e}^{a, b}\right]$ represent the Hessian operators of the microscale internal energy contributions with respect to their own fields $\left\{\boldsymbol{F}^{a, b}, \boldsymbol{D}_{0}^{a, b}\right\}$. Notice that an alternative representation to equation (54) can be formulated in terms of the reduced Hessian of the internal energy $\hat{e}$ with respect to the microscale amplitude vectors, that is,

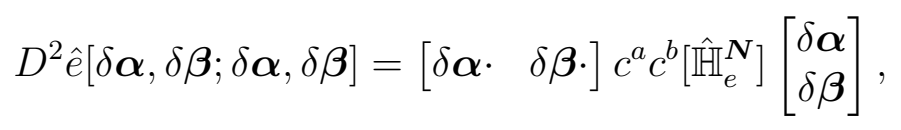

where

$$
\left[\hat{\mathbb{H}}_{e}^{\boldsymbol{N}}\right]=\left[\begin{array}{cc}
\hat{\mathcal{C}}_{e, \boldsymbol{N} \boldsymbol{N}} & \boldsymbol{N} \cdot \hat{\mathcal{Q}}^{T} \mathcal{T}_{\boldsymbol{N}} \\
\mathcal{T}_{\boldsymbol{N}}^{T} \hat{\mathcal{Q}} \boldsymbol{N} & \mathcal{T}_{\boldsymbol{N}}^{T} \hat{\boldsymbol{\theta}} \mathcal{T}_{\boldsymbol{N}}
\end{array}\right]
$$

where $\left(\hat{\boldsymbol{C}}_{e, N \boldsymbol{N}}\right)_{i j}=\left(\hat{\boldsymbol{C}}_{e}\right)_{i I j J} N_{I} N_{J}$. It remains to demonstrate that convexity condition (51) is fulfilled or, alternatively, the positive definiteness of the reduced Hessian operator $\left[\hat{\mathbb{H}}_{e}^{N}\right]$. This is straightforward to notice by re-writing (54) as

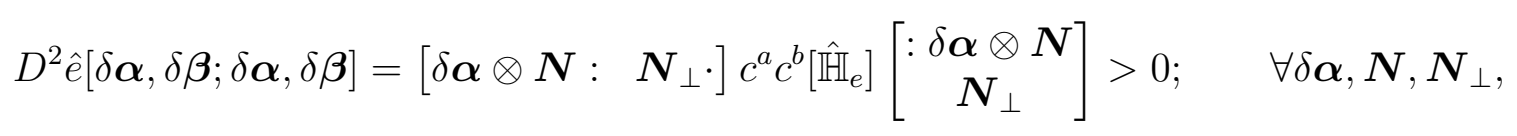

where $\mathcal{T}_{\boldsymbol{N}} \delta \boldsymbol{\beta}$ has been renamed as $\boldsymbol{N}_{\perp}$ by noticing that it represents a vector contained within the laminate plane. As it is immediate to see, the right-hand side of above equation (58) is (strictly) positive provided that microscale internal energy functionals $e^{a}$ and $e^{b}$ are individually elliptic (refer to equation (10)). Thus, provided that each of the phases is defined via CMV functionals, above inequality (58) is fulfilled, and thus existence of solution $\boldsymbol{\alpha}, \boldsymbol{\beta}$ is always guaranteed.

Once the existence of solution $\boldsymbol{\alpha}, \boldsymbol{\beta}$ is shown, its computation can be carried out via a $k$ iterative Newton-Raphson algorithm, namely

$$
\left.D \hat{e}[\delta \boldsymbol{\alpha}, \delta \boldsymbol{\beta}]\right|^{k}+\left.D^{2} \hat{e}[\delta \boldsymbol{\alpha}, \delta \boldsymbol{\beta} ; \Delta \boldsymbol{\alpha}, \Delta \boldsymbol{\beta}]\right|^{k}=0 ; \quad \boldsymbol{\alpha}^{k+1}=\boldsymbol{\alpha}^{k}+\Delta \boldsymbol{\alpha} ; \quad \boldsymbol{\beta}^{k+1}=\boldsymbol{\beta}^{k}+\Delta \boldsymbol{\beta},
$$

which can alternatively be written as

$$
\left[\begin{array}{c}
\Delta \boldsymbol{\alpha} \\
\Delta \boldsymbol{\beta}
\end{array}\right]=-\left[\hat{\mathbb{H}}_{e}^{\boldsymbol{N}}\right]_{k}^{-1}\left[\begin{array}{c}
\llbracket \boldsymbol{P} \rrbracket \boldsymbol{N} \\
\mathcal{T}_{\boldsymbol{N}}^{T} \llbracket \boldsymbol{E}_{0} \rrbracket
\end{array}\right]_{k}
$$

which involves an iterative scheme where microscale amplitude vectors are iterated until convergence (strong satisfaction of jump conditions) is fulfilled. 


\subsection{Effective stress and electric field}

Solution of the microscale amplitude vectors $\{\boldsymbol{\alpha}, \boldsymbol{\beta}\}$ in terms of the macroscale deformation gradient and electric displacement $\left\{\boldsymbol{F}, \boldsymbol{D}_{0}\right\}$ permits to express the microscale deformation gradient and electric displacement of the phases as

$$
\begin{array}{rlrl}
\tilde{\boldsymbol{F}}^{a}\left(\boldsymbol{F}, \boldsymbol{D}_{0}\right) & =\boldsymbol{F}+c^{b} \tilde{\boldsymbol{\alpha}}\left(\boldsymbol{F}, \boldsymbol{D}_{0}\right) \otimes \boldsymbol{N} ; & & \tilde{\boldsymbol{D}}_{0}^{a}\left(\boldsymbol{F}, \boldsymbol{D}_{0}\right)=\boldsymbol{D}_{0}+c^{b} \mathcal{T}_{\boldsymbol{N}} \tilde{\boldsymbol{\beta}}\left(\boldsymbol{F}, \boldsymbol{D}_{0}\right) \\
\tilde{\boldsymbol{F}}^{b}\left(\boldsymbol{F}, \boldsymbol{D}_{0}\right)=\boldsymbol{F}-c^{a} \tilde{\boldsymbol{\alpha}}\left(\boldsymbol{F}, \boldsymbol{D}_{0}\right) \otimes \boldsymbol{N} ; & & \tilde{\boldsymbol{D}}_{0}^{b}\left(\boldsymbol{F}, \boldsymbol{D}_{0}\right)=\boldsymbol{D}_{0}-c^{a} \mathcal{T}_{\boldsymbol{N}} \tilde{\boldsymbol{\beta}}\left(\boldsymbol{F}, \boldsymbol{D}_{0}\right),
\end{array}
$$

where the $\operatorname{sign}(\tilde{\bullet})$ is used to indicate the implicit dependence of the amplitude vectors in terms of the macroscale fields. As a result, the directional derivatives for phase $a$ are

$$
\begin{aligned}
& D \tilde{\boldsymbol{F}}^{a}[\delta \boldsymbol{F}]=\delta \boldsymbol{F}+c^{b} D \tilde{\boldsymbol{\alpha}}[\delta \boldsymbol{F}] \otimes \boldsymbol{N} ; \quad D \tilde{\boldsymbol{D}}_{0}^{a}[\delta \boldsymbol{F}]=c^{b} \mathcal{T}_{\boldsymbol{N}} D \tilde{\boldsymbol{\beta}}[\delta \boldsymbol{F}] ; \\
& D \tilde{\boldsymbol{F}}^{a}\left[\delta \boldsymbol{D}_{0}\right]=c^{b} D \tilde{\boldsymbol{\alpha}}\left[\delta \boldsymbol{D}_{0}\right] \otimes \boldsymbol{N} ; \quad D \tilde{\boldsymbol{D}}_{0}^{a}\left[\delta \boldsymbol{D}_{0}\right]=\delta \boldsymbol{D}_{0}+c^{b} \boldsymbol{T}_{\boldsymbol{N}} D \tilde{\boldsymbol{\beta}}\left[\delta \boldsymbol{D}_{0}\right],
\end{aligned}
$$

and, analogously, for phase $b$ are

$$
\begin{aligned}
D \tilde{\boldsymbol{F}}^{b}[\delta \boldsymbol{F}] & =\delta \boldsymbol{F}-c^{a} D \tilde{\boldsymbol{\alpha}}[\delta \boldsymbol{F}] \otimes \boldsymbol{N} ; & D \tilde{\boldsymbol{D}}_{0}^{b}[\delta \boldsymbol{F}] & =-c^{a} \mathcal{T}_{\boldsymbol{N}} D \tilde{\boldsymbol{\beta}}[\delta \boldsymbol{F}] ; \\
D \tilde{\boldsymbol{F}}^{b}\left[\delta \boldsymbol{D}_{0}\right] & =-c^{a} D \tilde{\boldsymbol{\alpha}}\left[\delta \boldsymbol{D}_{0}\right] \otimes \boldsymbol{N} ; & D \tilde{\boldsymbol{D}}_{0}^{b}\left[\delta \boldsymbol{D}_{0}\right] & =\delta \boldsymbol{D}_{0}-c^{a} \boldsymbol{\mathcal { T }}_{\boldsymbol{N}} D \tilde{\boldsymbol{\beta}}\left[\delta \boldsymbol{D}_{0}\right]
\end{aligned}
$$

Making use of expressions in (62a), (63a), the directional derivative of the effective energy $e\left(\boldsymbol{F}, \boldsymbol{D}_{0}\right)$ in $(43)$ with respect to the macroscopic deformation gradient tensor $\boldsymbol{F}$ yields

$$
\begin{aligned}
D e[\delta \boldsymbol{F}] & =c^{a} \partial_{\boldsymbol{F}^{a}} e^{a}: \underbrace{\left(\delta \boldsymbol{F}+c^{b} D \tilde{\boldsymbol{\alpha}}[\delta \boldsymbol{F}] \otimes \boldsymbol{N}\right)}_{D \tilde{\boldsymbol{F}}^{a}[\delta \boldsymbol{F}]}+c^{b} \partial_{\boldsymbol{F}^{b} e^{b}: \underbrace{\left(\delta \boldsymbol{F}-c^{a} D \tilde{\boldsymbol{\alpha}}[\delta \boldsymbol{F}] \otimes \boldsymbol{N}\right)}_{D \tilde{\boldsymbol{F}}^{b}[\delta \boldsymbol{F}]}} \\
& +c^{a} \partial_{\boldsymbol{D}_{0}^{a}} e^{a} \cdot \underbrace{\left(c^{b} \boldsymbol{\mathcal { T }}_{\boldsymbol{N}} D \tilde{\boldsymbol{\beta}}[\delta \boldsymbol{F}]\right)}_{D \tilde{\boldsymbol{D}}_{0}^{a}[\delta \boldsymbol{F}]}+c^{b} \partial_{\boldsymbol{D}_{0}^{b}} e^{b} \cdot \underbrace{\left(-c^{a} \boldsymbol{\mathcal { T }}_{\boldsymbol{N}} D \tilde{\boldsymbol{\beta}}[\delta \boldsymbol{F}]\right)}_{D \tilde{\boldsymbol{D}}_{0}^{b}[\delta \boldsymbol{F}]} .
\end{aligned}
$$

Suitable manipulation of above equation (64) permits to arrive at the following expression

$$
D e[\delta \boldsymbol{F}]=\left(c^{a} \boldsymbol{P}^{a}+c^{b} \boldsymbol{P}^{b}\right): \delta \boldsymbol{F}+\underbrace{c^{a} c^{b} D \tilde{\boldsymbol{\alpha}}[\delta \boldsymbol{F}] \cdot(\llbracket \boldsymbol{P} \rrbracket \boldsymbol{N})}_{=0}+\underbrace{c^{a} c^{b} D \tilde{\boldsymbol{\beta}}[\delta \boldsymbol{F}] \cdot\left(\mathcal{T}_{\boldsymbol{N}}^{T} \llbracket \boldsymbol{E}_{0} \rrbracket\right)}_{=0},
$$

where use of the stationary conditions in (47) enables to cancel the last two contributions in (65). Similarly, making use of expressions in (62b), (63b), the directional derivative of the effective energy $e\left(\boldsymbol{F}, \boldsymbol{D}_{0}\right)$ in (43) with respect to the macroscopic electric displacement $\boldsymbol{D}_{0}$ yields

$$
\begin{aligned}
D e\left[\delta \boldsymbol{D}_{0}\right] & =c^{a} \partial_{\boldsymbol{F}^{a}} e^{a}: \underbrace{\left(c^{b} D \tilde{\boldsymbol{\alpha}}\left[\delta \boldsymbol{D}_{0}\right] \otimes \boldsymbol{N}\right)}_{D \tilde{\boldsymbol{F}}^{a}\left[\delta \boldsymbol{D}_{0}\right]}+c^{b} \partial_{\boldsymbol{F}^{b}} e^{b}: \underbrace{\left(-c^{a} D \tilde{\boldsymbol{\beta}}\left[\delta \boldsymbol{D}_{0}\right] \otimes \boldsymbol{N}\right)}_{D \tilde{\boldsymbol{F}}^{b}\left[\delta \boldsymbol{D}_{0}\right]} \\
& +c^{a} \partial_{\boldsymbol{D}_{0}^{a}} e^{a} \cdot \underbrace{\left(\delta \boldsymbol{D}_{0}+c^{b} \boldsymbol{\mathcal { T }}_{\boldsymbol{N}} D \tilde{\boldsymbol{\beta}}\left[\delta \boldsymbol{D}_{0}\right]\right)}_{D \tilde{\boldsymbol{D}}_{0}^{a}\left[\delta \boldsymbol{D}_{0}\right]}+c^{b} \partial_{\boldsymbol{D}_{0}^{b}} e^{b} \cdot \underbrace{\left(\delta \boldsymbol{D}_{0}-c^{a} \boldsymbol{\mathcal { T }}_{\boldsymbol{N}} D \tilde{\boldsymbol{\beta}}\left[\delta \boldsymbol{D}_{0}\right]\right)}_{D \tilde{\boldsymbol{D}}_{0}^{b}\left[\delta \boldsymbol{D}_{0}\right]} .
\end{aligned}
$$

Careful re-arrangement of (66) permits to arrive at the following expression

$$
D e\left[\delta \boldsymbol{D}_{0}\right]=\left(c^{a} \boldsymbol{E}_{0}^{a}+c^{b} \boldsymbol{E}_{0}^{b}\right) \cdot \delta \boldsymbol{D}_{0}+\underbrace{c^{a} c^{b} D \tilde{\boldsymbol{\alpha}}\left[\delta \boldsymbol{D}_{0}\right] \cdot(\llbracket \boldsymbol{P} \rrbracket \boldsymbol{N})}_{=0}+\underbrace{c^{a} c^{b} D \tilde{\boldsymbol{\beta}}\left[\delta \boldsymbol{D}_{0}\right] \cdot\left(\boldsymbol{\mathcal { T }}_{\boldsymbol{N}}^{T} \llbracket \boldsymbol{E}_{0} \rrbracket\right)}_{=0},
$$

where use of the stationary conditions (47) enables to cancel the last two contributions in (67). Finally, equations (65) and (67) enable to obtain the expressions for the effective first PiolaKirchhoff stress tensor $\boldsymbol{P}$ and the effective electric field $\boldsymbol{E}_{0}$ as

$$
\boldsymbol{P}=c^{a} \boldsymbol{P}^{a}+c^{b} \boldsymbol{P}^{b} ; \quad \boldsymbol{E}_{0}=c^{a} \boldsymbol{E}_{0}^{a}+c^{b} \boldsymbol{E}_{0}^{b},
$$

which correspond to the weighted average of their corresponding microscale contributions. 
4.4. Sensitivity of the microscale amplitude vectors with respect to macroscale fields

The stationary conditions (47) can be recalled as

$$
\begin{aligned}
\mathcal{R}_{\boldsymbol{F}}\left(\boldsymbol{F}, \boldsymbol{D}_{0}\right) & =(\delta \boldsymbol{\alpha} \otimes \boldsymbol{N}):\left(\boldsymbol{P}^{a}-\boldsymbol{P}^{b}\right)=0 ; & & \forall \delta \boldsymbol{\alpha} ; \\
\mathcal{R}_{\boldsymbol{D}_{0}}\left(\boldsymbol{F}, \boldsymbol{D}_{0}\right) & =\left(\mathcal{T}_{\boldsymbol{N}} \delta \boldsymbol{\beta}\right) \cdot\left(\boldsymbol{E}_{0}^{a}-\boldsymbol{E}_{0}^{b}\right)=0 ; & & \forall \delta \boldsymbol{\beta},
\end{aligned}
$$

which provide a set of equations where the microscale amplitude vectors can be expressed in an implicit manner in terms of the macroscale deformation gradient and electric displacement. Computation of the sensitivities of $\{\boldsymbol{\alpha}, \boldsymbol{\beta}\}$ with respect to $\left\{\boldsymbol{F}, \boldsymbol{D}_{0}\right\}$ will be accomplished taking advantage of the implicit function theorem. As a result, we can now compute the directional derivative $D \mathcal{R}_{\boldsymbol{F}}[\delta \boldsymbol{F}]$ as

$$
\begin{aligned}
& D \mathcal{R}_{\boldsymbol{F}}[\delta \boldsymbol{F}]=(\delta \boldsymbol{\alpha} \otimes \boldsymbol{N}): \mathcal{C}_{e}^{a}: \underbrace{\left(\delta \boldsymbol{F}+c^{b} D \tilde{\boldsymbol{\alpha}}[\delta \boldsymbol{F}] \otimes \boldsymbol{N}\right)}_{D \tilde{\boldsymbol{F}}^{a}[\delta \boldsymbol{F}]}-(\delta \boldsymbol{\alpha} \otimes \boldsymbol{N}): \mathcal{C}_{e}^{b}: \underbrace{\left(\delta \boldsymbol{F}-c^{a} D \tilde{\boldsymbol{\alpha}}[\delta \boldsymbol{F}] \otimes \boldsymbol{N}\right)}_{D \tilde{\boldsymbol{F}}^{b}[\delta \boldsymbol{F}]} \\
& +(\delta \boldsymbol{\alpha} \otimes \boldsymbol{N}): \mathcal{Q}^{a, T} \cdot \underbrace{\left(c^{b} \boldsymbol{\mathcal { T }}_{\boldsymbol{N}} D \tilde{\boldsymbol{\beta}}[\delta \boldsymbol{F}]\right)}_{D \tilde{\boldsymbol{D}}_{0}^{a}[\delta \boldsymbol{F}]}-(\delta \boldsymbol{\alpha} \otimes \boldsymbol{N}): \mathcal{Q}^{b, T} \cdot \underbrace{\left(-c^{a} \boldsymbol{\mathcal { T }}_{\boldsymbol{N}} D \tilde{\boldsymbol{\beta}}[\delta \boldsymbol{F}]\right)}_{D \tilde{\boldsymbol{D}}_{0}^{b}[\delta \boldsymbol{F}]}=0,
\end{aligned}
$$

where the last equality to zero holds due to the implicit function theorem. A similar derivation can be carried out for the rest of the directional derivatives, namely $D \mathcal{R}_{\boldsymbol{F}}\left[\delta \boldsymbol{D}_{0}\right], D \mathcal{R}_{\boldsymbol{D}_{0}}[\delta \boldsymbol{F}]$ and $D \mathcal{R}_{\boldsymbol{D}_{0}}\left[\delta \boldsymbol{D}_{0}\right]$. After suitable re-arrangement, the directional derivatives can be written as

$$
\begin{aligned}
D \mathcal{R}_{\boldsymbol{F}}[\delta \boldsymbol{F}] & =(\delta \boldsymbol{\alpha} \otimes \boldsymbol{N}): \llbracket \mathcal{C}_{e} \rrbracket: \delta \boldsymbol{F} \\
& +(\delta \boldsymbol{\alpha} \otimes \boldsymbol{N}):\left(c^{b} \mathcal{C}_{e}^{a}+c^{a} \mathcal{C}_{e}^{b}\right):(D \tilde{\boldsymbol{\alpha}}[\delta \boldsymbol{F}] \otimes \boldsymbol{N}) \\
& +(\delta \boldsymbol{\alpha} \otimes \boldsymbol{N}):\left(c^{b} \mathcal{Q}^{a, T}+c^{a} \mathcal{Q}^{b, T}\right) \cdot\left(\mathcal{T}_{\boldsymbol{N}} D \tilde{\boldsymbol{\beta}}[\delta \boldsymbol{F}]\right)=0 ; \\
D \mathcal{R}_{\boldsymbol{D}_{0}}[\delta \boldsymbol{F}] & =\left(\mathcal{T}_{\boldsymbol{N}} \delta \boldsymbol{\beta}\right) \cdot \llbracket \mathcal{Q} \rrbracket: \delta \boldsymbol{F} \\
& +\left(\mathcal{T}_{\boldsymbol{N}} \delta \boldsymbol{\beta}\right) \cdot\left(c^{b} \mathcal{Q}^{a}+c^{a} \mathcal{Q}^{b}\right):(D \tilde{\boldsymbol{\alpha}}[\delta \boldsymbol{F}] \otimes \boldsymbol{N}) \\
& =\left(\mathcal{T}_{\boldsymbol{N}} \delta \boldsymbol{\beta}\right) \cdot\left(c^{b} \boldsymbol{\theta}^{a}+c^{a} \boldsymbol{\theta}^{b}\right)\left(\mathcal{T}_{\boldsymbol{N}} D \tilde{\boldsymbol{\beta}}[\delta \boldsymbol{F}]\right)=0,
\end{aligned}
$$

and

$$
\begin{aligned}
D \mathcal{R}_{\boldsymbol{F}}\left[\delta \boldsymbol{D}_{0}\right] & =(\delta \boldsymbol{\alpha} \otimes \boldsymbol{N}): \llbracket \mathcal{Q}^{T} \cdot \delta \boldsymbol{D}_{0} \\
& +(\delta \boldsymbol{\alpha} \otimes \boldsymbol{N}):\left(c^{b} \mathcal{C}_{e}^{a}+c^{a} \mathcal{C}_{e}^{b}\right):\left(D \tilde{\boldsymbol{\alpha}}\left[\delta \boldsymbol{D}_{0}\right] \otimes \boldsymbol{N}\right) \\
& +(\delta \boldsymbol{\alpha} \otimes \boldsymbol{N}):\left(c^{b} \mathcal{Q}^{a, T}+c^{a} \mathcal{Q}^{b, T}\right) \cdot\left(\mathcal{T}_{\boldsymbol{N}} D \tilde{\boldsymbol{\beta}}\left[\delta \boldsymbol{D}_{0}\right]\right)=0 ; \\
D \mathcal{R}_{\boldsymbol{D}_{0}}\left[\delta \boldsymbol{D}_{0}\right] & =\left(\mathcal{T}_{\boldsymbol{N}} \delta \boldsymbol{\beta}\right) \cdot \llbracket \boldsymbol{\theta} \rrbracket \delta \boldsymbol{D}_{0} \\
& +\left(\mathcal{T}_{\boldsymbol{N}} \delta \boldsymbol{\beta}\right) \cdot\left(c^{b} \mathcal{Q}^{a}+c^{a} \mathcal{Q}^{b}\right):\left(D \tilde{\boldsymbol{\alpha}}\left[\delta \boldsymbol{D}_{0}\right] \otimes \boldsymbol{N}\right) \\
& =\left(\mathcal{T}_{\boldsymbol{N}} \delta \boldsymbol{\beta}\right) \cdot\left(c^{b} \boldsymbol{\theta}^{a}+c^{a} \boldsymbol{\theta}^{b}\right)\left(\mathcal{T}_{\boldsymbol{N}} D \tilde{\boldsymbol{\beta}}\left[\delta \boldsymbol{D}_{0}\right]\right)=0 .
\end{aligned}
$$

It is now possible to combine above equations as follows

$$
\begin{gathered}
-\left[\begin{array}{c}
\llbracket \mathcal{C}_{e, N} \rrbracket \\
\mathcal{T}_{\boldsymbol{N}}^{T} \llbracket \mathcal{Q} \rrbracket
\end{array}\right][: \delta \boldsymbol{F}]=\left[\hat{\mathbb{H}}_{e}^{\boldsymbol{N}}\right]\left[\begin{array}{c}
D \tilde{\boldsymbol{\alpha}}[\delta \boldsymbol{F}] \\
D \tilde{\boldsymbol{\beta}}[\delta \boldsymbol{F}]
\end{array}\right] ; \\
-\left[\begin{array}{c}
\boldsymbol{N} \cdot \llbracket \boldsymbol{\mathcal { Q }} \rrbracket^{T} \\
\mathcal{\mathcal { T }}_{\boldsymbol{N}}^{T} \llbracket \boldsymbol{\theta} \rrbracket
\end{array}\right]\left[\delta \boldsymbol{D}_{0}\right]=\left[\hat{\mathbb{H}}_{e}^{\boldsymbol{N}}\right]\left[\begin{array}{c}
D \tilde{\boldsymbol{\alpha}}\left[\delta \boldsymbol{D}_{0}\right] \\
D \tilde{\boldsymbol{\beta}}\left[\delta \boldsymbol{D}_{0}\right]
\end{array}\right],
\end{gathered}
$$


where $\left(\mathcal{C}_{e, N \bullet}\right)_{i j J}=\left(\mathcal{C}_{e}\right)_{i I j J} N_{I}$. As a result, it is possible to obtain the desired sensitivities as follows

$$
\begin{gathered}
{\left[\begin{array}{c}
D \tilde{\boldsymbol{\alpha}}[\delta \boldsymbol{F}] \\
D \tilde{\boldsymbol{\beta}}[\delta \boldsymbol{F}]
\end{array}\right]=-\left[\hat{\mathbb{H}}_{e}^{\boldsymbol{N}}\right]^{-1}\left[\begin{array}{c}
\llbracket \mathcal{C}_{e, \boldsymbol{N} \bullet} \rrbracket \\
\mathcal{\mathcal { T }}_{\boldsymbol{N}}^{T} \llbracket \mathcal{Q} \rrbracket
\end{array}\right][: \delta \boldsymbol{F}] ;} \\
{\left[\begin{array}{c}
D \tilde{\boldsymbol{\alpha}}\left[\delta \boldsymbol{D}_{0}\right] \\
D \tilde{\boldsymbol{\beta}}\left[\delta \boldsymbol{D}_{0}\right]
\end{array}\right]=-\left[\hat{\mathbb{H}}_{e}^{\boldsymbol{N}}\right]^{-1}\left[\begin{array}{c}
\boldsymbol{N} \cdot \llbracket \mathcal{Q} \rrbracket^{T} \\
\mathcal{T}_{\boldsymbol{N}}^{T} \llbracket \boldsymbol{\theta} \rrbracket
\end{array}\right]\left[\delta \boldsymbol{D}_{0}\right] .}
\end{gathered}
$$

The computation of the sensitivities in (74) is always well-defined due to the invertibility of the Hessian operator $\left[\hat{\mathbb{H}}_{e}^{N}\right]$. Notice that these sensitivities not only are interesting in order to analyse the effect that macroscale fields have on microscale fields, but also are instrumental in order to compute the effective constitutive tensors, as it will be shown in the following section.

\subsection{Effective constitutive tensors}

In order to obtain the effective constitutive tensors, we must first compute the components of the second directional derivative of the internal energy $e\left(\boldsymbol{F}, \boldsymbol{D}_{0}\right)$, namely,

$$
D^{2} e\left[\delta \boldsymbol{F}, \delta \boldsymbol{D}_{0} ; \delta \boldsymbol{F}, \delta \boldsymbol{D}_{0}\right]=D^{2} e[\delta \boldsymbol{F} ; \delta \boldsymbol{F}]+D^{2} e\left[\delta \boldsymbol{F} ; \delta \boldsymbol{D}_{0}\right]+D^{2} e\left[\delta \boldsymbol{D}_{0} ; \delta \boldsymbol{F}\right]+D^{2} e\left[\delta \boldsymbol{D}_{0} ; \delta \boldsymbol{D}_{0}\right]
$$

As an example, we can compute

$$
\begin{aligned}
D^{2} e[\delta \boldsymbol{F} ; \delta \boldsymbol{F}] & =\delta \boldsymbol{F}: c^{a} \mathcal{C}_{e}^{a}: \underbrace{\left(\delta \boldsymbol{F}+c^{b} D \tilde{\boldsymbol{\alpha}}[\delta \boldsymbol{F}] \otimes \boldsymbol{N}\right)}_{D \tilde{\boldsymbol{F}}^{a}[\delta \boldsymbol{F}]}+\delta \boldsymbol{F}: c^{b} \mathcal{C}_{e}^{b}: \underbrace{\left(\delta \boldsymbol{F}-c^{a} D \tilde{\boldsymbol{\alpha}}[\delta \boldsymbol{F}] \otimes \boldsymbol{N}\right)}_{D \tilde{\boldsymbol{F}}^{b}[\delta \boldsymbol{F}]} \\
& +\delta \boldsymbol{F}: c^{a} \boldsymbol{\mathcal { Q }}^{a, T} \cdot \underbrace{\left(c^{b} \mathcal{T}_{\boldsymbol{N}} D \tilde{\boldsymbol{\beta}}[\delta \boldsymbol{F}]\right)}_{D \tilde{\boldsymbol{D}}_{0}^{a}[\delta \boldsymbol{F}]}+\delta \boldsymbol{F}: c^{b} \mathcal{Q}^{b, T} \cdot \underbrace{\left(-c^{a} \boldsymbol{\mathcal { T }}_{\boldsymbol{N}} D \tilde{\boldsymbol{\beta}}[\delta \boldsymbol{F}]\right)}_{D \tilde{\boldsymbol{D}}_{0}^{b}[\delta \boldsymbol{F}]} .
\end{aligned}
$$

A similar derivation can be carried out for the rest of the second directional derivatives featuring in (75) and, after suitable re-arrangement, they can all be written as

$$
\begin{aligned}
D^{2} e[\delta \boldsymbol{F} ; \delta \boldsymbol{F}] & =\delta \boldsymbol{F}:\left(c^{a} \mathcal{C}_{e}^{a}+c^{b} \mathcal{C}_{e}^{b}\right): \delta \boldsymbol{F} \\
& +\delta \boldsymbol{F}: c^{a} c^{b} \llbracket \mathcal{C}_{e} \rrbracket:(D \tilde{\boldsymbol{\alpha}}[\delta \boldsymbol{F}] \otimes \boldsymbol{N})+\delta \boldsymbol{F}: c^{a} c^{b} \llbracket \mathcal{Q} \rrbracket^{T}\left(\mathcal{T}_{\boldsymbol{N}} D \tilde{\boldsymbol{\beta}}[\delta \boldsymbol{F}]\right) ; \\
D^{2} e\left[\delta \boldsymbol{F} ; \delta \boldsymbol{D}_{0}\right] & =\delta \boldsymbol{F}:\left(c^{a} \boldsymbol{\mathcal { Q }}_{e}^{a, T}+c^{b} \boldsymbol{\mathcal { Q }}_{e}^{b, T}\right) \delta \boldsymbol{D}_{0} \\
& +\delta \boldsymbol{F}: c^{a} c^{b} \llbracket \mathcal{C}_{e} \rrbracket:\left(D \tilde{\boldsymbol{\alpha}}\left[\delta \boldsymbol{D}_{0}\right] \otimes \boldsymbol{N}\right)+\delta \boldsymbol{F}: c^{a} c^{b} \llbracket \mathcal{Q}^{T}\left(\mathcal{T}_{\boldsymbol{N}} D \tilde{\boldsymbol{\beta}}\left[\delta \boldsymbol{D}_{0}\right]\right) ; \\
D^{2} e\left[\delta \boldsymbol{D}_{0} ; \delta \boldsymbol{F}\right] & =\delta \boldsymbol{D}_{0} \cdot\left(c^{a} \mathcal{Q}^{a}+c^{b} \boldsymbol{\mathcal { Q }}^{b}\right): \delta \boldsymbol{F} \\
& +\delta \boldsymbol{D}_{0} \cdot c^{a} c^{b} \llbracket \mathcal{Q} \rrbracket:(D \tilde{\boldsymbol{\alpha}}[\delta \boldsymbol{F}] \otimes \boldsymbol{N})+\delta \boldsymbol{D}_{0} \cdot c^{a} c^{b} \llbracket \boldsymbol{\theta} \rrbracket\left(\mathcal{T}_{\boldsymbol{N}} D \tilde{\boldsymbol{\beta}}[\delta \boldsymbol{F}]\right) ; \\
D^{2} e\left[\delta \boldsymbol{D}_{0} ; \delta \boldsymbol{D}_{0}\right] & =\delta \boldsymbol{D}_{0} \cdot\left(c^{a} \boldsymbol{\theta}^{a}+c^{b} \boldsymbol{\theta}^{b}\right) \delta \boldsymbol{D}_{0} \\
& +\delta \boldsymbol{D}_{0} \cdot c^{a} c^{b} \llbracket \mathcal{Q} \rrbracket:\left(D \tilde{\boldsymbol{\alpha}}\left[\delta \boldsymbol{D}_{0}\right] \otimes \boldsymbol{N}\right)+\delta \boldsymbol{D}_{0} \cdot c^{a} c^{b} \llbracket \boldsymbol{\theta} \rrbracket\left(\mathcal{T}_{\boldsymbol{N}} D \tilde{\boldsymbol{\beta}}\left[\delta \boldsymbol{D}_{0}\right]\right) .
\end{aligned}
$$

In order to provide a more insightful representation of (75), above expressions (77) can be combined more effectively into two terms as follows

$$
D^{2} e\left[\delta \boldsymbol{F}, \delta \boldsymbol{D}_{0} ; \delta \boldsymbol{F}, \delta \boldsymbol{D}_{0}\right]=\overline{\mathcal{G}}\left(\delta \boldsymbol{F}, \delta \boldsymbol{D}_{0}\right)+\tilde{\mathcal{H}}\left(\delta \boldsymbol{F}, \delta \boldsymbol{D}_{0}\right)
$$


where $\overline{\mathcal{G}}\left(\delta \boldsymbol{F}, \delta \boldsymbol{D}_{0}\right)$ denotes an average-type term, defined as

$$
\overline{\mathcal{G}}\left(\delta \boldsymbol{F}, \delta \boldsymbol{D}_{0}\right)=\left[\delta \boldsymbol{F}: \delta \boldsymbol{D}_{0} \cdot\right]\left[\overline{\mathbb{H}}_{e}\right]\left[\begin{array}{c}
: \delta \boldsymbol{F} \\
\delta \boldsymbol{D}_{0}
\end{array}\right] ; \quad\left[\overline{\mathbb{H}}_{e}\right]=c^{a}\left[\mathbb{H}_{e}^{a}\right]+c^{b}\left[\mathbb{H}_{e}^{b}\right]=\left[\begin{array}{cc}
\overline{\mathcal{C}}_{e} & \overline{\mathcal{Q}}^{T} \\
\overline{\mathcal{Q}} & \overline{\boldsymbol{\theta}}
\end{array}\right]
$$

and $\overline{\mathcal{H}}\left(\delta \boldsymbol{F}, \delta \boldsymbol{D}_{0}\right)$ denotes a jump-type contribution, defined as

$$
\tilde{\mathcal{H}}\left(\delta \boldsymbol{F}, \delta \boldsymbol{D}_{0}\right)=\left[\delta \boldsymbol{F}: \delta \boldsymbol{D}_{0} \cdot\right] c^{a} c^{b}\left[\begin{array}{cc}
\llbracket \mathcal{C}_{e, \bullet N} \rrbracket & \llbracket \mathcal{Q} \rrbracket^{T} \mathcal{T}_{\boldsymbol{N}} \\
\llbracket \mathcal{Q} \rrbracket \boldsymbol{N} & \llbracket \boldsymbol{\theta} \rrbracket \boldsymbol{\mathcal { T }}_{\boldsymbol{N}}
\end{array}\right]\left[\begin{array}{c}
D \tilde{\boldsymbol{\alpha}}[\delta \boldsymbol{F}]+D \tilde{\boldsymbol{\alpha}}\left[\delta \boldsymbol{D}_{0}\right] \\
D \tilde{\boldsymbol{\beta}}[\delta \boldsymbol{F}]+D \tilde{\boldsymbol{\beta}}\left[\delta \boldsymbol{D}_{0}\right]
\end{array}\right],
$$

where $\left(\mathcal{C}_{e, \bullet N}\right)_{i I j}=\left(\mathcal{C}_{e}\right)_{i I j J} N_{J}$. Moreover, it is now possible to further elaborate on the last term of the right-hand side of above equation (80) by means of the sensitivities computed in (74). Specifically,

$$
\left[\begin{array}{c}
D \tilde{\boldsymbol{\alpha}}[\delta \boldsymbol{F}]+D \tilde{\boldsymbol{\alpha}}\left[\delta \boldsymbol{D}_{0}\right] \\
D \tilde{\boldsymbol{\beta}}[\delta \boldsymbol{F}]+D \tilde{\boldsymbol{\beta}}\left[\delta \boldsymbol{D}_{0}\right]
\end{array}\right]=-\left[\hat{\mathbb{H}}_{e}^{\boldsymbol{N}}\right]^{-1}\left[\begin{array}{cc}
\llbracket \mathcal{C}_{e, \boldsymbol{N}} \rrbracket & \boldsymbol{N} \cdot \llbracket \mathcal{Q} \rrbracket^{T} \\
\mathcal{T}_{\boldsymbol{N}}^{T} \llbracket \mathcal{Q} \rrbracket & \mathcal{T}_{\boldsymbol{N}}^{T} \llbracket \boldsymbol{\theta} \rrbracket
\end{array}\right]\left[\begin{array}{l}
: \delta \boldsymbol{F} \\
\delta \boldsymbol{D}_{0}
\end{array}\right]
$$

Thus, substitution of equation (81) into (80) yields

$$
\begin{aligned}
& \tilde{\mathcal{H}}\left(\delta \boldsymbol{F}, \delta \boldsymbol{D}_{0}\right)=-\left[\delta \boldsymbol{F}: \delta \boldsymbol{D}_{0} \cdot\right]\left[\begin{array}{cc}
\llbracket \mathcal{C}_{e, \bullet N} \rrbracket & \llbracket \mathcal{Q} \rrbracket^{T} \mathcal{T}_{\boldsymbol{N}} \\
\llbracket \mathcal{Q} \rrbracket \boldsymbol{N} & \llbracket \boldsymbol{\theta} \rrbracket \mathcal{T}_{\boldsymbol{N}}
\end{array}\right] c^{a} c^{b}\left[\hat{\mathbb{H}}_{e}^{N}\right]^{-1}\left[\begin{array}{cc}
\llbracket \mathcal{C}_{e, \boldsymbol{N}} \rrbracket & \boldsymbol{N} \cdot \llbracket \mathcal{Q}^{T} \rrbracket^{T} \\
\mathcal{T}_{\boldsymbol{N}}^{T} \llbracket \mathcal{Q} \rrbracket & \mathcal{T}_{\boldsymbol{N}}^{T} \llbracket \boldsymbol{\theta} \rrbracket
\end{array}\right]\left[\begin{array}{l}
: \delta \boldsymbol{F} \\
\delta \boldsymbol{D}_{0}
\end{array}\right] \\
& =-\left[\delta \boldsymbol{F}: \delta \boldsymbol{D}_{0} \cdot\right]\left[\tilde{\mathbb{H}}_{e}\right]\left[\begin{array}{l}
: \delta \boldsymbol{F} \\
\delta \boldsymbol{D}_{0}
\end{array}\right]
\end{aligned}
$$

where

$$
\left[\tilde{\mathbb{H}}_{e}\right]=\left[\begin{array}{cc}
\tilde{\mathcal{C}_{e}} & \tilde{\mathcal{Q}}^{T} \\
\tilde{\mathcal{Q}} & \tilde{\boldsymbol{\theta}}
\end{array}\right] ; \quad\left[\tilde{\mathbb{H}}_{e}\right]=\left[\begin{array}{cc}
\llbracket \mathcal{C}_{e, \boldsymbol{N}} \rrbracket & \llbracket \mathcal{Q} \rrbracket^{T} \mathcal{\mathcal { T }}_{\boldsymbol{N}} \\
\llbracket \mathcal{Q} \rrbracket \boldsymbol{N} & \llbracket \boldsymbol{\theta} \rrbracket \mathcal{T}_{\boldsymbol{N}}
\end{array}\right]\left[\check{\mathbb{H}}_{e}^{\boldsymbol{N}}\right]^{-1}\left[\begin{array}{cc}
\llbracket \mathcal{C}_{e, \boldsymbol{N}} \rrbracket & \boldsymbol{N} \cdot \llbracket \mathcal{Q} \rrbracket^{T} \\
\mathcal{T}_{\boldsymbol{N}}^{T} \llbracket \mathcal{Q} \rrbracket & \mathcal{T}_{\boldsymbol{N}}^{T} \llbracket \boldsymbol{\theta} \rrbracket
\end{array}\right]
$$

where $\left[\check{\mathbb{H}}_{e}^{N}\right]=\left[\hat{\mathbb{H}}_{e}^{N}\right] /\left(c^{a} c^{b}\right)$ represents a harmonic mean type of Hessian with an expression as

$$
\left[\check{\mathbb{H}}_{e}^{\boldsymbol{N}}\right]=\left[\begin{array}{cc}
\check{\mathcal{C}}_{e, \mathbf{N} N} & \boldsymbol{N} \cdot \check{\mathcal{Q}}^{T} \mathcal{T}_{\boldsymbol{N}} \\
\mathcal{T}_{\boldsymbol{N}}^{T}{ }^{T} \mathcal{\mathcal { Q }} \boldsymbol{N} & \mathcal{T}_{\boldsymbol{N}}^{T} \check{\boldsymbol{\theta}}^{\mathcal{T}_{\boldsymbol{N}}}
\end{array}\right] ; \quad\left[\check{\mathbb{H}}_{e}\right]=\frac{1}{c^{a}}\left[\mathbb{H}_{e}^{a}\right]+\frac{1}{c^{b}}\left[\mathbb{H}_{e}^{b}\right]=\left[\begin{array}{cc}
\check{\mathcal{C}}_{e} & \check{\mathcal{Q}}^{T} \\
\check{\mathcal{Q}} & \check{\boldsymbol{\theta}}
\end{array}\right]
$$

Finally, we arrive at

$$
D^{2} e\left[\delta \boldsymbol{F}, \delta \boldsymbol{D}_{0} ; \delta \boldsymbol{F}, \delta \boldsymbol{D}_{0}\right]=\left[\delta \boldsymbol{F}: \delta \boldsymbol{D}_{0} \cdot\right]\left(\left[\overline{\mathbb{H}}_{e}\right]-\left[\tilde{\mathbb{H}}_{e}\right]\right)\left[\begin{array}{l}
: \delta \boldsymbol{F} \\
\delta \boldsymbol{D}_{0}
\end{array}\right]
$$

with $\left[\overline{\mathbb{H}}_{e}\right]$ and $\left[\tilde{\mathbb{H}}_{e}\right]$ defined in (79) and (83)-(84), respectively. Hence, equation (85) reveals that the effective constitutive tensors $\left\{\mathcal{C}_{e}, \mathcal{Q}, \boldsymbol{\theta}\right\}$ can be additively decomposed into two distinct contributions: a simple average of the individual phases in the microscale, and an additional term associated with the sensitivities of the (micro) amplitude vectors $\{\boldsymbol{\alpha}, \boldsymbol{\beta}\}$ with respect to the macroscopic deformation gradient tensor $\boldsymbol{F}$ and electric displacement field $\boldsymbol{D}_{0}$. We will demonstrate that the first contribution (simple averaging of micro-constituents) complies with the ellipticity condition (10) provided that the internal energy functionals $e^{a}\left(\boldsymbol{F}^{a}, \boldsymbol{D}_{0}^{a}\right)$ and $e^{b}\left(\boldsymbol{F}^{b}, \boldsymbol{D}_{0}^{b}\right)$ are CMV compliant. However, the second term can potentially induce loss of ellipticity of the homogenised tangent operator. Specifically,

$$
\begin{aligned}
& \mathcal{C}_{e}=\underbrace{\overline{\mathcal{C}}_{e}\left(c^{a}, c^{b}, \boldsymbol{F}^{a}, \boldsymbol{F}^{b}, \boldsymbol{D}_{0}^{a}, \boldsymbol{D}_{0}^{b}\right)}_{\text {(Average) ellipticity-preserving contr. }}-\underbrace{\tilde{\mathcal{C}}_{e}\left(c^{a}, c^{b}, \boldsymbol{F}^{a}, \boldsymbol{F}^{b}, \boldsymbol{D}_{0}^{a}, \boldsymbol{D}_{0}^{b}\right)}_{\text {Macro ellipticity-breaking contr. }} ; \\
& \mathcal{Q}=\underbrace{\overline{\mathcal{Q}}\left(c^{a}, c^{b}, \boldsymbol{F}^{a}, \boldsymbol{F}^{b}, \boldsymbol{D}_{0}^{a}, \boldsymbol{D}_{0}^{b}\right)}_{\text {(Average) ellipticity-preserving contr. }}-\underbrace{\tilde{\mathcal{Q}}\left(c^{a}, c^{b}, \boldsymbol{F}^{a}, \boldsymbol{F}^{b}, \boldsymbol{D}_{0}^{a}, \boldsymbol{D}_{0}^{b}\right)}_{\text {Macro ellipticity-breaking contr. }} ; \\
& \boldsymbol{\theta}=\underbrace{\overline{\boldsymbol{\theta}}\left(c^{a}, c^{b}, \boldsymbol{F}^{a}, \boldsymbol{F}^{b}, \boldsymbol{D}_{0}^{a}, \boldsymbol{D}_{0}^{b}\right)}_{\text {(Average) ellipticity-preserving contr. }}-\underbrace{\tilde{\boldsymbol{\theta}}\left(c^{a}, c^{b}, \boldsymbol{F}^{a}, \boldsymbol{F}^{b}, \boldsymbol{D}_{0}^{a}, \boldsymbol{D}_{0}^{b}\right)}_{\text {Macro ellipticity-breaking contr. }} .
\end{aligned}
$$


As for the average-type term $\overline{\mathcal{G}}\left(\delta \boldsymbol{F}, \delta \boldsymbol{D}_{0}\right)$, adopting $\delta \boldsymbol{F}=\boldsymbol{u} \otimes \boldsymbol{V}$ and $\delta \boldsymbol{D}_{0}=\boldsymbol{V}_{\perp}$, it is immediate to realise that

$$
\overline{\mathcal{G}}\left(\boldsymbol{u} \otimes \boldsymbol{V}, \boldsymbol{V}_{\perp}\right)=\left[\boldsymbol{u} \otimes \boldsymbol{V}: \boldsymbol{V}_{\perp} \cdot\right]\left[\overline{\mathbb{H}}_{e}\right]\left[\begin{array}{c}
: \boldsymbol{u} \otimes \boldsymbol{V} \\
\boldsymbol{V}_{\perp}
\end{array}\right]>0 ; \quad \forall \boldsymbol{u}, \boldsymbol{V}, \boldsymbol{V}_{\perp},
$$

provided that the phases $a$ and $b$ are CMV compliant. Proceeding in an analogous manner with the jump-type contribution, it results in

$$
\begin{aligned}
& \tilde{\mathcal{H}}\left(\boldsymbol{u} \otimes \boldsymbol{V}, \boldsymbol{V}_{\perp}\right)=-\left[\boldsymbol{u} \otimes \boldsymbol{V}: \boldsymbol{V}_{\perp} \cdot\right]\left[\tilde{\mathbb{H}}_{e}\right]\left[\begin{array}{c}
: \boldsymbol{u} \otimes \boldsymbol{V} \\
\boldsymbol{V}_{\perp}
\end{array}\right] \\
& =-\left[\boldsymbol{u} \otimes \boldsymbol{V}: \boldsymbol{V}_{\perp} \cdot\right]\left[\begin{array}{cc}
\llbracket \mathcal{C}_{e, \bullet N} \rrbracket & \llbracket \mathcal{Q} \rrbracket^{T} \mathcal{T}_{\boldsymbol{N}} \\
\llbracket \mathcal{Q} \rrbracket \boldsymbol{N} & \llbracket \boldsymbol{\theta} \rrbracket \mathcal{T}_{\boldsymbol{N}}
\end{array}\right]\left[\check{\mathbb{H}}_{e}^{N}\right]^{-1}\left[\begin{array}{cc}
\llbracket \mathcal{C}_{e, \boldsymbol{N}} \rrbracket & \boldsymbol{N} \cdot \llbracket \mathcal{Q} \rrbracket^{T} \\
\mathcal{T}_{\boldsymbol{N}}^{T} \llbracket \mathcal{Q} \rrbracket & \mathcal{T}_{\boldsymbol{N}}^{T} \llbracket \boldsymbol{\theta} \rrbracket
\end{array}\right]\left[\begin{array}{c}
: \boldsymbol{u} \otimes \boldsymbol{V} \\
\boldsymbol{V}
\end{array}\right]
\end{aligned}
$$

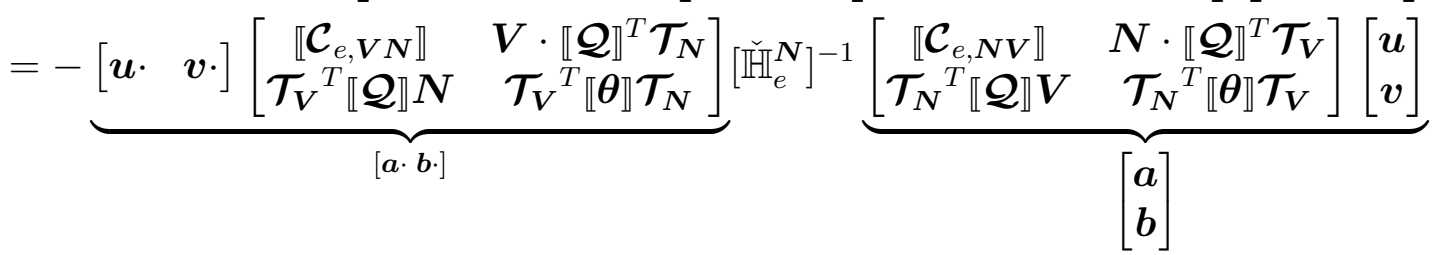

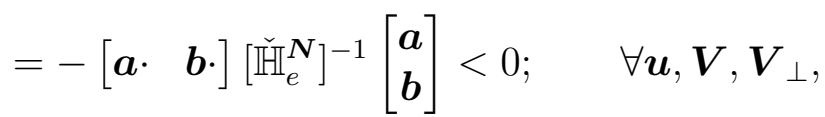

where use of the projection operator $\mathcal{T}_{\boldsymbol{V}}$ (i.e., $\boldsymbol{V}_{\perp}=\mathcal{T}_{\boldsymbol{V}} \boldsymbol{v}$ ) has been made in the third line above, and vectors $\boldsymbol{a}$ and $\boldsymbol{b}$ have been introduced. It is immediate to realise that above term is negative due to the positive definiteness of the Hessian $\left[\check{\mathbb{H}}_{e}^{N}\right]$. It is at this juncture when it is important to emphasise the use that we have made of Remark 4 in order to drastically simplify the algebra associated with above computation. In summary,

(a) the consideration of CMV (and thus rank-one convex) constitutive models for the microscale constituents guarantees that the analytical homogenisation (computation of the micro-amplitude vectors $\{\boldsymbol{\alpha}, \boldsymbol{\beta}\})$ can be accomplished for any value of the macroscopic fields $\left\{\boldsymbol{F}, \boldsymbol{D}_{0}\right\}$;

(b) the high nonlinearity associated with the microscopic problem can result in an intricate dependence of both $\{\boldsymbol{\alpha}, \boldsymbol{\beta}\}$ upon $\left\{\boldsymbol{F}, \boldsymbol{D}_{0}\right\}$, permitting the contributions $\left\{\tilde{\mathcal{C}}_{e}, \tilde{\mathcal{Q}}, \tilde{\boldsymbol{\theta}}\right\}$ in $(86)$ to potentially induce an overall loss of ellipticity (or rank-one convexity condition) of the effective tensors $\left\{\mathcal{C}_{e}, \mathcal{Q}, \boldsymbol{\theta}\right\}$, by neutralising, and even counterbalancing, the unconditionally rank-one convex contribution resulting from the averaging of the micro-constituents $\left\{\overline{\mathcal{C}}_{e}, \overline{\mathcal{Q}}, \overline{\boldsymbol{\theta}}\right\}$.

Crucially, the mathematical connotations associated with point (b) above imply that the homogenisation procedure can eventually jeopardise the well-posedness of the system in (4). From a physical standpoint, as shown in [5], the onset of loss of ellipticity corresponds with the development of localised deformations, and it is an expected phenomenon related to the development of long wavelength diffuse modes in the microscale. Indeed, loss of ellipticity can occur in the solely mechanical case, even when (polyconvex) Neo-Hookean strain energies are considered for the constitutive models of the micro-constituents of layered composites (see Reference [24]). Suitable relaxation techniques, such as rank-one convexification, can be applied [24] in order to restore the mathematical well-posedness of the problem. Notwithstanding, the latter is out of the scope of this paper. In the numerical examples considered in this paper, loss of ellipticity has not been observed. 


\section{Variational principles and Finite Element implementation}

The most widely used variational principle, from which the weak forms associated with the PDEs in equation (4) emerge, is the following

$$
\Pi(\phi, \varphi)=\inf _{\phi} \sup _{\varphi}\left\{\int_{\mathcal{B}_{0}} \Psi\left(\boldsymbol{F},-\nabla_{0} \varphi\right) d V-\Pi_{\text {ext }}^{m}(\phi)-\Pi_{\text {ext }}^{e}(\varphi)\right\},
$$

where $\Pi_{\text {ext }}^{m}$ and $\Pi_{\text {ext }}^{e}$ represent the external energetic contributions, defined as

$$
\Pi_{\mathrm{ext}}^{m}(\boldsymbol{\phi})=\int_{\mathcal{B}_{0}} \boldsymbol{f}_{0} \cdot \boldsymbol{\phi} d V+\int_{\partial_{\boldsymbol{t}} \mathcal{B}_{0}} \boldsymbol{t}_{0} \cdot \boldsymbol{\phi} d A ; \quad \Pi_{\mathrm{ext}}^{e}(\varphi)=-\int_{\mathcal{B}_{0}} \rho_{0}^{e} \varphi d V-\int_{\partial_{\omega} \mathcal{B}_{0}} \omega_{0}^{e} \varphi d A
$$

Notice that in our work we advocate for constitutive models defined in terms of the homogenised or effective internal energy $e\left(\boldsymbol{F}, \boldsymbol{D}_{0}\right)$ (43), and hence an explicit definition of the homogenised Helmholtz functional $\Psi\left(\boldsymbol{F}, \boldsymbol{E}_{0}\right)$ is no longer necessarily available. In order to circumvent this drawback, we make use of the Legendre transformation in (28) and re-express the homogenised functional $\Psi\left(\boldsymbol{F}, \boldsymbol{E}_{0}\right)$ in (89) in terms of the homogenised internal energy functional $e\left(\boldsymbol{F}, \boldsymbol{D}_{0}\left(\boldsymbol{F}, \boldsymbol{E}_{0}\right)\right)$, yielding the following equivalent variational principle

$$
\widetilde{\Pi}(\boldsymbol{\phi}, \varphi)=\left\{\begin{array}{l}
\inf _{\boldsymbol{\phi}} \sup _{\varphi}\left\{\int_{\mathcal{B}_{0}} e\left(\boldsymbol{F}, \boldsymbol{D}_{0}\right) d V+\int_{\mathcal{B}_{0}} \boldsymbol{D}_{0} \cdot \nabla_{0} \varphi d V-\Pi_{\mathrm{ext}}^{m}(\boldsymbol{\phi})-\Pi_{\mathrm{ext}}^{e}(\varphi)\right\} ; \\
\text { s.t. } \quad-\boldsymbol{\nabla}_{0} \varphi=\partial_{\boldsymbol{D}_{0}} e\left(\boldsymbol{F}, \boldsymbol{D}_{0}\right) .
\end{array}\right.
$$

Then we introduce the functional spaces for the fields $\{\phi, \varphi\} \in \mathbb{V}^{\phi} \times \mathbb{V}^{\varphi}$ and the test functions $\{\delta \phi, \delta \varphi\} \in \mathbb{V}_{0}^{\phi} \times \mathbb{V}_{0}^{\varphi}$ featuring in the variational principle (91) as

$$
\begin{array}{rlrl}
\mathbb{V}^{\phi} & =\left\{\phi: \mathcal{B}_{0} \rightarrow \mathbb{R}^{3} ; \quad(\phi)_{i} \in H^{1}\left(\mathcal{B}_{0}\right) \mid J>0\right\} ; & \mathbb{V}^{\varphi} & =\left\{\varphi: \mathcal{B}_{0} \rightarrow \mathbb{R}^{1} ; \quad \varphi \in H^{1}\left(\mathcal{B}_{0}\right)\right\} ; \\
\mathbb{V}_{0}^{\phi} & =\left\{\forall \phi \in \mathbb{V}^{\phi} ; \quad \phi=0 \text { on } \partial_{\phi} \mathcal{B}_{0}\right\} ; & \mathbb{V}_{0}^{\varphi}=\left\{\forall \varphi \in \mathbb{V}^{\varphi} ; \quad \varphi=0 \text { on } \partial_{\varphi} \mathcal{B}_{0}\right\} .
\end{array}
$$

Clearly, the stationary conditions of the variational principle $\widetilde{\Pi}$ in (91) yield the weak forms associated with the PDEs in equation (4), namely

$$
\begin{aligned}
& D \widetilde{\Pi}[\delta \phi]=\int_{\mathcal{B}_{0}} \boldsymbol{P}: D \boldsymbol{F}[\delta \boldsymbol{\phi}] d V-\int_{\mathcal{B}_{0}} \boldsymbol{f}_{0} \cdot \delta \boldsymbol{\phi} d V-\int_{\partial_{t} \mathcal{B}_{0}} \boldsymbol{t}_{0} \cdot \delta \boldsymbol{\phi} d A=0 ; \\
& D \widetilde{\Pi}[\delta \varphi]=\int_{\mathcal{B}_{0}} \boldsymbol{D}_{0} \cdot \nabla_{0} \delta \varphi d V+\int_{\mathcal{B}_{0}} \rho_{0}^{e} \delta \varphi d V+\int_{\partial_{\omega} \mathcal{B}_{0}} \omega_{0}^{e} \delta \varphi d A=0,
\end{aligned}
$$

with $\boldsymbol{D}_{0}$ obtained from constraint in (91) and with $\boldsymbol{P}$ obtained as in (6)a. A Newton-Raphson scheme can be used for the solution of the weak forms in (93), which implies the following linearisation with respect to incremental fields $\Delta \phi \in \mathbb{V}_{0}^{\phi}$ and $\Delta \varphi \in \mathbb{V}_{0}^{\varphi}$

$$
0=D \widetilde{\Pi}[\delta \phi]+D \widetilde{\Pi}[\delta \varphi]+D^{2} \widetilde{\Pi}[\delta \phi ; \Delta \phi]+D^{2} \widetilde{\Pi}[\delta \phi ; \Delta \varphi]+D^{2} \widetilde{\Pi}[\delta \varphi ; \Delta \phi]+D^{2} \widetilde{\Pi}[\delta \varphi ; \Delta \varphi]
$$

with

$$
\begin{array}{rlrl}
D^{2} \widetilde{\Pi}[\delta \phi ; \Delta \phi] & =\int_{\mathcal{B}_{0}} \nabla_{0} \delta \phi: \mathcal{C}: \nabla_{0} \Delta \phi d V ; & D^{2} \widetilde{\Pi}[\delta \phi ; \Delta \varphi] & =\int_{\mathcal{B}_{0}}\left(\boldsymbol{\nabla}_{0} \delta \phi: \mathcal{P}^{T}\right) \cdot \nabla_{0} \Delta \varphi d V ; \\
D^{2} \widetilde{\Pi}[\delta \varphi ; \Delta \phi] & =\int_{\mathcal{B}_{0}} \nabla_{0} \delta \varphi \cdot\left(\mathcal{P}: \nabla_{0} \Delta \phi\right) d V ; & D^{2} \widetilde{\Pi}[\delta \varphi ; \Delta \varphi]=-\int_{\mathcal{B}_{0}} \boldsymbol{\nabla}_{0} \delta \varphi \cdot\left(\boldsymbol{\epsilon} \boldsymbol{\nabla}_{0} \Delta \varphi\right) d V,
\end{array}
$$


with the homogenised tensors $\{\mathcal{C}, \mathcal{P}, \boldsymbol{\epsilon}\}$ related to their homogenised internal energy-based counterparts $\left\{\mathcal{C}_{e}, \mathcal{Q}, \boldsymbol{\theta}\right\}$ in (86) through the relationship in equation (32). Equation (94) permits the update of the solution fields $\phi \in \mathbb{V}^{\phi}$ and $\varphi \in \mathbb{V}^{\varphi}$ at a given Newton-Raphson iteration $k+1$ as

$$
\phi^{k+1}=\phi^{k}+\Delta \phi ; \quad \varphi^{k+1}=\varphi^{k}+\Delta \varphi .
$$

As standard in finite elements, the domain $\mathcal{B}_{0}$ described in Section 2.1, representing the EAP, is sub-divided into a finite set of non-overlapping elements $e \in \mathbb{E}$ as follows

$$
\mathcal{B}_{0} \approx \mathcal{B}_{0}^{h}=\bigcup_{e \in \mathbb{E}} \mathcal{B}_{0}^{e}
$$

The unknown fields $\{\phi, \varphi\}$, and the test functions $\{\delta \phi, \delta \varphi\}$ featuring in $D \widetilde{\Pi}[\delta \phi], D \widetilde{\Pi}[\delta \varphi]$ in (93), are discretised using the functional spaces $\mathbb{V}^{\phi^{h}} \times \mathbb{V}^{\varphi^{h}}$ and $\mathbb{V}_{0}^{\phi^{h}} \times \mathbb{V}_{0}^{\varphi^{h}}$, respectively, defined as

$$
\begin{array}{lll}
\mathbb{V}^{\phi^{h}}=\left\{\phi \in \mathbb{V}^{\phi} ;\left.\quad \phi^{h}\right|_{\mathcal{B}_{0}^{e}}=\sum_{a=1}^{n_{\text {node }}^{\phi}} N_{a}^{\phi} \phi_{a}\right\} ; & \mathbb{V}^{\varphi^{h}}=\left\{\varphi \in \mathbb{V}^{\varphi} ;\left.\quad \varphi^{h}\right|_{\mathcal{B}_{0}^{e}}=\sum_{a=1}^{n_{\text {node }}^{\varphi}} N_{a}^{\varphi} \varphi_{a}\right\} ; \\
\mathbb{V}_{0}^{\phi^{h}}=\left\{\forall \phi \in \mathbb{V}^{\phi^{h}} ; \quad \phi=0 \text { on } \partial_{\phi} \mathcal{B}_{0}\right\} ; & \mathbb{V}_{0}^{\varphi^{h}}=\left\{\forall \varphi \in \mathbb{V}^{\varphi^{h}} ; \quad \varphi=0 \text { on } \partial_{\varphi} \mathcal{B}_{0}\right\},
\end{array}
$$

where, for any field $\mathcal{Y} \in\{\phi, \varphi\}, n_{\text {node }}^{\mathcal{Y}}$ denotes the number of nodes per element of the discretisation associated with the field $\mathcal{Y}$; and $N_{a}^{\mathcal{Y}}: \mathcal{B}_{0}^{e} \rightarrow \mathbb{R}$, the $a^{\text {th }}$ shape function used for the interpolation of $\mathcal{Y}$. In addition, $\mathcal{Y}_{a}$ represents the value of the field $\mathcal{Y}$ at the $a^{\text {th }}$ node of a given finite element. Consideration of the functional spaces in (98) enables the weak forms $D \widetilde{\Pi}[\delta \phi]$, $D \widetilde{\Pi}[\delta \varphi]$ in $(93)$ to be written in terms of their associated elemental residual contributions, namely

$$
D \widetilde{\Pi}[\delta \phi]=\sum_{e=1}^{N} \delta \phi_{a} \cdot \boldsymbol{R}_{a, e}^{\phi} ; \quad D \widetilde{\Pi}[\delta \varphi]=\sum_{e=1}^{N} \delta \varphi_{a} R_{a, e}^{\varphi},
$$

where $N$ denotes the number of elements for the underlying discretisation, and where each of the residual contributions $\boldsymbol{R}_{a, e}^{\phi}$ and $R_{a, e}^{\varphi}$ can be expressed as ${ }^{9}$

$$
\boldsymbol{R}_{a, e}^{\phi}=\int_{\mathcal{B}_{0}^{e}} \boldsymbol{P} \nabla_{0} N_{a}^{\phi} d V+\int_{\mathcal{B}_{0}^{e}} N_{a}^{\phi} \boldsymbol{f}_{0} d V ; \quad R_{a, e}^{\varphi}=\int_{\mathcal{B}_{0}^{e}} \boldsymbol{D}_{0} \cdot \nabla_{0} N_{a}^{\varphi} d V+\int_{\mathcal{B}_{0}^{e}} N_{a}^{\varphi} \rho^{e} d V .
$$

Discretisation of the test functions and incremental fields permits equation (95) to be written in terms of their associated elemental stiffness contributions, namely

$$
\begin{aligned}
D^{2} \widetilde{\Pi}[\delta \phi, \Delta \phi] & =\sum_{e=1}^{N} \delta \phi_{a} \cdot \boldsymbol{K}_{a b, e}^{\phi \phi} \Delta \boldsymbol{\phi}_{b} ; & D^{2} \widetilde{\Pi}[\delta \phi, \Delta \varphi] & =\sum_{e=1}^{N} \delta \boldsymbol{\phi}_{a} \cdot \boldsymbol{K}_{a b, e}^{\phi \varphi} \Delta \varphi_{b} ; \\
D^{2} \widetilde{\Pi}[\delta \varphi, \Delta \phi] & =\sum_{e=1}^{N} \delta \varphi_{a} \cdot \boldsymbol{K}_{a b, e}^{\varphi \phi} \Delta \boldsymbol{\phi}_{b} ; & D^{2} \widetilde{\Pi}[\delta \varphi, \Delta \varphi] & =\sum_{e=1}^{N} \delta \varphi_{a} K_{a b, e}^{\varphi \varphi} \Delta \varphi_{b},
\end{aligned}
$$

where each of the stiffness contributions is expressed as

$$
\begin{aligned}
\left(\boldsymbol{K}_{a b, e}^{\phi \phi}\right)_{i j} & =\int_{\mathcal{B}_{0}^{e}}\left(\nabla_{0} N_{a}^{\phi}\right)_{I}\left(\nabla_{0} N_{b}^{\phi}\right)_{J} \mathcal{C}_{i I j J} d V ; & \left(\boldsymbol{K}_{a b, e}^{\phi \varphi}\right)_{i} & =\int_{\mathcal{B}_{0}^{e}}\left(\nabla_{0} N_{a}^{\phi}\right)_{I}\left(\nabla_{0} N_{b}^{\varphi}\right)_{J}(\mathcal{P})_{i I J}^{T} d V ; \\
\boldsymbol{K}_{a b, e}^{\varphi \phi} & =\left(\boldsymbol{K}_{a b, e}^{\varphi \phi}\right)^{T} ; & K_{a b, e}^{\varphi \varphi} & =-\int_{\mathcal{B}_{0}^{e}} \nabla_{0} N_{a}^{\varphi} \cdot \boldsymbol{\epsilon} \boldsymbol{\nabla}_{0} N_{b}^{\varphi} d V .
\end{aligned}
$$

\footnotetext{
${ }^{9}$ For simplicity, the external contributions on the boundary of the continuum associated with $\boldsymbol{t}_{0}$ and $\omega_{0}^{e}$ have not been included in (100).
} 


\section{Numerical examples}

In this Section, three numerical examples will be presented in order to assess the capability and robustness of the proposed computational framework. The first numerical example, restricted to the case of homogeneous deformation, circumvents the need to use a Finite Element discretisation and studies the behaviour, at a local level, of the homogenised constitutive model. A comprehensive study will be conducted, where the influence that laminate orientation, contrast of material properties, microscopic material properties and operational range (i.e., deformation and electric field) has on the response of the composite will be presented. The second example abandons the assumption of uniform deformation and explores the use of Finite Elements in the context of a rectangular flexible DE composite film displaying complex actuation patterns through application of electrical stimuli. In addition, a thorough study will be conducted, where different microscopic arrangements will be shown to lead to potentially very different actuating configurations (namely bending, torsion, stretching). Finally, the third numerical example explores recent findings reported in [32] about the onset of buckling-type instabilities in DE composites, and this will be studied for a square membrane configuration. The various numerical examples will help to emphasise the importance of in silico simulations to better engineer the design of complex multi-layered laminated composites. Moreover, the optimum composite combination will be shown to be strongly dependent upon the operational range, both in terms of deformation and electric field.

\subsection{Numerical example 1: homogeneous electro-deformation of a multi-layered DE composite}

Through this example we aim to:

- Capture the differences in performance, both at pre- and post-instability regions, for a rank-one DE laminated composite when considering two distinctly different microstructure arrangements.

- Appreciate the importance of using arc-length techniques in order to bypass instability regions and harness actuator performance beyond the moderate regime.

- Monitor the effect that microstructure has in the propagation of shear wave speeds for two rank-one laminated compositions, especially when considering high contrast nearly incompressible constituents.

- Study the evolution of the acoustic tensor as a function of deformation and electric field in order to assess the possible loss of macroscopic stability.
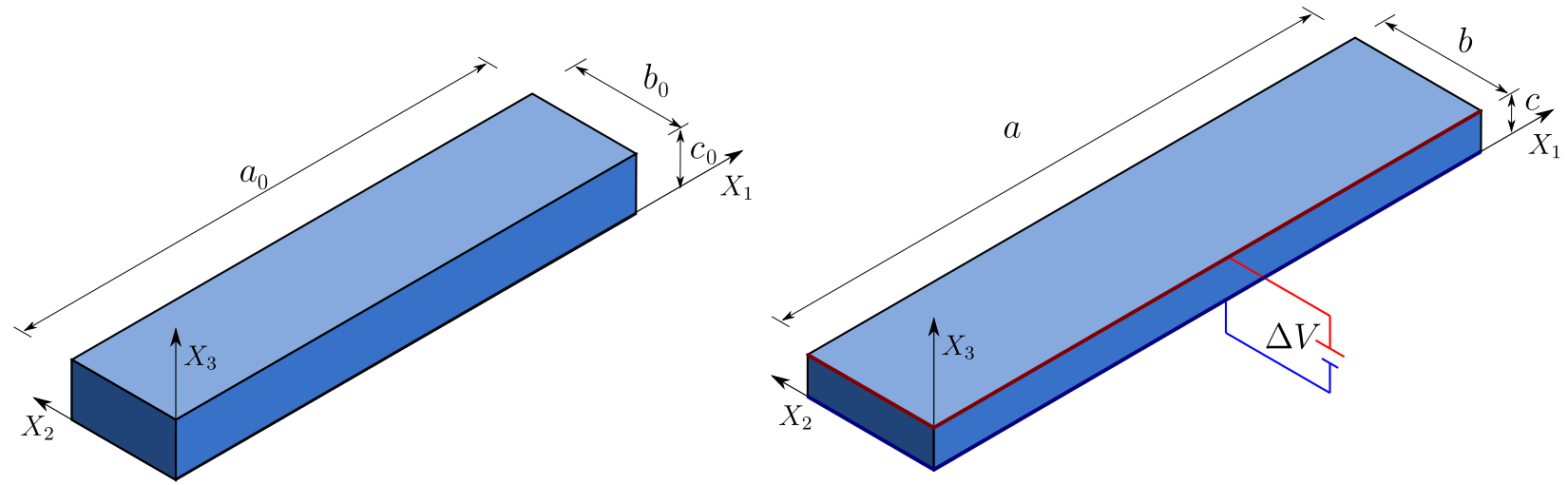

Figure 4: Numerical example 1. Experimental set-up. The application of a uniform electric field along the $O X_{3}$ direction causes a stretch of the DE laminated composite along $O X_{1}$ direction. 
We consider a prototypical set-up very similar to that already explored by several authors in the past, both numerically [27, 77] and experimentally [79]. This consists of a rank-one DE laminated film, such as the one depicted in Figure 4, which is subjected to a homogeneous state of deformation and electric field. Two electrodes are placed at opposite faces of the film and an externally controlled Lagrangian electric field $\boldsymbol{E}_{\mathbf{0}}$ is applied across and orientated along the $O X_{3}$ axis, whilst maintaining stress free conditions, in turn generating a state of uniform deformation and electric displacement across the film. This uniform state of deformation and electric field is exploited in order to study the response of the DE rank-one laminate from a local point of view, without the need to resort to a finite element discretisation. As a result, in the absence of any further loads and electric charges, the homogeneous solution to this problem corresponds to the stationary points $\left\{\boldsymbol{F}^{*}, \boldsymbol{D}_{0}^{*}\right\}$ of the Helmholtz's free energy functional defined as

$$
\Pi\left(\boldsymbol{F}^{*}, \boldsymbol{D}_{0}^{*}, \boldsymbol{E}_{0}\right)=\inf _{\boldsymbol{F}} \inf _{\boldsymbol{D}_{0}}\left\{e\left(\boldsymbol{F}, \boldsymbol{D}_{0}\right)-\boldsymbol{E}_{0} \cdot \boldsymbol{D}_{0}\right\}
$$

where $\boldsymbol{E}_{0}$ is the externally controlled electric field. Thus, the stationary conditions of above functional arise as

$$
\boldsymbol{\mathcal { R }}_{\boldsymbol{F}}\left(\boldsymbol{F}, \boldsymbol{D}_{0}\right)=\partial_{\boldsymbol{F}} e=\mathbf{0} ; \quad \boldsymbol{\mathcal { R }}_{\boldsymbol{D}_{\mathbf{0}}}\left(\boldsymbol{F}, \boldsymbol{D}_{0}\right)=\partial_{\boldsymbol{D}_{0}} e-\boldsymbol{E}_{0}=\mathbf{0}
$$

The above nonlinear stationary conditions (104) are solved in terms of unknowns $\boldsymbol{F}^{*}$ and $\boldsymbol{D}_{0}^{*}$ dependent upon the externally controlled electric field $\boldsymbol{E}_{0}$. Similar set-up has been previously used in many references [26, 28], albeit restricted to the condition of plane strain and strict incompressibility. Here, these kinematic assumptions are relaxed and the deformation gradient tensor is left to adopt a more complex expression. Specifically, $\boldsymbol{F}$ and $\boldsymbol{E}_{0}$ are formulated as

$$
\boldsymbol{F}=\left[\begin{array}{ccc}
F_{11} & 0 & F_{13} \\
0 & F_{22} & F_{23} \\
0 & 0 & F_{33}
\end{array}\right] ; \quad \boldsymbol{E}_{0}=\left[\begin{array}{c}
0 \\
0 \\
E_{0}
\end{array}\right]
$$

where $F_{11}$ and $F_{22}$ represent in-plane stretching; $F_{13}$ and $F_{23}$, possible shearing effects; and $F_{33}$, the out-of-plane stretching. Typically, shearing components are disregarded and $F_{33}$ is constrained through the condition of strict incompressibility, as this permits to obtain closed-form solutions to the problem. Notice that these simplifying assumptions do not apply to this study, where the nonlinear stationary conditions (104) are solved by an iterative Newton-Raphson method. In addition, in order to track the entire equilibrium path beyond the onset of limit points (i.e., snap-through, snap-back), an arc-length technique is employed.

Two very distinct rank-one DE laminated composite materials are explored, whose properties are displayed in Table 1 and 2, respectively. For each of these materials, the contrast (i.e., ratio between inclusion and matrix) of material constituents is defined by parameters $f_{m}=\mu_{1}^{b} / \mu_{1}^{a}$ and $f_{e}=\epsilon_{b} / \epsilon_{a}$. Material 1 has mechanical contrast $f_{m}=3.9$ and electrical contrast $f_{e}=1$, whereas material 2 contrast values are $f_{m}=66$ and $f_{e}=31,250$. The microscale constituents of composite material 1 have properties resembling those of the well-known VHB-4910, whilst material 2 microscale constituents' properties match those of polyurethane and polyaniline for the soft and stiff phases, respectively (as used in [18]). Both composite materials are modelled as nearly incompressible.

First, the equilibrium paths are tracked for both materials 1 and 2 when varying the orientation of the laminate $\{\bar{\alpha}, \bar{\beta}\}$ as follows: $\bar{\alpha}=0^{\circ}$ is kept fixed and $\bar{\beta}$ is left to vary. The equilibrium paths are displayed in Figures 5 and 6 , where the Lagrangian electric field component $E_{0}$ is represented against the components $F_{11}$ and $F_{13}$ of the deformation gradient tensor (top row) and the norm of the deformation gradient and electric displacement amplitude vectors $\|\boldsymbol{\alpha}\|$ and $\|\boldsymbol{\beta}\|$, respectively (bottom row). Note that, for visualisation purposes, the electric field is normalised by $\sqrt{\bar{\mu} / \bar{\epsilon}}$, 


\begin{tabular}{llcccl}
\hline Properties & \multicolumn{2}{l}{ Phase $a$} & \multicolumn{2}{l}{ Phase $b$} & Units \\
\hline Mechanical parameters & $\mu_{1}^{a}$ & $1.0 \times 10^{5}$ & $\mu_{1}^{b}$ & $3.9 \times 10^{5}$ & $\mathrm{~Pa}$ \\
& $\mu_{2}^{a}$ & 0 & $\mu_{2}^{b}$ & 0 & $\mathrm{~Pa}$ \\
& $\lambda^{a}$ & $1.0 \times 10^{8}$ & $\lambda^{b}$ & $3.9 \times 10^{8}$ & $\mathrm{~Pa}$ \\
Electrical parameters & $\epsilon_{r}^{a}$ & 4 & $\epsilon_{r}^{b}$ & 4 & - \\
Concentration & $c^{a}$ & 0.5 & $c^{b}$ & 0.5 & - \\
\hline
\end{tabular}

Table 1: Numerical example 1. Material 1. Parameters of the model for each phase (see (27), (18)).

\begin{tabular}{llclcl}
\hline Properties & \multicolumn{2}{l}{ Phase $a$} & \multicolumn{2}{l}{ Phase $b$} & Units \\
\hline Mechanical parameters & $\mu_{1}^{a}$ & $1.0 \times 10^{7}$ & $\mu_{1}^{b}$ & $6.6 \times 10^{8}$ & $\mathrm{~Pa}$ \\
& $\mu_{2}^{a}$ & 0 & $\mu_{2}^{b}$ & 0 & $\mathrm{~Pa}$ \\
& $\lambda^{a}$ & $1.0 \times 10^{10}$ & $\lambda^{b}$ & $6.6 \times 10^{11}$ & $\mathrm{~Pa}$ \\
Electrical parameters & $\epsilon_{r}^{a}$ & 8 & $\epsilon_{r}^{b}$ & $2.5 \times 10^{5}$ & - \\
Concentration & $c^{a}$ & 0.5 & $c^{b}$ & 0.5 & - \\
\hline
\end{tabular}

Table 2: Numerical example 1. Material 2. Parameters of the model for each phase (see (27), (18)).

with $\bar{\mu}$ and $\bar{\epsilon}$ defined as the weighted arithmetic average of the individual microscale components, that is,

$$
\bar{\mu}=c^{a} \mu_{1}^{a}+c^{b} \mu_{1}^{b} ; \quad \bar{\epsilon}=\epsilon_{0}\left(c^{a} \epsilon_{r}^{a}+c^{b} \epsilon_{r}^{b}\right) .
$$

In general, all equilibrium paths are very nonlinear, exhibiting very distinct limit points, where the need for an arc-length technique is clearly justified. As it can be seen, for both material combinations, the component of orientation $\bar{\beta}$ has an important effect on both the shape of the equilibrium path and the development of the microscale amplitude vectors. In addition, by comparing Figure 5 (material 1) against Figure 6 (material 2), it is also clear that the properties of the microscale constituents have a drastic effect on the overall performance, for the same orientation of the laminate. It is also interesting to remark that, due to the inter-crossing of curves for different laminate orientations, it is not possible to naively predict what laminate orientation gives a better electro-mechanical performance, since this is strongly linked to the level of electric field applied. Thus, the optimum composite combination is dependent upon the operational range, both in terms of deformations and electric field.

Regarding material 1, and referring to Figure 5 top left, all laminate orientations undergo a buckling instability around $F_{11} \approx[1.2-1.4]$; however, the post-instability simulation enables to reach considerably higher values of around $F_{11} \approx 3.9(\approx 200 \%$ higher $)$. This shows that limiting the design of these components to the pre-buckling regime would imply a much lower electromechanical performance, hence the importance of harnessing the post-instability regime. In any case, positive values of $F_{11}$ (i.e., extension) are always observed. Referring to Figure 5 top right, the differences between the various laminate orientations are particularly evident. As an example, for the case $\bar{\beta}=89^{\circ}$, the equilibrium path displays a snap-back behaviour, where the shearing component $F_{13}$ changes from negative to positive values in a loop-like manner.

Regarding material 2, Figure 6 top left shows an important difference with respect to that of material 1 , namely $F_{11}$ takes negative values in the pre-instability region. A snap-back behaviour can be observed for orientations defined by $\bar{\beta}=24^{\circ}$ and $\bar{\beta}=32^{\circ}$. Similarly, Figure 6 top right depicts a very different response to that of material 1 , with a well-defined and smooth nonlinear equilibrium path given by values of $F_{13}$ always in the positive range.

Next, our attention shifts to the study of the effect that the microstructure has on the propa- 

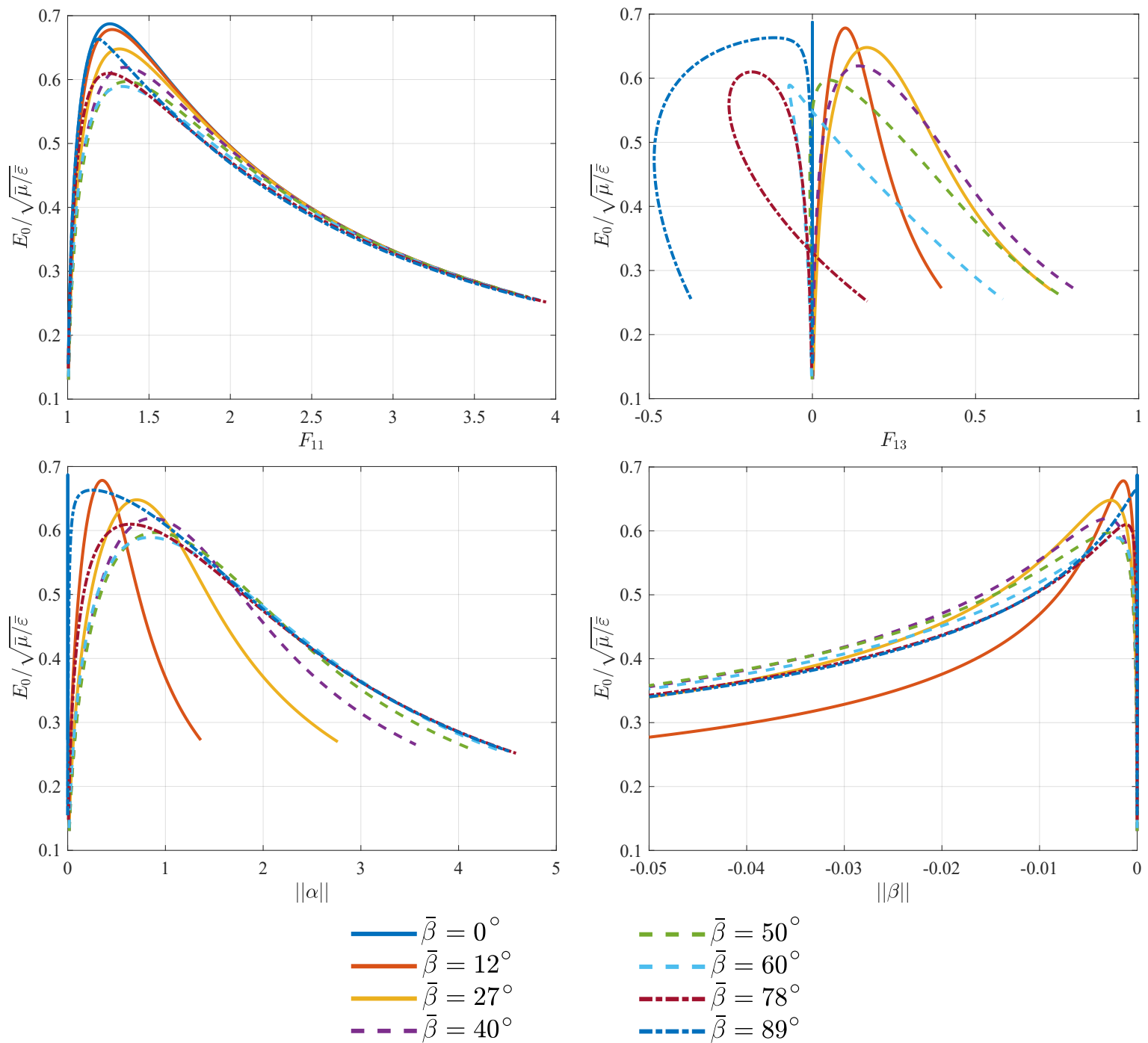

$$
\begin{aligned}
& --\bar{\beta}=50^{\circ} \\
& ---\bar{\beta}=60^{\circ} \\
& --\backsim-\bar{\beta}=78^{\circ} \\
& -\backsim-\bar{\beta}=89^{\circ}
\end{aligned}
$$

Figure 5: Numerical example 1. Material 1. Equilibrium path curves for the case $\bar{\alpha}=0^{\circ}$. For each material there are 4 graphs, which represent, for various values of angle $\bar{\beta}$, the electric field (normalised with $\bar{\mu}, \bar{\epsilon}$ defined in (109)) against: (top-left) the component $F_{11}$ of the deformation gradient tensor $\boldsymbol{F}$, (top-right) the component $F_{13}$, (bottom-left) the magnitude $\|\boldsymbol{\alpha}\|$ of the mechanical amplitude vector $\boldsymbol{\alpha}$, (bottom-right) the magnitude $\|\boldsymbol{\beta}\|$ of the electrical amplitude vector $\boldsymbol{\beta}$. 

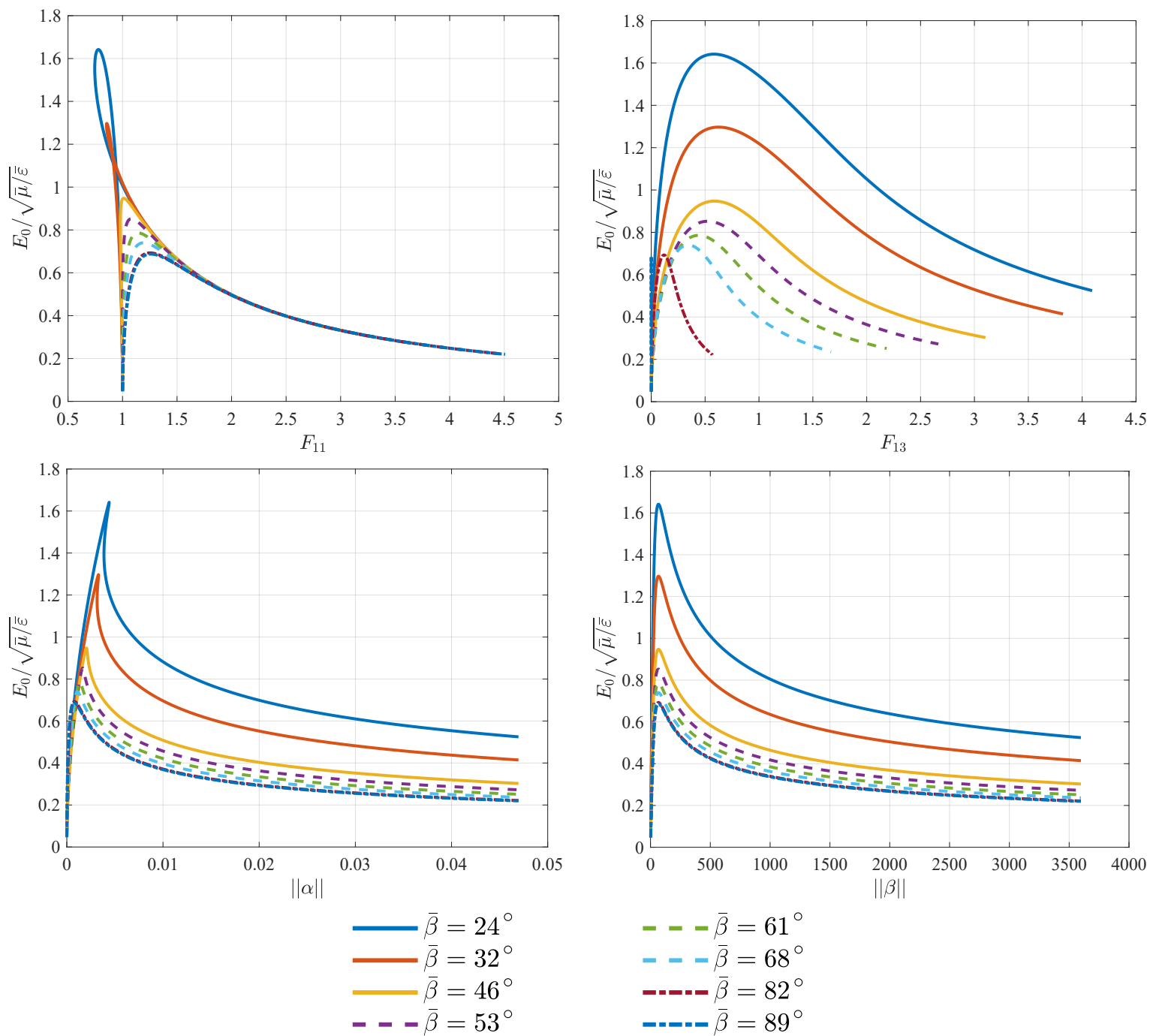

$$
\begin{aligned}
& --\bar{\beta}=61^{\circ} \\
& ---\bar{\beta}=68^{\circ} \\
& ---\bar{\beta}=82^{\circ} \\
& --\square-\bar{\beta}=89^{\circ}
\end{aligned}
$$

Figure 6: Numerical example 1. Material 2. Equilibrium path curves for the case $\bar{\alpha}=0^{\circ}$. For each material there are 4 graphs, which represent, for various values of angle $\bar{\beta}$, the electric field (normalised with $\bar{\mu}, \bar{\epsilon}$ defined in (106)) against: (top-left) the component $F_{11}$ of the deformation gradient tensor $\boldsymbol{F}$, (top-right) the component $F_{13}$, (bottom-left) the magnitude $\|\boldsymbol{\alpha}\|$ of the mechanical amplitude vector $\boldsymbol{\alpha}$, (bottom-right) the magnitude $\|\boldsymbol{\beta}\|$ of the electrical amplitude vector $\boldsymbol{\beta}$. 
gation of shear wave speeds within the composite. Notice that volumetric wave speeds are not of interest in this case due to the nearly incompressible nature of the constituents. A similar study was carried out in $[25,26,66]$, albeit restricted to the case of isotropic in-plane stretching and strict incompressibility, where only component $F_{11}$ was regarded as unknown. In this work, we are not restricted by this kinematic assumption. In general, for the case of a plane wave propagating within a continuum in a direction defined by the unit vector $\boldsymbol{\nu}$, the electro-mechanical acoustic tensor $\boldsymbol{Q}$ can be found as [52]

$$
\boldsymbol{Q}=\mathcal{C}_{\boldsymbol{\nu} \boldsymbol{\nu}}-\mathcal{Q}_{\nu}^{T} \boldsymbol{\theta}^{-1}\left(\boldsymbol{I}-\frac{\left(\boldsymbol{\nu} \otimes \boldsymbol{\theta}^{-1} \boldsymbol{\nu}\right)}{\boldsymbol{\nu} \cdot \boldsymbol{\theta}^{-1} \boldsymbol{\nu}}\right) \mathcal{Q}_{\boldsymbol{\nu}}
$$

where

$$
\left(\mathcal{C}_{\nu \nu}\right)_{i j}=\mathcal{C}_{i I j J} \nu_{I} \nu_{J}, \quad\left(\mathcal{Q}_{\nu}\right)_{I j}=\mathcal{Q}_{I j J} \nu_{J}
$$

The squared root of the eigenvalues of the acoustic tensor provides the values of the volumetric and shear wave speeds. We first restrict ourselves to the case of plane waves propagating along directions defined by a unit vector $\boldsymbol{\nu}$ contained within the plane $O X_{1} X_{2}$ (i.e., $\boldsymbol{\nu} \cdot[0,0,1]^{T}=0$ ), and compute the inverse of the Euclidean norm of the shear wave speeds (i.e., slowness). A polar representation of the slowness is shown in Figures 7 and 8 for materials 1 and 2, respectively. In both Figures 7 and 8, each of the nine sub-Figures included depicts slowness curves for a given orientation of the laminate. Specifically, the angle $\bar{\alpha}=0^{\circ}$ is kept fixed, and the angle $\bar{\beta}$ takes the values indicated in the legend below the figures. Within each subfigure, six different slowness curves are displayed for increasing values of the norm of the electric displacement vector $\left\|\boldsymbol{D}_{0}\right\|$, which is in turn normalised by $\sqrt{\tilde{\mu} \tilde{\epsilon}}$, with $\tilde{\mu}$ and $\tilde{\epsilon}$ defined as the weighted harmonic average of the individual microscale components, that is,

$$
\tilde{\mu}=\left(\frac{c^{a}}{\mu_{1}^{a}}+\frac{c^{b}}{\mu_{1}^{b}}\right)^{-1} ; \quad \tilde{\epsilon}=\epsilon_{0}\left(\frac{c^{a}}{\epsilon_{r}^{a}}+\frac{c^{b}}{\epsilon_{r}^{b}}\right)^{-1} .
$$

It is clear that laminate orientation, electric displacement operational range and microscale materials composition have a dramatic impact upon the shape of the slowness curves. It is observed that, as the operational range increases, the slowness curves lose their initial circular shape to adopt a more anisotropic pattern. This evolution develops smoothly in material 1 (see Figure 7 ) and is more pronounced for material 2 (see Figure 8). Moreover, patterns are not necessarily concentric between different operational ranges, which highlights the strong nonlinearity of the electro-mechanical coupling effect and its impact upon the propagation of shear waves.

Next, in Figure 8 the slowness curves for material 2 on the same plane $O X_{1} X_{2}$ are illustrated. In this case, it is observed that the electric displacement field has a big impact on the wave propagation curves, for all values of angle $\bar{\beta}$. For small values of the angle $\bar{\beta}$, there is a big anisotropy for large values of electric displacement field, whilst the behaviour remains more isotropic for small values of it. For intermediate values of $\bar{\beta}$, some curves widen along the $X_{1}$ direction, whilst for larger values of $\bar{\beta}$, they tend to adopt a more isotropic behaviour. As a conclusion, both the electric displacement field and the microscale do affect the wave propagation speed, and it is more noticeable on material 2, since the contrast between mechanical and electrical properties is larger.

Finally, we generalise the study previously presented, analysing the evolution of the acoustic tensor $\boldsymbol{Q}$ (107) for any three dimensional arbitrary orientation $\boldsymbol{\nu}$ as a function of the deformation process for the case of a specific laminate configuration. To aid the visualisation of this tensor, we restrict ourselves to the monitoring of the smallest of its minors, and we display it in spherical coordinates, that is, we plot vector $\boldsymbol{q}$, defined as

$$
\boldsymbol{q}=q \boldsymbol{\nu} ; \quad q=\min \left(\frac{Q_{11}}{\bar{\mu}}, \frac{Q_{11} Q_{22}-Q_{12} Q_{21}}{\bar{\mu}^{2}}, \frac{\operatorname{det} \boldsymbol{Q}}{\bar{\mu}^{3}}\right),
$$



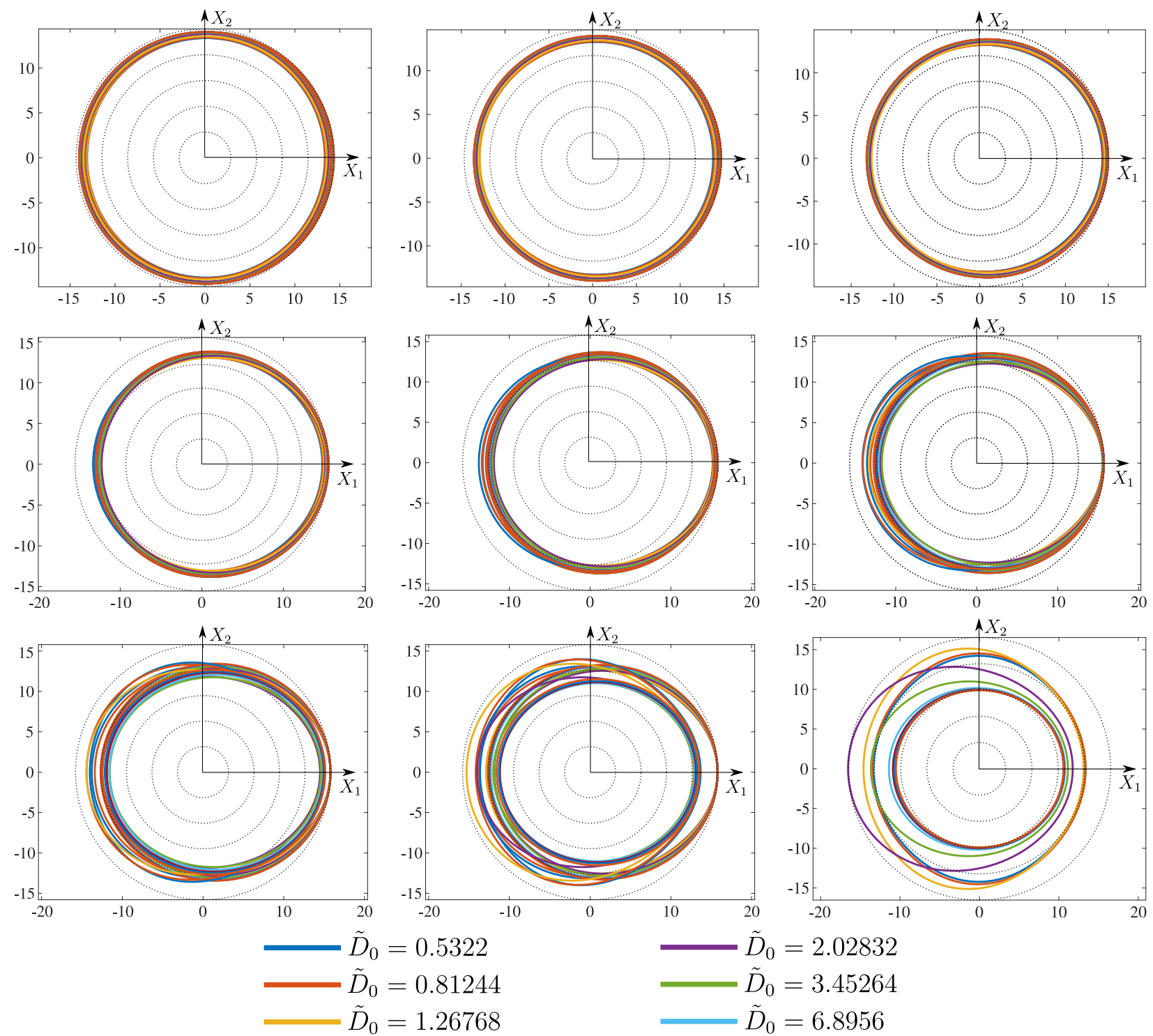

Figure 7: Numerical example 1. Material 1. Slowness curves $\left(\frac{c_{s}}{\bar{\mu}}\right)^{-1}$ for $\bar{\alpha}=0^{\circ}$ and, from left to right and from top to bottom, $\bar{\beta}=\left\{0^{\circ}, 9^{\circ}, 18^{\circ}, 30^{\circ}, 42^{\circ}, 54^{\circ}, 66^{\circ}, 78^{\circ}, 89^{\circ}\right\}$, for the various values of normalised electric displacement field $\tilde{D}_{0}=\left\|\frac{D_{0}}{\sqrt{\tilde{\mu} \tilde{\epsilon}}}\right\|$ indicated in the legend below the Figures. For visualisation purposes, a dotted circular grid is added in each picture. 

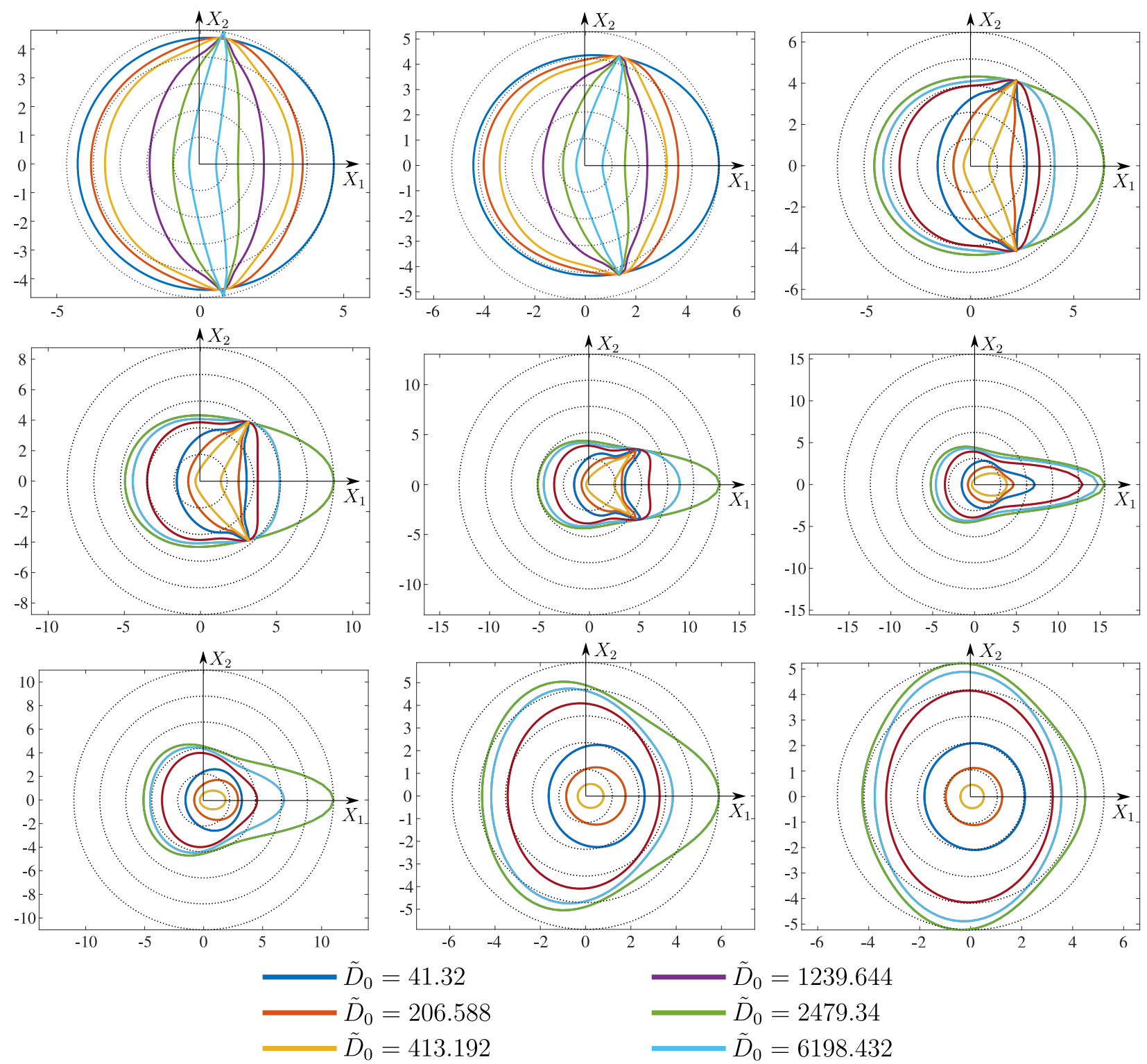

Figure 8: Numerical example 1. Material 2. Slowness curves $\left(\frac{c_{s}}{\bar{\mu}}\right)^{-1}$ for $\bar{\alpha}=0^{\circ}$ and, from left to right and from top to bottom, $\bar{\beta}=\left\{10^{\circ}, 17.5^{\circ}, 24^{\circ}, 32^{\circ}, 40^{\circ}, 46.5^{\circ}, 54^{\circ}, 68^{\circ}, 83^{\circ}\right\}$, for the various values of normalised Lagrangian electric displacement field $\tilde{D}_{0}=\left\|\frac{D_{0}}{\sqrt{\tilde{\mu} \tilde{\epsilon}}}\right\|$ indicated in the legend below the Figures. For visualisation purposes, a dotted circular grid is added in each picture. 
where $\bar{\mu}$ is given by (106)a.

In Figure 9, vector $\boldsymbol{q}$ (110) for every orientation $\boldsymbol{\nu}$ is represented at three specific deformation states along the curve of normalised electric field against the deformation component $F_{11}$, for material 2 with laminate orientation defined by $\bar{\alpha}=0^{\circ}$ and $\bar{\beta}=82^{\circ}$. It can be inferred that the acoustic tensor is strongly dependent on the deformation and electric field, being its orientation and shape different in the three states represented. In addition, its highly anisotropy is remarked, since its shape differs significantly from that corresponding to an isotropic state (i.e., a sphere). The values adopted in the colour bars are a clear indication that the minors of $\boldsymbol{Q}$ remain nonnegative for the entire deformation process, ensuring macroscopic stability, that is, ellipticity of the homogenised tangent operator.

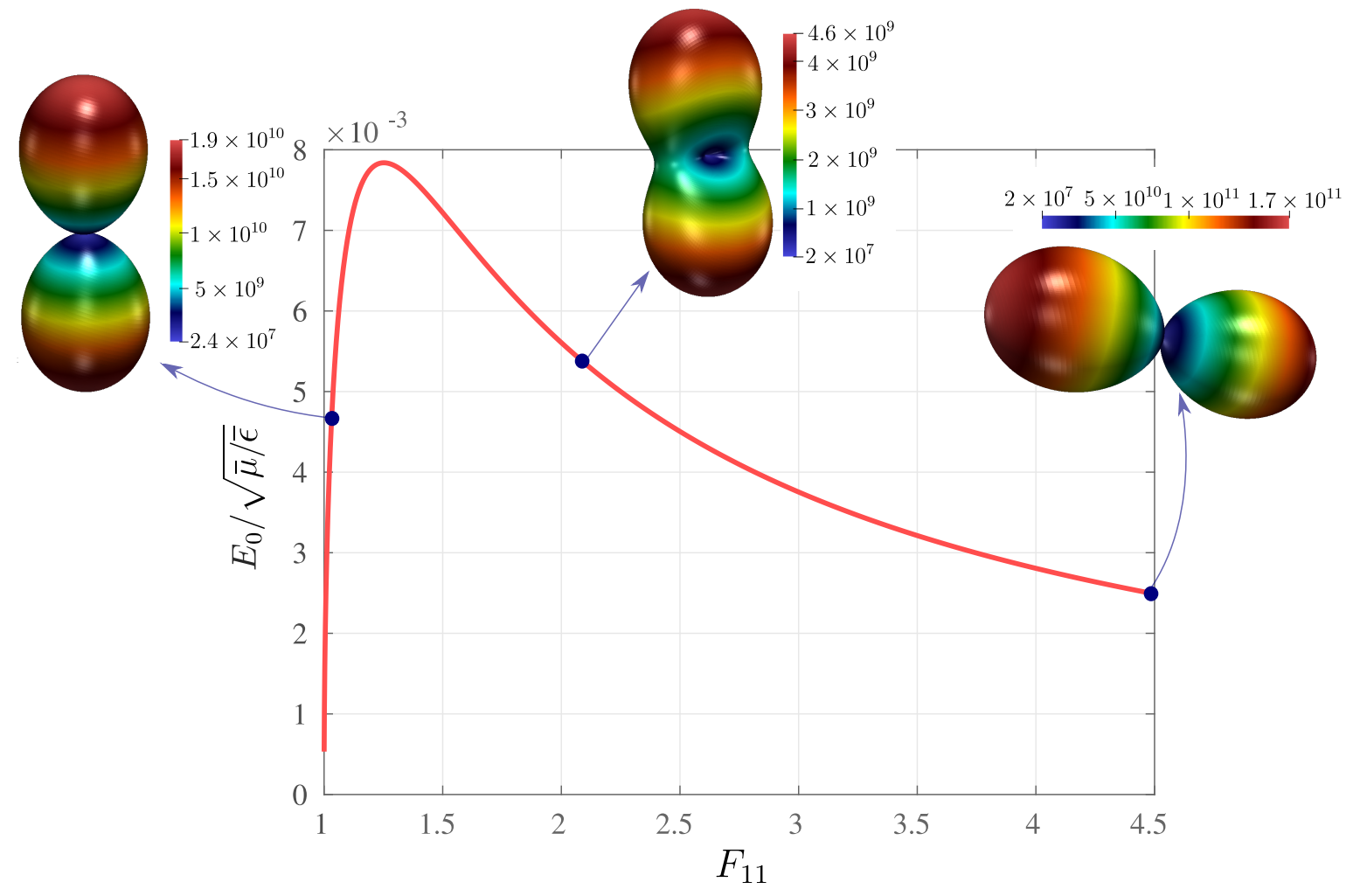

Figure 9: Numerical example 1. Material 2. Evolution of spherical parametrisation of the least of the minors of the electro-mechanical acoustic tensor at three particular states of the curve of normalised electric field against deformation gradient tensor component $F_{11}$. The orientation of the laminate is given by $\bar{\alpha}=0^{\circ}$ and $\bar{\beta}=82^{\circ}$.

\subsection{Numerical example 2: effect of concentration and non-CMV energy contributions}

Through this example we aim to:

- Study the effect that the composite concentration has in the macroscopic ellipticity of the laminate when considering high electrical and mechanical contrasts.

- Compare the response of two laminated composite models, one made of pure CMV phases and one where the CMV phases are slightly perturbed through the addition of a non-CMV energy contribution.

The set-up (i.e. geometry and boundary conditions) of the numerical example is the same as that used in the previous example, depicted in Figure 4. The material properties for the composite phases are displayed in Table 3, and have been chosen identical to those used for material 2 in the previous example, yielding contrasts $f_{m}=66$ and $f_{e}=31,250$. 


\begin{tabular}{llcccl}
\hline Properties & \multicolumn{2}{l}{ Phase $a$} & \multicolumn{2}{l}{ Phase $b$} & Units \\
\hline Mechanical parameters & $\mu_{1}^{a}$ & $1.0 \times 10^{7}$ & $\mu_{1}^{b}$ & $6.6 \times 10^{8}$ & $\mathrm{~Pa}$ \\
& $\mu_{2}^{a}$ & 0 & $\mu_{2}^{b}$ & 0 & $\mathrm{~Pa}$ \\
& $\lambda^{a}$ & $1.0 \times 10^{10}$ & $\lambda^{b}$ & $6.6 \times 10^{11}$ & $\mathrm{~Pa}$ \\
Electrical parameters & $\epsilon_{r}^{a}$ & 8 & $\epsilon_{r}^{b}$ & $2.5 \times 10^{5}$ & - \\
Concentration & $c^{a}$ & {$[0.5,0.99]$} & $c^{b}$ & {$[0.5,0.01]$} & - \\
\hline
\end{tabular}

Table 3: Numerical example 2. Parameters of the model for each phase (see (27), (18))

With regards to the first objective of the example, a first study is focused on the effect that the concentration of the composite constituents may have upon the loss of macroscopic ellipticity, since it has been reported in the literature [30] that for materials with high contrast $f_{m}$, loss of macroscopic ellipticity may occur when the concentration of the stiff phase is relatively high. Only one particular orientation (corresponding to $\bar{\alpha}=0^{\circ}, \bar{\beta}=82^{\circ}$ ) is presented in the results, although similar conclusions have been obtained for other configurations.

Two concentrations are considered: $c^{b}=0.5$ (high concentration of the stiff phase) and $c^{b}=0.01$ (low concentration of the stiff phase). Due to the high contrast between the phases, the averaged properties of the composite material approach (in order of magnitude) those of the stiff phase, namely $\bar{\mu}=c^{a} \mu^{a}+c^{b} \mu^{b} \approx c^{b} \mu^{b}, \bar{\epsilon}=c^{a} \epsilon^{a}+c^{b} \epsilon^{b} \approx c^{b} \epsilon^{b}$. In Figures 10(a),(b), the Lagrangian electric field component $E_{0}$ (normalised by $\sqrt{\bar{\mu} / \bar{\epsilon}}$ as defined in (106)) is represented against the component $F_{11}$ of the deformation gradient tensor. The continuous blue line represents the equilibrium path (satisfaction of (104)), whilst the red regions indicate the possible areas of loss of macroscopic ellipticity. A similar study can be found in [58]. In Figures 10(a),(b), the equilibrium path lies away from the regions of loss of macroscopic ellipticity, but the effect of the concentration can be clearly observed. Indeed, Figure 10(a) shows that when the concentration of the stiff phase is high $c^{b}=0.5$, the material can potentially show loss of macroscopic stability in two scenarios; first, for a small decrease of the electric field (for the entire range of deformation $F_{11}$ shown in the figure) and second, for a very large increase of the electric field (for values of $F_{11}$ in the range from 1.6 to 1.8). On the contrary, when the concentration of the stiff phase is small $\left(c^{b}=0.01\right)$, then the loss of macroscopic stability can only be observed for large decreases in the electric field.

Regarding the second objective of this example, let us consider the following electromechanical internal energy contribution for each of the laminate phases $i=a, b$,

$$
W_{e m}^{i}\left(\boldsymbol{F}, J, \boldsymbol{D}_{0}, \boldsymbol{d}\right)=\underbrace{\frac{I I_{\boldsymbol{d}}}{2 \epsilon_{r}^{i} \epsilon_{0} J}}_{\text {CMV contr. }}+\gamma^{i}(\underbrace{\frac{I I_{\boldsymbol{d}}}{2 \epsilon_{r}^{i} \epsilon_{0} J}}_{\text {CMV contr. }}-\underbrace{\frac{I I_{\boldsymbol{D}_{0} I I_{\boldsymbol{F}}}}{6 \epsilon_{r}^{i} \epsilon_{0}}}_{\text {Non-CMV contr. }}) ; \quad i=\{a, b\},
$$

where a CMV energy contribution is perturbed via the addition of a non-CMV energy contribution, with $\gamma^{i}$ a positive dimensionless parameter close to zero. In what follows, $\gamma^{a}=\gamma^{b}$ will be considered. As can be expected, the $\gamma^{i}$-term induces a perturbation of the pure CMV energy, albeit carefully designed to yield a similar electromechanical response (in terms of the dielectric tensor near the origin) to the pure CMV model. Indeed,

$$
\left.\boldsymbol{\theta}^{i}\right|_{\boldsymbol{F = I}, \boldsymbol{D}_{0}=0}=\left.\partial_{\boldsymbol{D}_{0} \boldsymbol{D}_{0}}^{2} W_{e m}^{i}\right|_{\boldsymbol{F}=\boldsymbol{I}, \boldsymbol{D}_{0}=0}=\frac{1}{\epsilon_{r}^{i} \epsilon_{0}} \boldsymbol{I}+\gamma^{i}\left(\frac{1}{\epsilon_{r}^{i} \epsilon_{0}} \boldsymbol{I}-\frac{1}{\epsilon_{r}^{i} \epsilon_{0}} \boldsymbol{I}\right)=\frac{1}{\epsilon_{r}^{i} \epsilon_{0}} \boldsymbol{I} .
$$

Figure 11 displays similar results to those in previous Figure 10, namely, equilibrium path in blue and regions of loss of macroscopic stability in red, for different values of the coefficient $\gamma^{a}=\gamma^{b}$. 


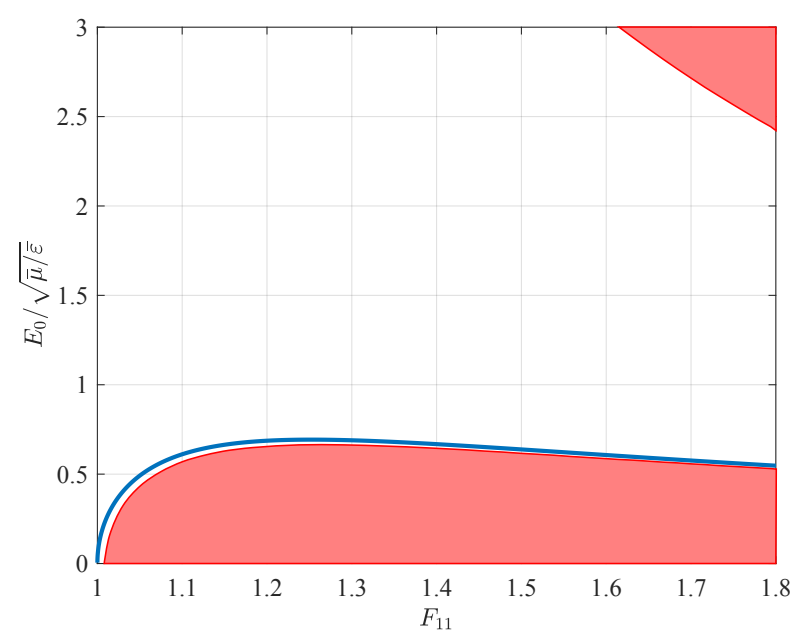

(a)

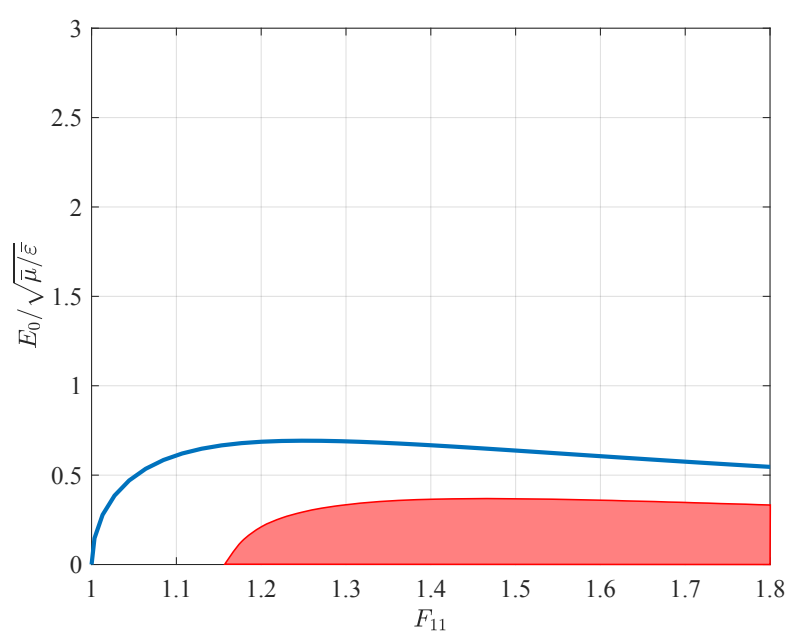

(b)

Figure 10: Numerical example 2: Study of loss of macroscopic ellipticity for two concentrations of the composite material: (a) $c^{b}=0.5$, (b) $c^{b}=0.01$. The normalised electric field is represented against the component $F_{11}$ of the deformation gradient tensor $\boldsymbol{F}$. The equilibrium path curve for the case $\bar{\alpha}=0^{\circ}, \bar{\beta}=82^{\circ}$ is represented with a continuous blue line, whilst the shadow red regions represent the areas involving loss of macroscopic ellipticity.

As can be observed, even small values of the parameter $\gamma^{i}$, whilst respecting very faithfully the equilibrium path of the pure CMV based model, can induce larger regions of possible loss of macroscopic stability. The higher the value of $\gamma^{i}$ is, the larger the area of loss of macroscopic ellipticity is. Crucially, the value of $\gamma^{i}$ has been kept small to ensure similar equilibrium paths between the pure CMV model and the model including non-CMV contributions, permitting a fair comparison. When $\gamma^{i}$ is increased drastically, our studies show that the equilibrium path changes drastically (it is not comparable any more) and, moreover, solution to the microscopic problem recast in (69) cannot always be obtained due to lack of convergence.

\subsection{Numerical example 3: complex deformation of a rectangular multi-layered DE film}

In this example we aim to:

- Capture the various deformation modes that a rank-one DE laminated composite, in the form of a rectangular film, can undergo in response to electric stimuli.

- Compare the response of the composite for different orientations of the laminate and provide some simple strain metrics to distinguish actuation deformation modes.

- Analyse the possible local loss of macroscopic ellipticity in order to ensure that spurious mesh dependent effects are not developed in the Finite Element analysis.

We consider a rectangular DE rank-one laminate film of dimensions $0.1 \mathrm{~m} \times 0.03 \mathrm{~m} \times 0.001 \mathrm{~m}$, fully clamped at face $X_{1}=0$ and free everywhere else, as depicted in Figure 12. Two electrodes are placed at the bottom and mid surfaces of the specimen and a voltage difference of $\Delta V=2 \times 10^{4}$ $\mathrm{V}$ is applied across. The geometrical and simulation parameters for this example are collected in Table 4 . The domain is discretised using a $80 \times 20 \times 2$ hexahedral structured mesh, with $Q 2$ Finite Elements used to interpolate both displacement and electric potential, being 132, 020 the total number of degrees of freedom of the problem. The solver coupling strategy is monolithic, with a tolerance for the Newton-Raphson method of $10^{-6}$ in Euclidean norm. The voltage gradient is applied in 400 increments, being $\Lambda \in[0,1]$ the so-called load factor.

The constitutive models used to describe the rank-one laminate phases $a$ and $b$ are those of an ideal dielectric Mooney-Rivlin type of elastomer (refer to (27) and (18)), and the material properties used are displayed in Table 5. Notice that the Mooney-Rivlin parameters $\mu_{1}^{a, b}$ and 


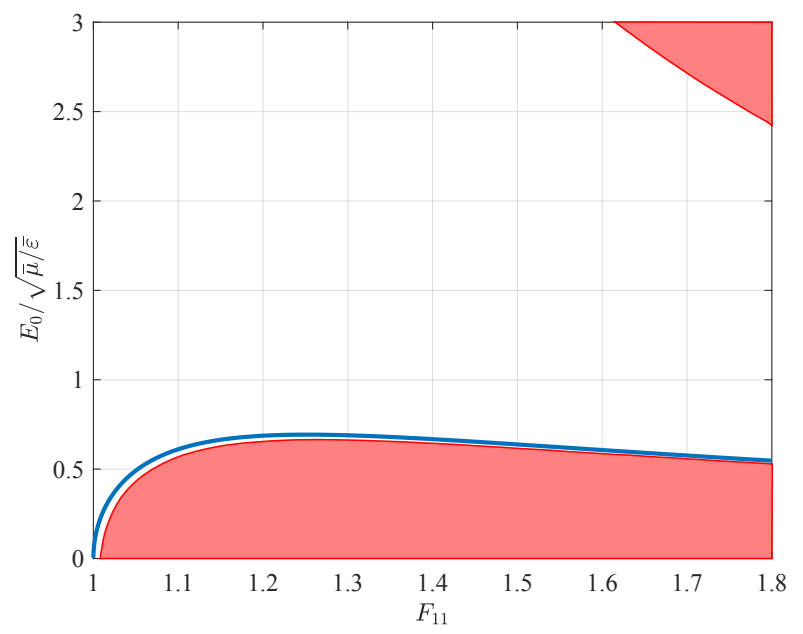

(a)

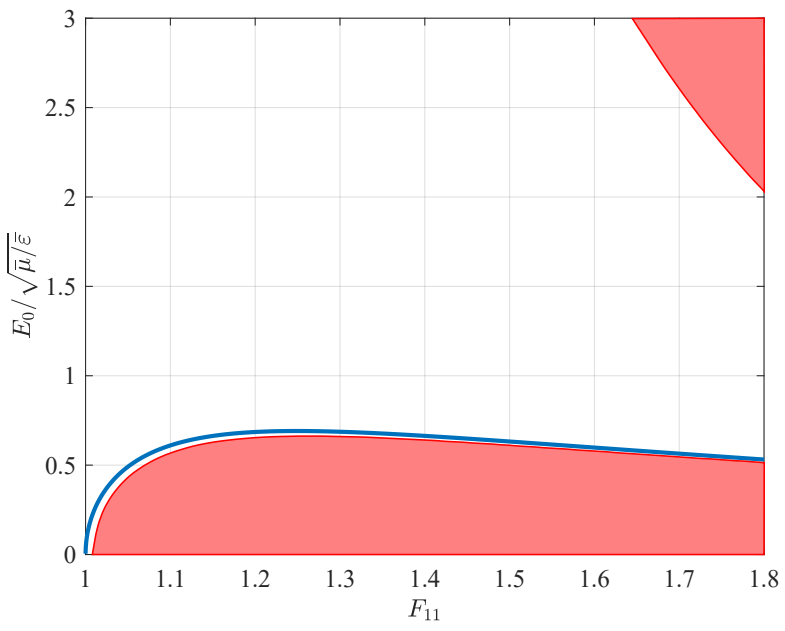

(b)

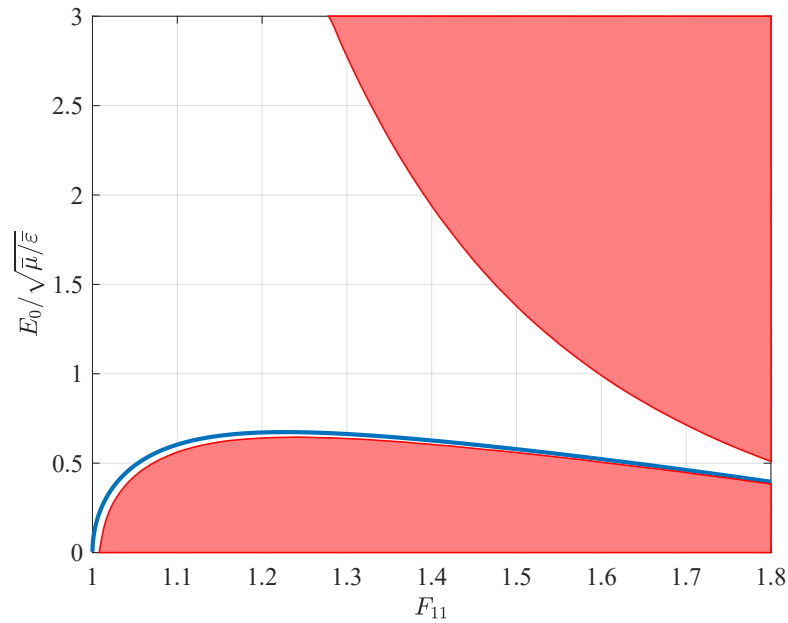

(c)

Figure 11: Numerical example 2: Study of loss of macroscopic ellipticity for: (a) model with CMV phases $\left(\gamma^{a}=\right.$ $\gamma^{b}=0$ in (111)), models with non-CMV phases: (b) $\gamma^{a}=\gamma^{b}=0.001$, (c) $\gamma^{a}=\gamma^{b}=0.01$. The normalised electric field is represented against the component $F_{11}$ of the deformation gradient tensor $\boldsymbol{F}$. The equilibrium path curve for each case is represented with a continuous blue line, whilst the red shadow areas indicate loss of macroscopic ellipticity. The orientation of the laminate considered is $\bar{\alpha}=0^{\circ}, \bar{\beta}=82^{\circ}$.

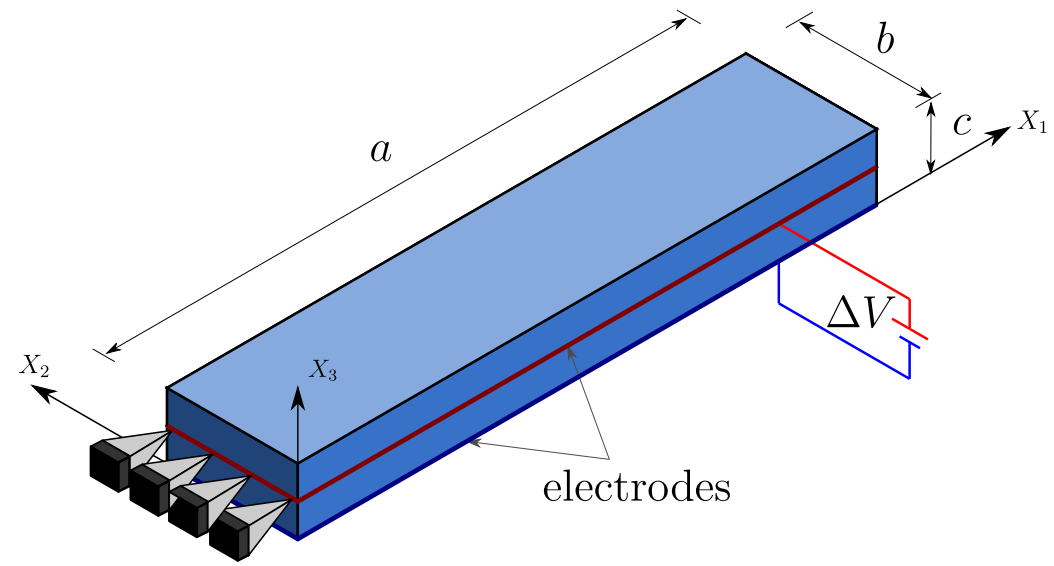

Figure 12: Numerical example 3. Geometry and boundary conditions.

$\mu_{2}^{a, b}$ can be used to define a unique shear moduli, in this case of values $\mu^{a}=10 \mathrm{MPa}$ (typical of the polyurethane $(\mathrm{PU})$ ) and $\mu^{b}=660 \mathrm{MPa}$ (typical of the polyaniline (PANI)) $[18,38]$. With these material properties, the mechanical and electrical contrasts of the composite are given by 


\begin{tabular}{lcclllc}
\hline Geometrical parameters & $a$ & 0.1 & $\mathrm{~m}$ & Simulation parameters & $N_{x}$ & 80 \\
& $b$ & 0.03 & $\mathrm{~m}$ & & $N_{y}$ & 20 \\
& $c$ & 0.001 & $\mathrm{~m}$ & & $N_{z}$ & 2 \\
Voltage applied & $\Delta V$ & 20 & $\mathrm{kV}$ & Newton tolerance & & $10^{-6}$ \\
\hline
\end{tabular}

Table 4: Numerical example 3. Geometrical and simulation parameters.

$f_{m}=\mu^{a} / \mu^{b}=66$ and $f_{e}=\epsilon_{r}^{a} / \epsilon_{r}^{b}=31,250$, respectively. These are the same contrast values used for material 2 in the previous example section.

\begin{tabular}{llclll}
\hline Properties & \multicolumn{2}{l}{ Phase $a$} & \multicolumn{2}{l}{ Phase $b$} & Units \\
\hline Mechanical parameters & $\mu_{1}^{a}$ & $8.5 \times 10^{6}$ & $\mu_{1}^{b}$ & $5.6 \times 10^{8}$ & $\mathrm{~Pa}$ \\
& $\mu_{2}^{a}$ & $1.5 \times 10^{6}$ & $\mu_{2}^{b}$ & $1.0 \times 10^{8}$ & $\mathrm{~Pa}$ \\
& $\lambda^{a}$ & $4.3 \times 10^{7}$ & $\lambda^{b}$ & $2.8 \times 10^{8}$ & $\mathrm{~Pa}$ \\
Electrical parameters & $\epsilon_{r}^{a}$ & 8 & $\epsilon_{r}^{b}$ & $2.5 \times 10^{5}$ & - \\
Concentration & $c^{a}$ & 0.95 & $c^{b}$ & 0.05 & - \\
\hline
\end{tabular}

Table 5: Numerical example 3. Material parameters of the model for each phase (see (27), (18)).

Figure 13 displays the evolution of the deformation for increasing values of the load factor $\Lambda$ and for various laminate orientations defined by angles $\{\bar{\alpha}, \bar{\beta}\}$. To emphasise the influence of the composite arrangement with respect to that of a single-phase material, Figure 13 (a) displays the response when the volume fraction is $c^{a}=1$, whereas Figures 13 (b)-(f) are for a constant volume fraction $c^{a}=0.95$ and multiple $\{\bar{\alpha}, \bar{\beta}\}$ laminate combinations, namely $\left\{0^{\circ}, 30^{\circ}\right\},\left\{0^{\circ}, 60^{\circ}\right\}$, $\left\{0^{\circ}, 90^{\circ}\right\},\left\{15^{\circ}, 90^{\circ}\right\}$ and $\left\{75^{\circ}, 90^{\circ}\right\}$. It is evident that the consideration of a rank-one laminate immediately introduces a considerably more sophisticated actuation mode, where the pure monoaxial bending response displayed in Figure 13 (a) is transformed into bi-axial bending with possible torsion development. This Figure clearly highlights the appeal for the use of rank-one laminates in order to access advanced actuation modes in soft robotics. Figures 13 (b)-(d) show an increase in bending about $\mathrm{OX}_{2}$ axis with respect to that displayed by the single-phase material, with development of bending about the perpendicular $O X_{1}$ axis. Interestingly, for non-zero values of $\bar{\alpha}$, the composite manifests high levels of torsion about $O X_{3}$ axis.

In order to provide a simple metric for comparison, we compute the normalised integral value of the Green-Lagrange strain tensor $\varepsilon$, defined as

$$
\boldsymbol{\varepsilon}=\frac{1}{|V|} \int_{V} \boldsymbol{E} d V ; \quad \boldsymbol{E}=\frac{1}{2}\left(\boldsymbol{F}^{T} \boldsymbol{F}-\boldsymbol{I}\right) .
$$

Figures 14 and 15 display the evolution of some of the components of $\varepsilon$ for increasing values of the load factor $\Lambda$ and for various laminate orientations defined by angles $\{\bar{\alpha}, \bar{\beta}\}$. Figure 14 displays components $\varepsilon_{11}$ and $\varepsilon_{22}$ when $\bar{\alpha}=0^{\circ}$ and $\bar{\beta}$ is left to vary. The curves are smooth with increasing values of $\varepsilon_{11}$ and $\varepsilon_{22}$ as a function of the load factor. As it can be observed, curves show a symmetry for laminate orientations with $\bar{\beta}$ above and below $90^{\circ}$. Figure 14 bottom clearly shows that higher values of $\varepsilon_{22}$ are related to the development of bi-axial bending, whereas lower values are more prone to display mono-axial bending. As for Figure 14 top, it is interesting to observe the change in sign of the component $\varepsilon_{11}$ for lamination angle at around $\bar{\beta} \approx 55^{\circ}$ (and its supplementary), emphasising the difficulty to predict a priori the response of the material without resorting to in silico simulation.

In Figure 15, similar curves are displayed for $\varepsilon_{11}$ and $\varepsilon_{23}$ for laminate orientations defined by $\bar{\beta}=10^{\circ}$ (top and middle diagrams), $\bar{\beta}=90^{\circ}$ (bottom diagram) and $\bar{\alpha}$ left to vary. Similar 


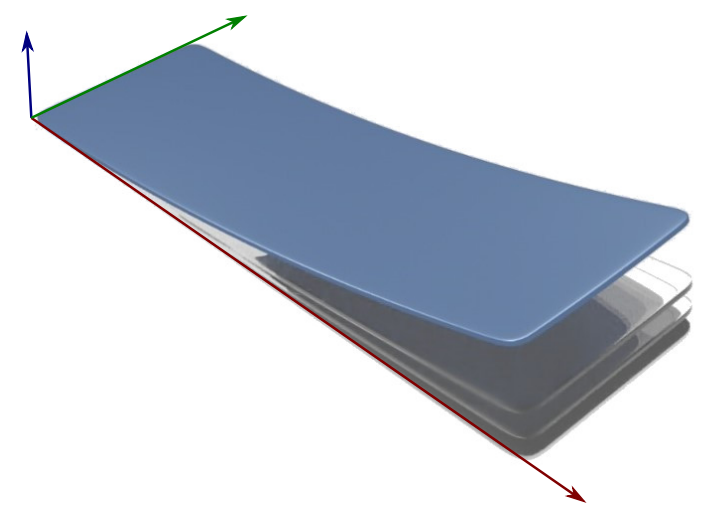

(a)

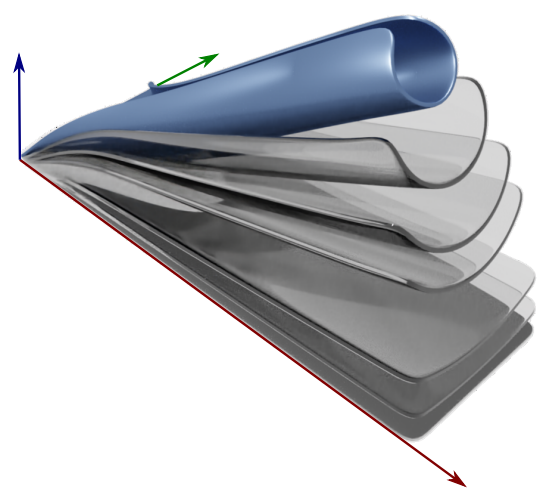

(c)

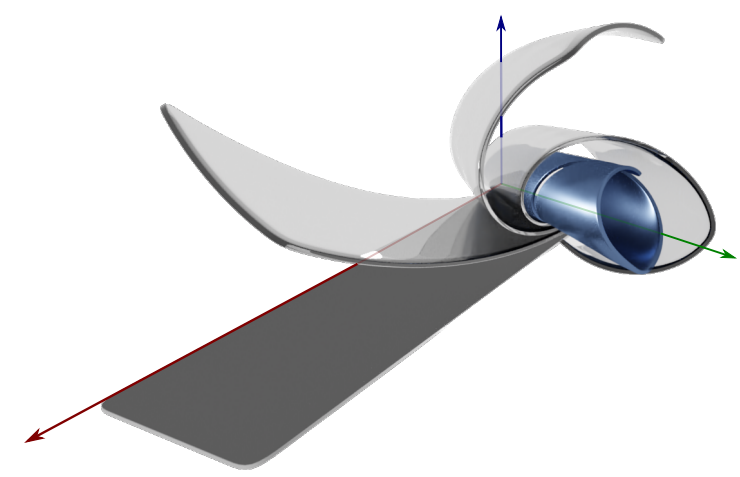

(e)

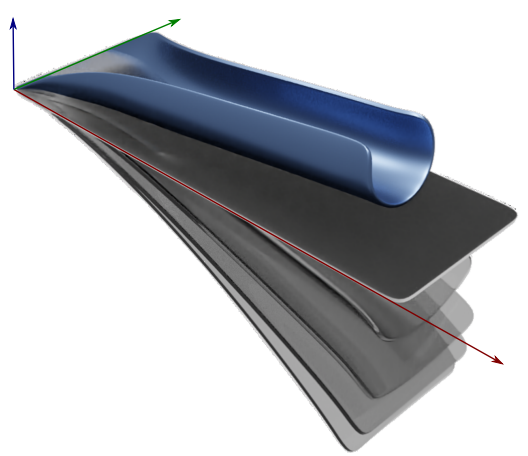

(b)

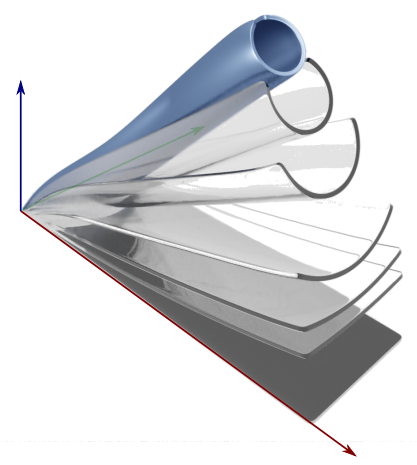

(d)

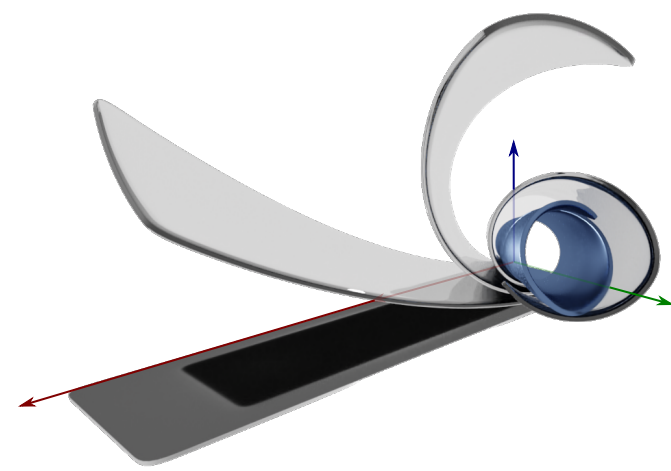

(f)

Figure 13: Numerical example 3. Deformation evolution in the cases (a) single-phase, (b) $\bar{\alpha}=0^{\circ}, \bar{\beta}=30^{\circ}$, (c) $\bar{\alpha}=0^{\circ}, \bar{\beta}=60^{\circ}$, (d) $\bar{\alpha}=0^{\circ}, \bar{\beta}=90^{\circ}$, (e) $\bar{\alpha}=15^{\circ}, \bar{\beta}=90^{\circ}$, (f) $\bar{\alpha}=75^{\circ}, \bar{\beta}=90^{\circ}$.

conclusions to those of the previous figure can be inferred here. Precisely, smooth curves are displayed with a symmetric (or skew-symmetric) pattern for angles $\bar{\alpha}$ above and below $90^{\circ}$. Interestingly, Figure 15 bottom displays an anti-symmetric pattern explained by the sign of the rotation (positive or negative) depending upon $\bar{\alpha}$ been above or below $90^{\circ}$. Once again, it is clear the design opportunities in terms of optimisation that the current computational framework can offer, where specific strain metrics (for instance) can be used in order to optimise the shape of the elastomer, its microstructure composition or the distribution of electrodes.

A study of the possible loss of macroscale ellipticity and convexity is conducted. For the sake of simplicity, we present the results for a specific orientation of the laminate and two states of deformation. To study loss of ellipticity, we compute the field distribution of the smallest of the minors (110) of the acoustic tensor for any possible orientation $\boldsymbol{\nu}$, namely

$$
\mathrm{I}_{\text {ellip }}=\min _{\nu} q
$$



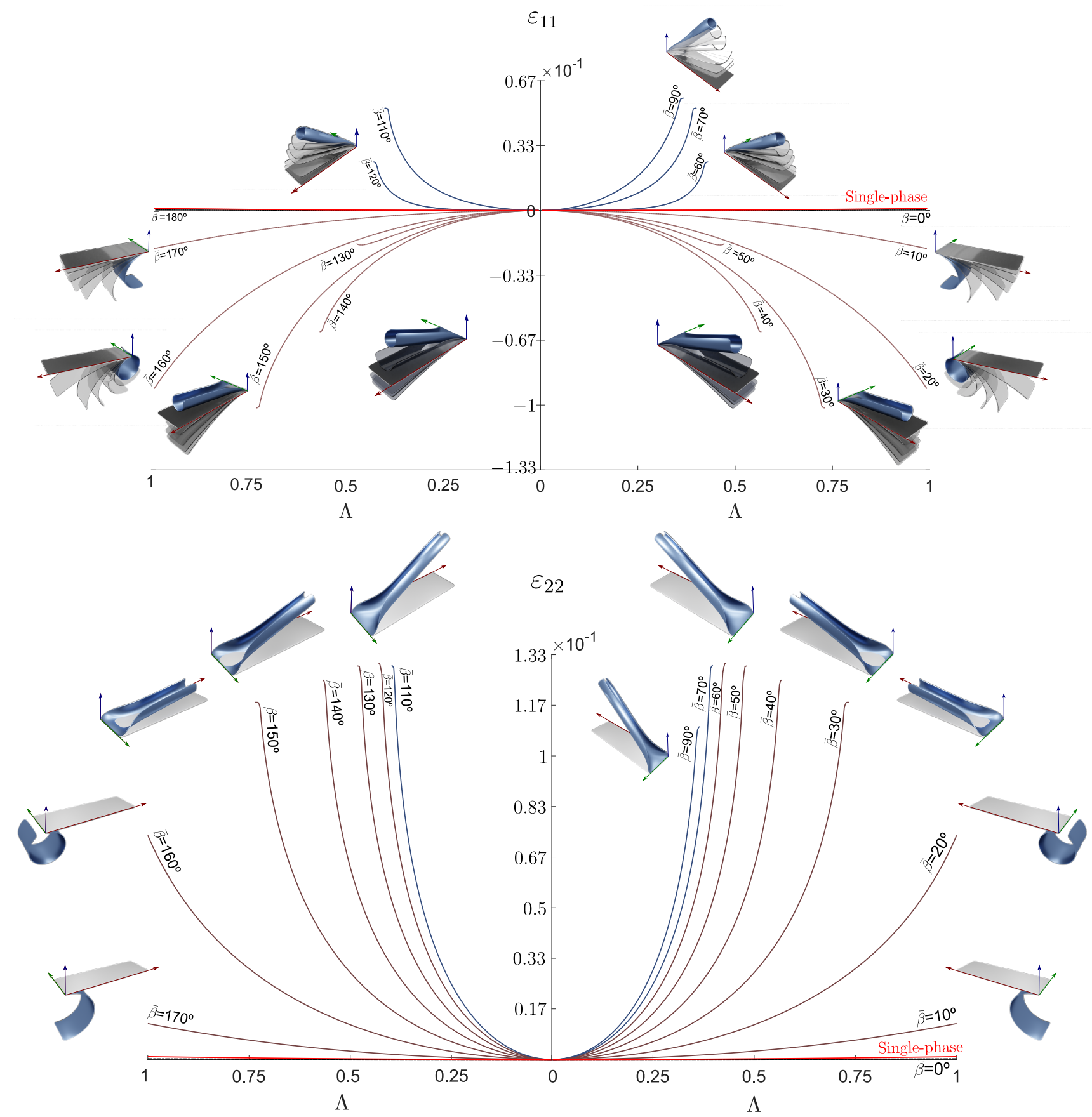

Figure 14: Numerical example 3. Components $\varepsilon_{11}$ and $\varepsilon_{22}$ of the normalised integral value of the Green-Lagrange strain tensor $\varepsilon$ (113), with variation of angle $\bar{\beta}$, keeping $\bar{\alpha}=0^{\circ}$ fixed.

which is plotted in Figure 16. It is observed that no areas of macroscale loss of ellipticity arise, indicating that no spurious mesh dependency effects arise. Similar conclusions were observed for the rest of the simulations presented in this section and thus are not included. In addition, a metric is defined in order to measure the potential loss of convexity of the homogenised model, defined as the smallest eigenvalue of the Hessian operator of the homogenised internal energy, namely

$$
\mathrm{I}_{\mathrm{conv}}=\frac{1}{\bar{\mu}} \min \operatorname{eig}\left(\left[\mathbb{H}_{e}\right]\right),
$$

with $\bar{\mu}$ given by (106)a. This quantity is represented in Figure 17. In this case, some negative values are observed, indicating that local loss of convexity is potentially observed, yet with no 

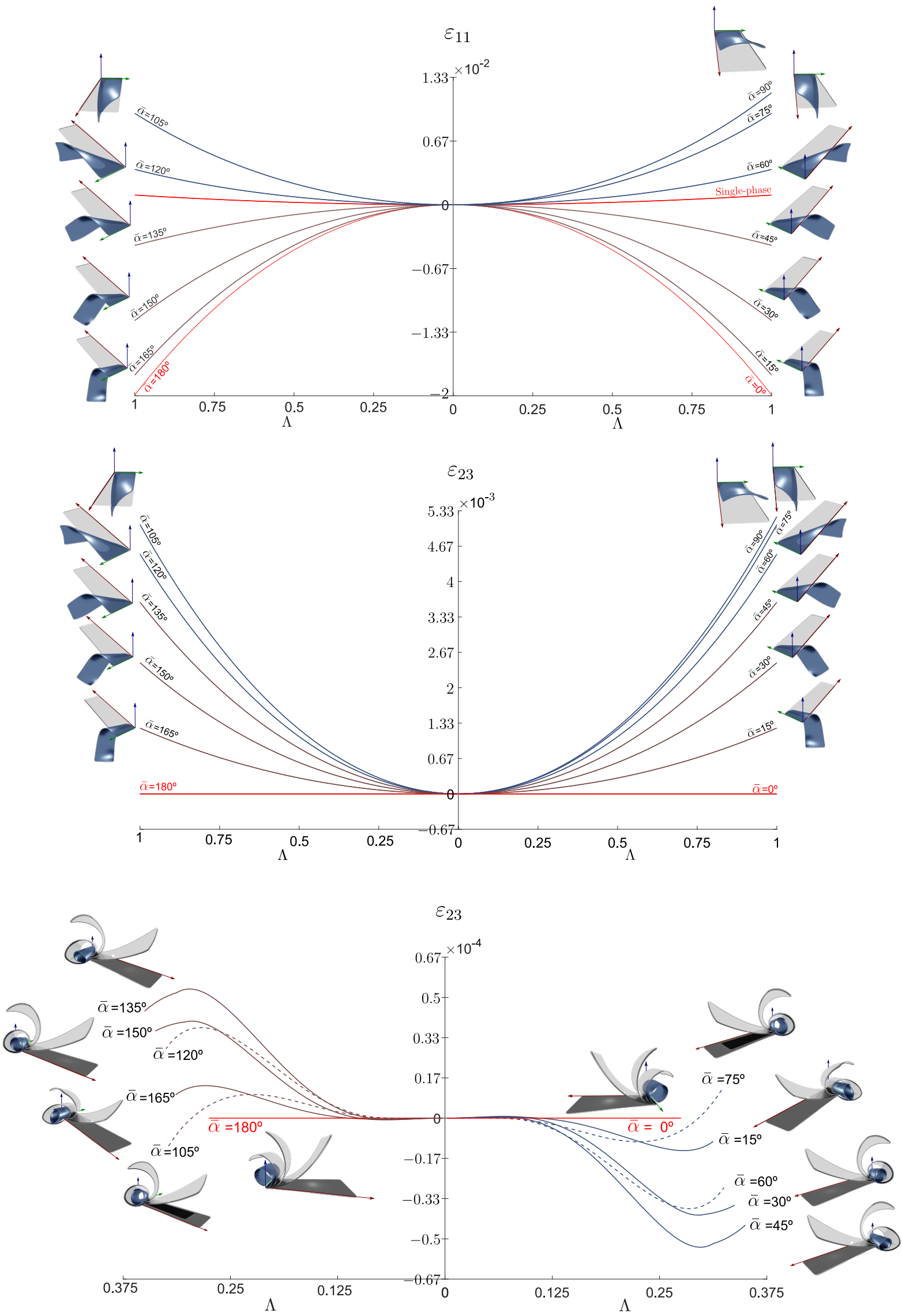

Figure 15: Numerical example 3. Components $\varepsilon_{11}$ and $\varepsilon_{23}$ of the normalised integral value of the Green-Lagrange strain tensor $\varepsilon(113)$, for values of angle $\bar{\alpha}$. The top and middle pictures correspond to $\bar{\beta}=10^{\circ}$, while the bottom one is the case $\bar{\beta}=90^{\circ}$. 
impact in the loss of ellipticity.

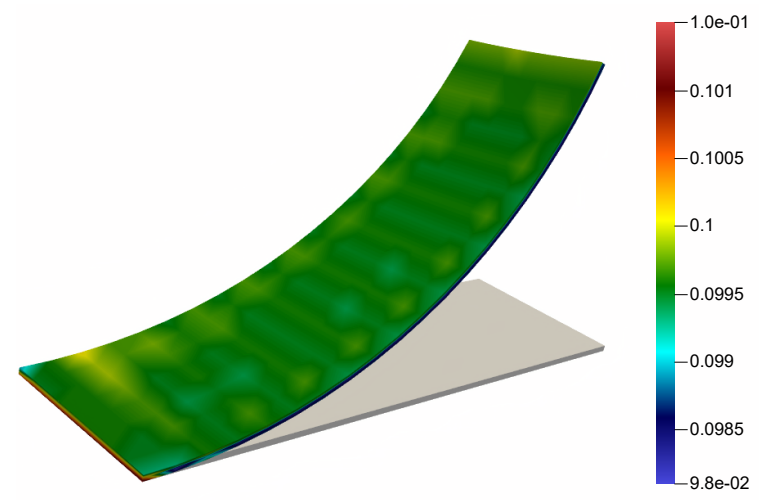

(a)

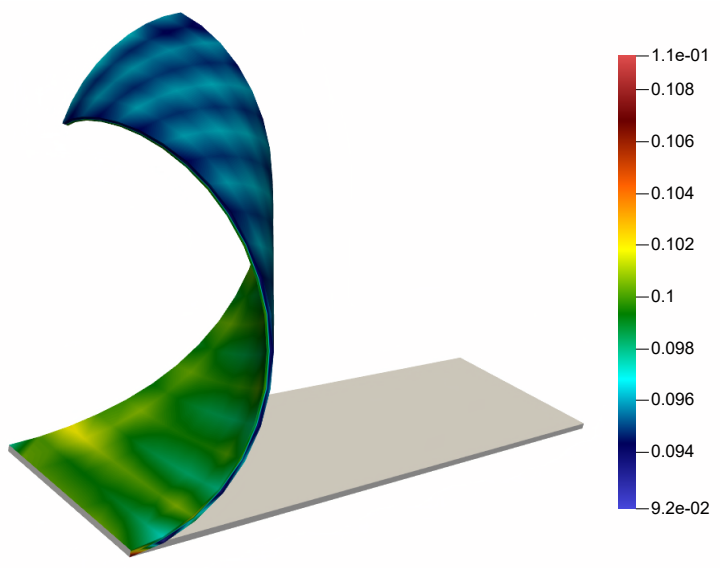

(b)

Figure 16: Numerical example 3. Contour plot of $\mathrm{I}_{\text {ellip }}$ (114) for two load factors: (a) $\Lambda=0.1$, (b) $\Lambda=0.2$. The orientation of the laminate is given by $\bar{\alpha}=15^{\circ}$ and $\bar{\beta}=90^{\circ}$.

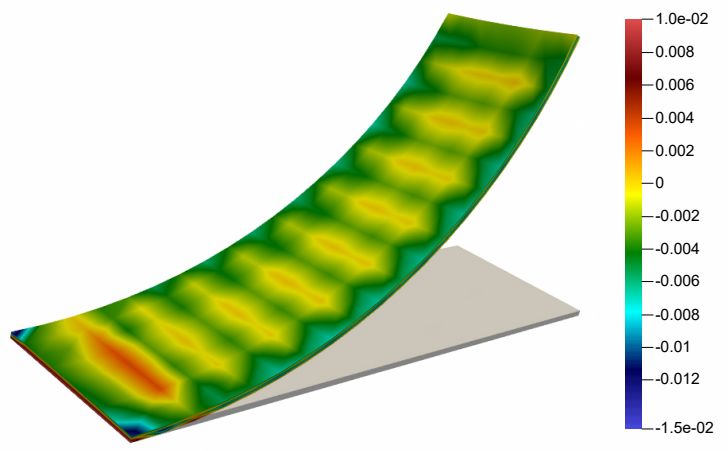

(a)

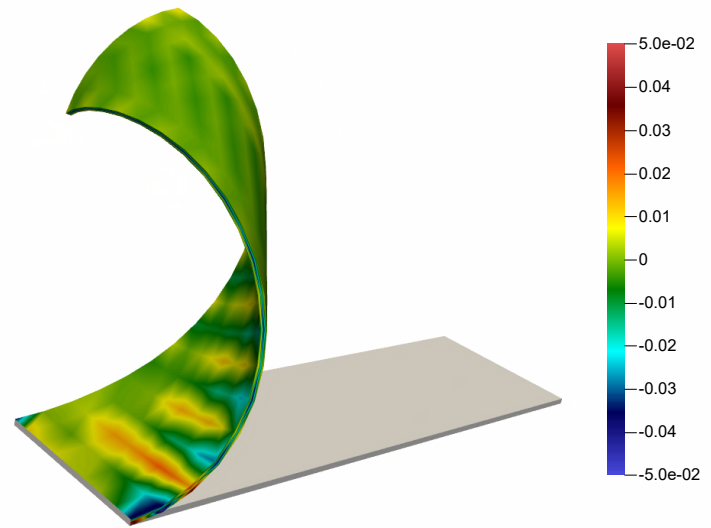

(b)

Figure 17: Numerical example 3. Contour plot of $\mathrm{I}_{\text {conv }}$ (115) for two load factors: (a) $\Lambda=0.1$, (b) $\Lambda=0.2$. The orientation of the laminate is given by $\bar{\alpha}=15^{\circ}$ and $\bar{\beta}=90^{\circ}$.

\subsection{Numerical example 4: buckling on a multi-layered DE square membrane}

The objectives of this example are to:

- Detect the onset of instabilities on a rank-one DE laminated composite square membrane.

- Observe the different patterns of buckling in terms of the microscale configuration.

- Analyse possible local loss of ellipticity and convexity.

The geometry for this numerical example is given by a square membrane of side $l=0.06 \mathrm{~m}$ and thickness $h=0.001 \mathrm{~m}$, clamped along all its side faces, as represented in Figure 18. The membrane is subjected to a prescribed electric surface charge on its base, while it is grounded to zero potential on its topside. Geometrical and Finite Element simulation parameters are presented in Table 6. Q3 Finite Elements are used to interpolate both displacement and electric potential, being 104, 188 the total number of degrees of freedom, and the surface charge is applied incrementally, being $\Lambda \in[0,1]$ the load factor. 


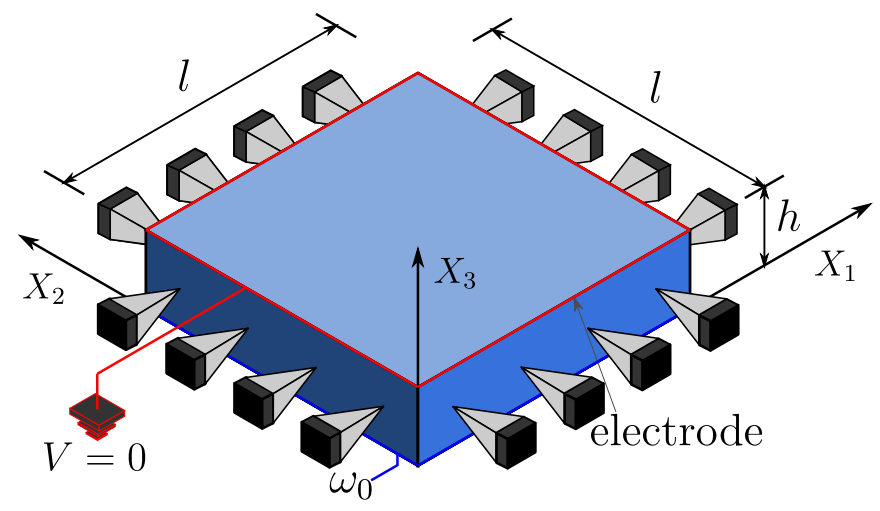

Figure 18: Numerical example 4. Geometry and boundary conditions.

\begin{tabular}{lccclcc}
\hline Geometrical parameters & $l$ & 0.06 & $\mathrm{~m}$ & Simulation parameters & $N_{x}$ & 60 \\
& $h$ & 0.001 & $\mathrm{~m}$ & & $N_{y}$ & 60 \\
& & & & & $N_{z}$ & 2 \\
Electric charge & $\omega_{0}$ & 0.02 & $\mathrm{C} / \mathrm{m}^{2}$ & Newton tolerance & & $10^{-6}$ \\
\hline
\end{tabular}

Table 6: Numerical example 4. Geometrical and simulation parameters.

The constituents of the laminated composite material follow a Mooney-Rivlin model for the mechanical contribution, and an ideal dielectric for the electromechanical contribution. The parameters of the model for each phase are included in Table 7 . The contrast between mechanical properties is $f_{m}=4.06$ and that between electrical properties is $f_{e}=4$.7. Note that the materials of the phases correspond to VHB-4910 and Elastosil RT-625 [26] for the soft matrix and the stiff reinforcement, respectively.

\begin{tabular}{llclcl}
\hline Properties & \multicolumn{2}{l}{ Phase $a$} & \multicolumn{2}{l}{ Phase $b$} & Units \\
\hline Mechanical parameters & $\mu_{1}^{a}$ & $1.0 \times 10^{5}$ & $\mu_{1}^{b}$ & $4.1 \times 10^{5}$ & $\mathrm{~Pa}$ \\
& $\mu_{2}^{a}$ & 0 & $\mu_{2}^{b}$ & 0 & $\mathrm{~Pa}$ \\
& $\lambda^{a}$ & $3.0 \times 10^{5}$ & $\lambda^{b}$ & $1.2 \times 10^{6}$ & $\mathrm{~Pa}$ \\
Electrical parameters & $\epsilon_{r}^{a}$ & 4.8 & $\epsilon_{r}^{b}$ & 22.6 & - \\
Concentration & $c^{a}$ & 0.5 & $c^{b}$ & 0.5 & - \\
\hline
\end{tabular}

Table 7: Numerical example 4. Material parameters of the model for each phase (see $(27),(18)$ ).

A similar study was conducted in [32], where a circular membrane, made of a single-phase DE, was subjected to electrical stimuli in order to observe the development of instabilities as it deforms. In this work, we explore a different square geometry and study the influence of introducing a more complex rank-one laminate arrangement. As the square membrane is subjected to an increasing electric potential, it starts to slowly bend upwards until it reaches a point where it develops some initial buckling, marking a drastic change in stiffness. In Figure 19, the equilibrium paths are displayed for different orientations of the laminate, keeping the angle $\bar{\alpha}=0^{\circ}$ fixed and varying the angle $\bar{\beta}$ from $0^{\circ}$ to $90^{\circ}$. The vertical displacement along the $X_{3}$ axis $\left(u_{3}\right)$ is plotted for two sample points on the membrane: test point 1 , which corresponds to the centre of the square, and test point 2, which is close to the boundary.

In Figure 19(a), the onset of a first order buckling is captured at approximately $\Lambda=0.01$, being the behaviour of deformation quite similar for all laminate orientations of the composite. In Figure 19(b), a second-order buckling starting around $\Lambda=0.15$ is observed. In this case, it is 
demonstrated that the orientation of the laminate does affect significantly the buckling pattern, being the deformation for angles $0^{\circ}<\bar{\beta}<45^{\circ}$ larger than for higher values of the angle $\bar{\beta}$.

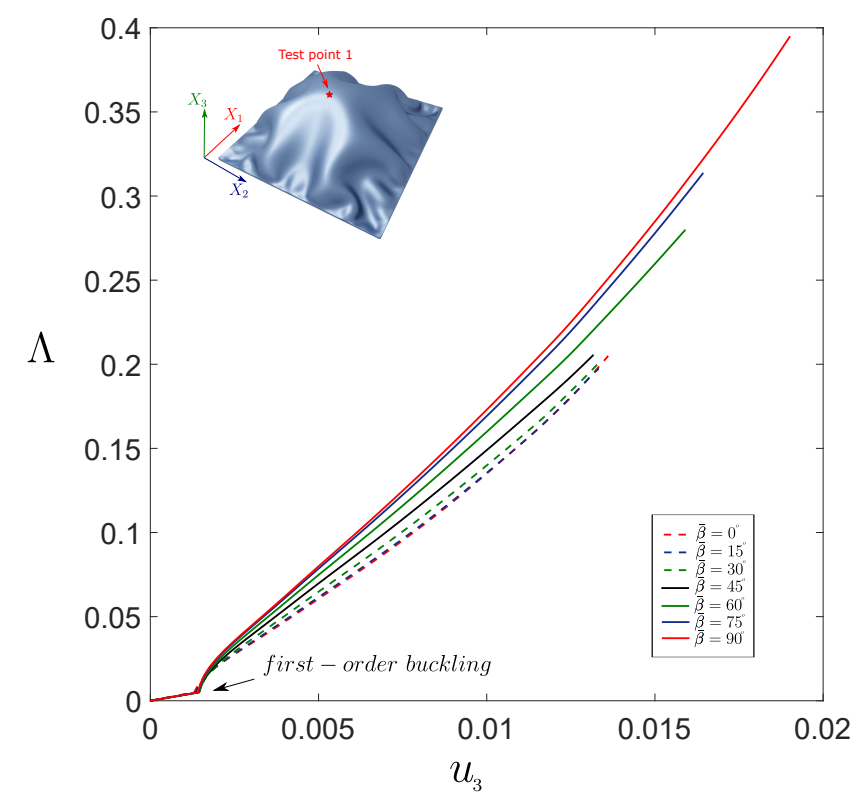

(a)

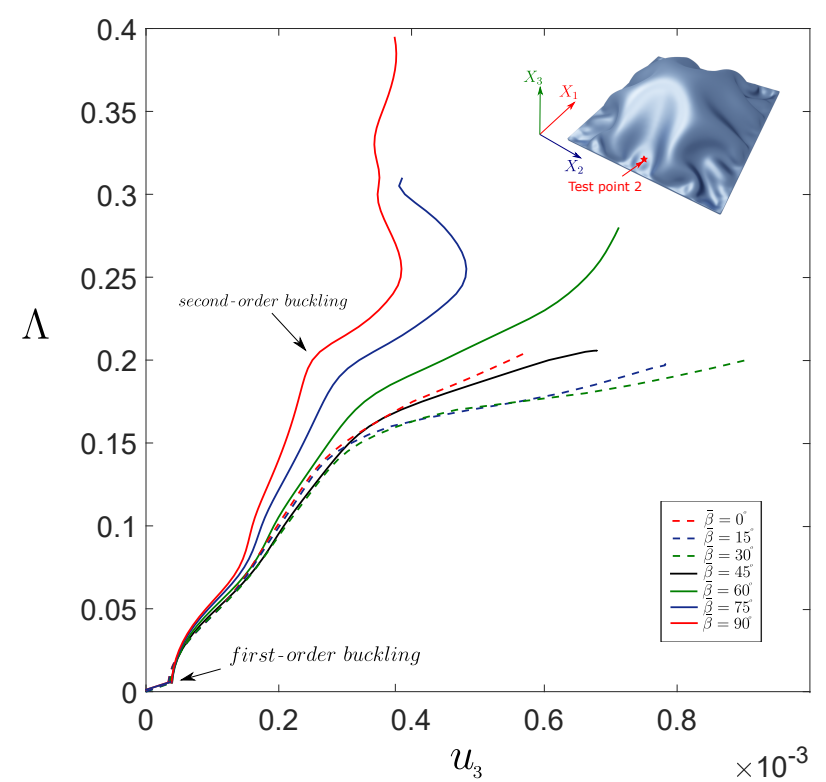

(b)

Figure 19: Numerical example 4. Vertical displacement along the $X_{3}$ axis of two test points: (a) the centre of the square; (b) a point near the border.

In Figure 20, some snapshots of the deformation evolution of the membrane are represented, for the composite with laminate orientation given by $\bar{\alpha}=0^{\circ}, \bar{\beta}=75^{\circ}$. It is observed how the membrane buckles initially around its centre, and then the density of small instabilities quickly increases with the loading factor.

In Figure 21, the final aspect of the square membrane after deformation is shown for different laminate orientations of the composite. The first two rows analyse the effect of the change in orientation angle $\bar{\beta}$ whilst keeping $\bar{\alpha}=0^{\circ}$, whereas the last two rows focus on the variation of $\bar{\alpha}$ whilst keeping $\bar{\beta}=75^{\circ}$. On the one hand, from the study of the different $\bar{\beta}$ cases, it can be inferred that the larger the angle $\bar{\beta}$ is, the more complex the deformation pattern seems to be, showing a greater amount of wrinkles within the membrane. On the other hand, the membrane seems to have a smoother deformation for intermediate values of $\bar{\alpha}$, whereas values of $\bar{\alpha}$ near the extremes of the interval $\left[0^{\circ}, 90^{\circ}\right]$ cause a larger amount of instabilities.

Next, in Figures 22 and 23, the norm of the microstructure amplitude vectors $\|\boldsymbol{\alpha}\|$ and $\|\boldsymbol{\beta}\|$ is represented for various laminate orientation angles $\bar{\beta}$, whilst keeping $\bar{\alpha}=0^{\circ}$ fixed. Specifically, $\|\boldsymbol{\alpha}\|$ is represented in Figure 22, where it is observed how its magnitude globally increases with the orientation angle $\bar{\beta}$, specially in the areas near the instabilities. In Figure $23,\|\boldsymbol{\beta}\|$ is plotted, observing that it also increases with the angle $\bar{\beta}$.

Finally, a study of the possible loss of ellipticity and convexity is conducted. Figure 24 illustrates the spatial distribution of the smallest of the minors of the acoustic tensor for any unit normal orientation, namely $\mathrm{I}_{\text {ellip }}(114)$, for two deformation states, $(\Lambda=0.25)$ and $(\Lambda=0.3)$. It is ascertained that no areas of loss of ellipticity are observed, indicating that no anomalous mesh dependency effects can take place. Moreover, Figure 25 shows a contour plot of the minimum eigenvalue of the homogenised Hessian operator of the internal energy density, $\mathrm{I}_{\text {conv }}$ (115), for the same two deformation states. In this case, some negative values are observed, indicating that loss of convexity is potentially developed, yet with no impact in the loss of ellipticity. 


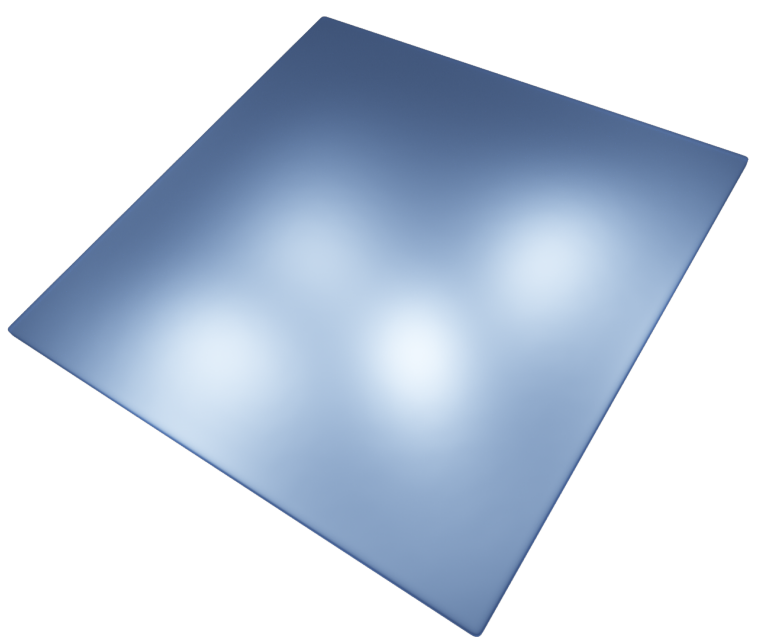

(a)

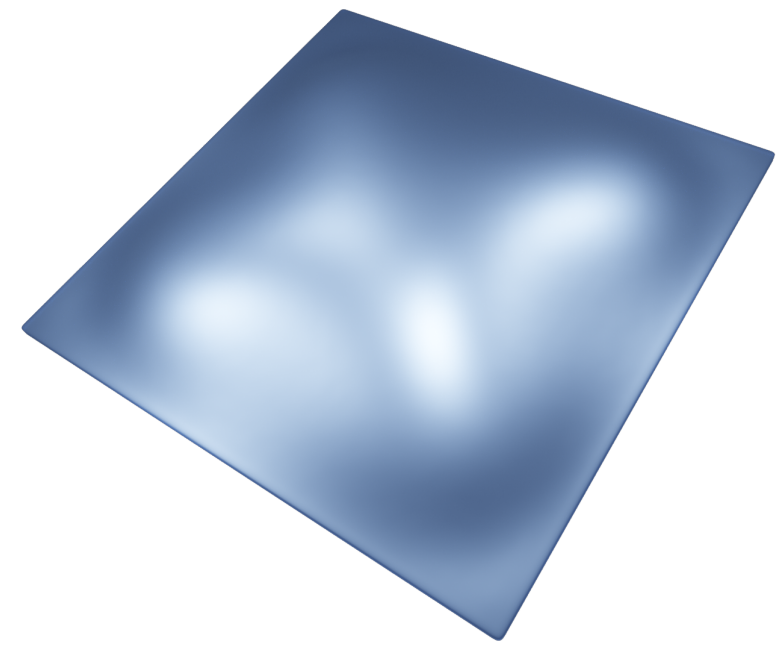

(c)

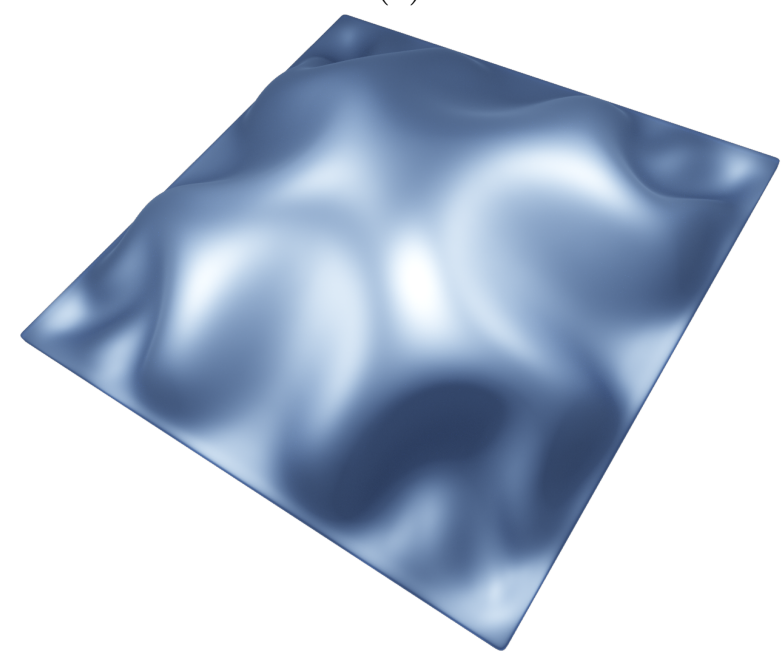

(e)

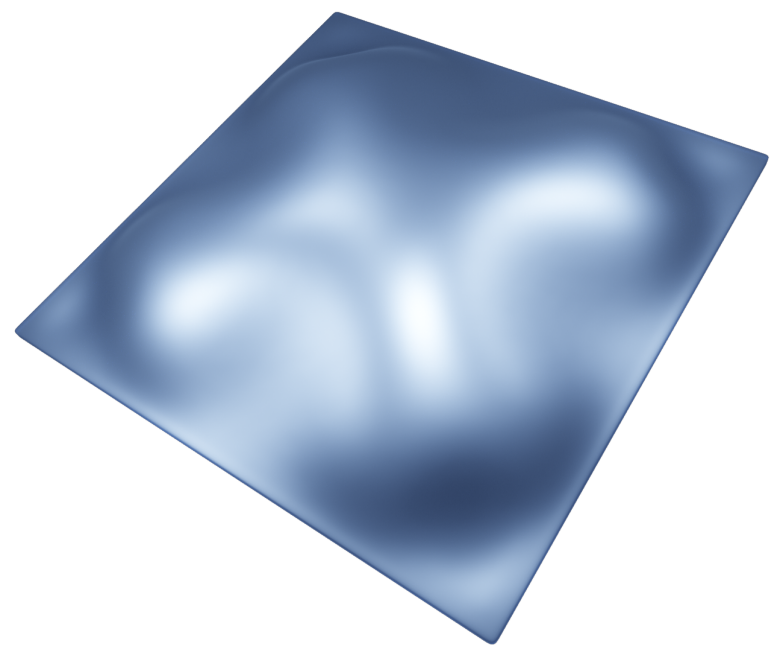

(b)

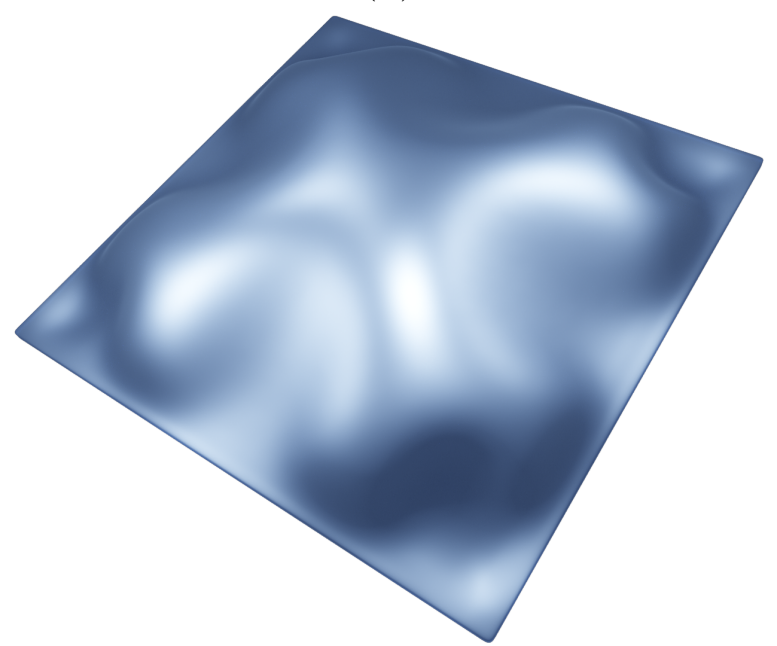

(d)

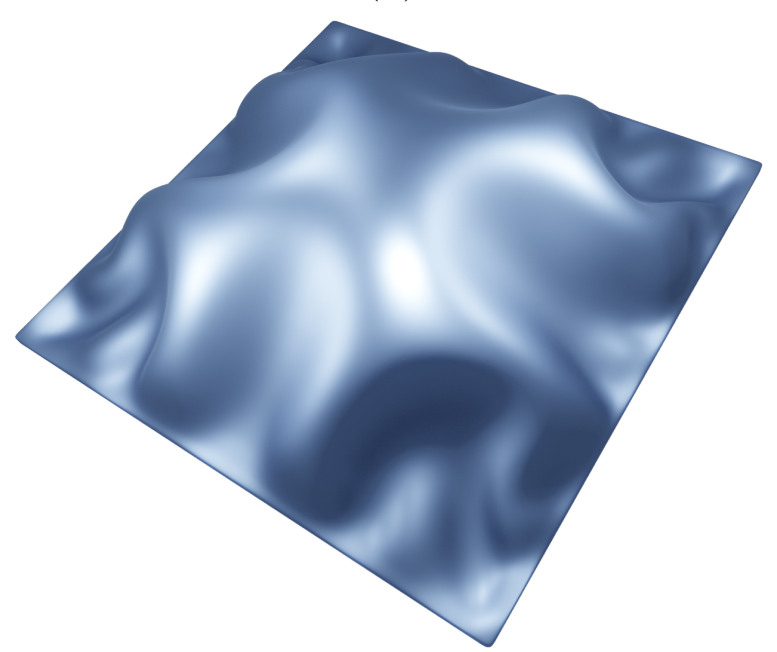

(f)

Figure 20: Numerical example 4. Evolution of deformation with the load factor for the case $\bar{\alpha}=0^{\circ}, \bar{\beta}=75^{\circ}$. From left to right and from top to bottom, the snapshots correspond to the following load factors: (a) $\Lambda=0.05$, (b) $\Lambda=0.1$, (c) $\Lambda=0.15$, (d) $\Lambda=0.2$, (e) $\Lambda=0.25$, (f) $\Lambda=0.3$.

\section{Conclusions}

In this paper, a new Finite Element based computational framework for the numerical simulation of rank-one multi-layered electro-active polymers undergoing large deformations and electric 

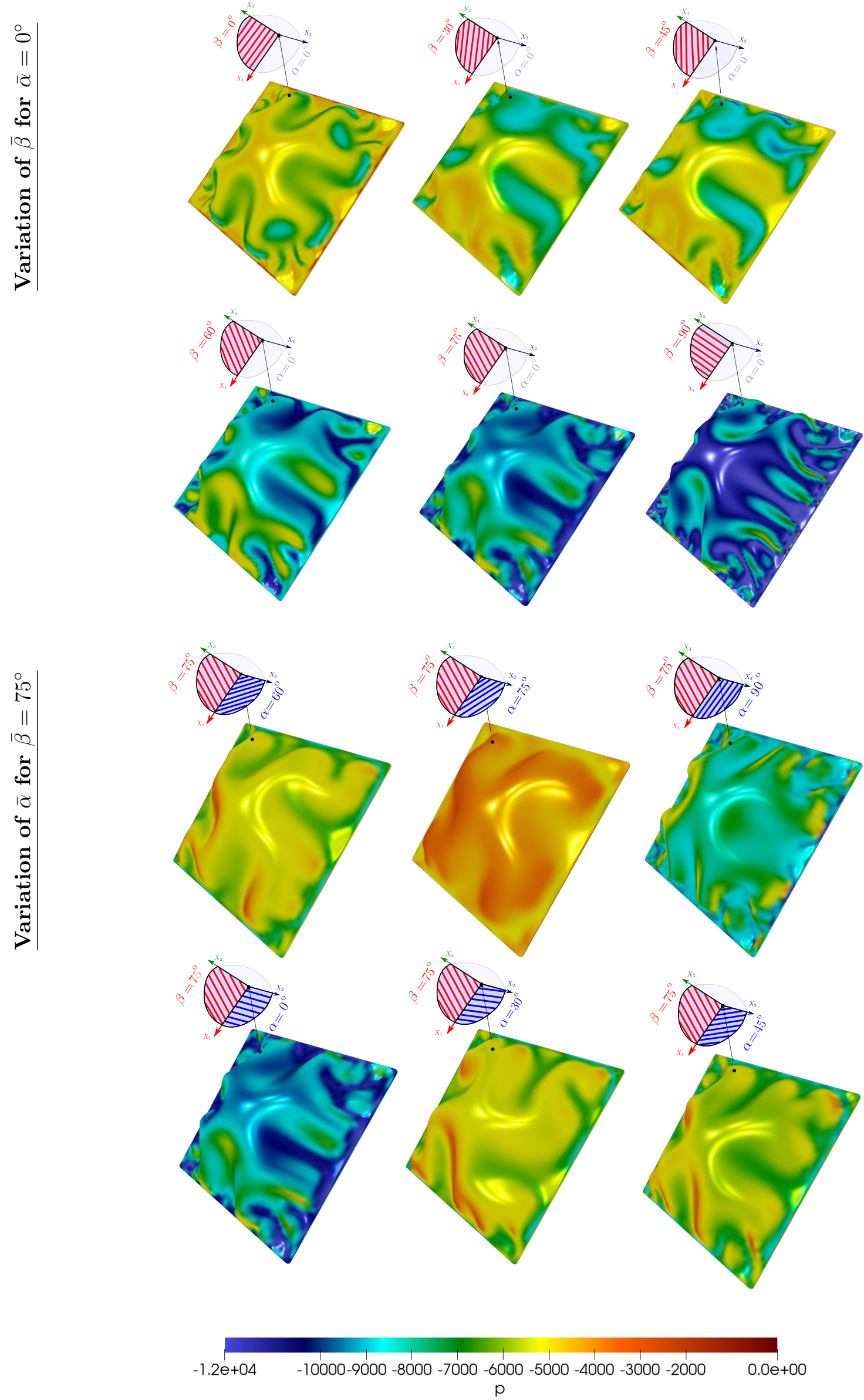

Figure 21: Numerical example 4. Different patterns of buckling for (a) various values of $\bar{\beta}=$ $\left[0^{\circ}, 30^{\circ}, 45^{\circ}, 60^{\circ}, 75^{\circ}, 90^{\circ}\right]$ and $\bar{\alpha}=0^{\circ}$, and (b) various values of $\bar{\alpha}=\left[0^{\circ}, 30^{\circ}, 45^{\circ}, 60^{\circ}, 75^{\circ}, 90^{\circ}\right]$ and $\bar{\beta}=0^{\circ}$. 

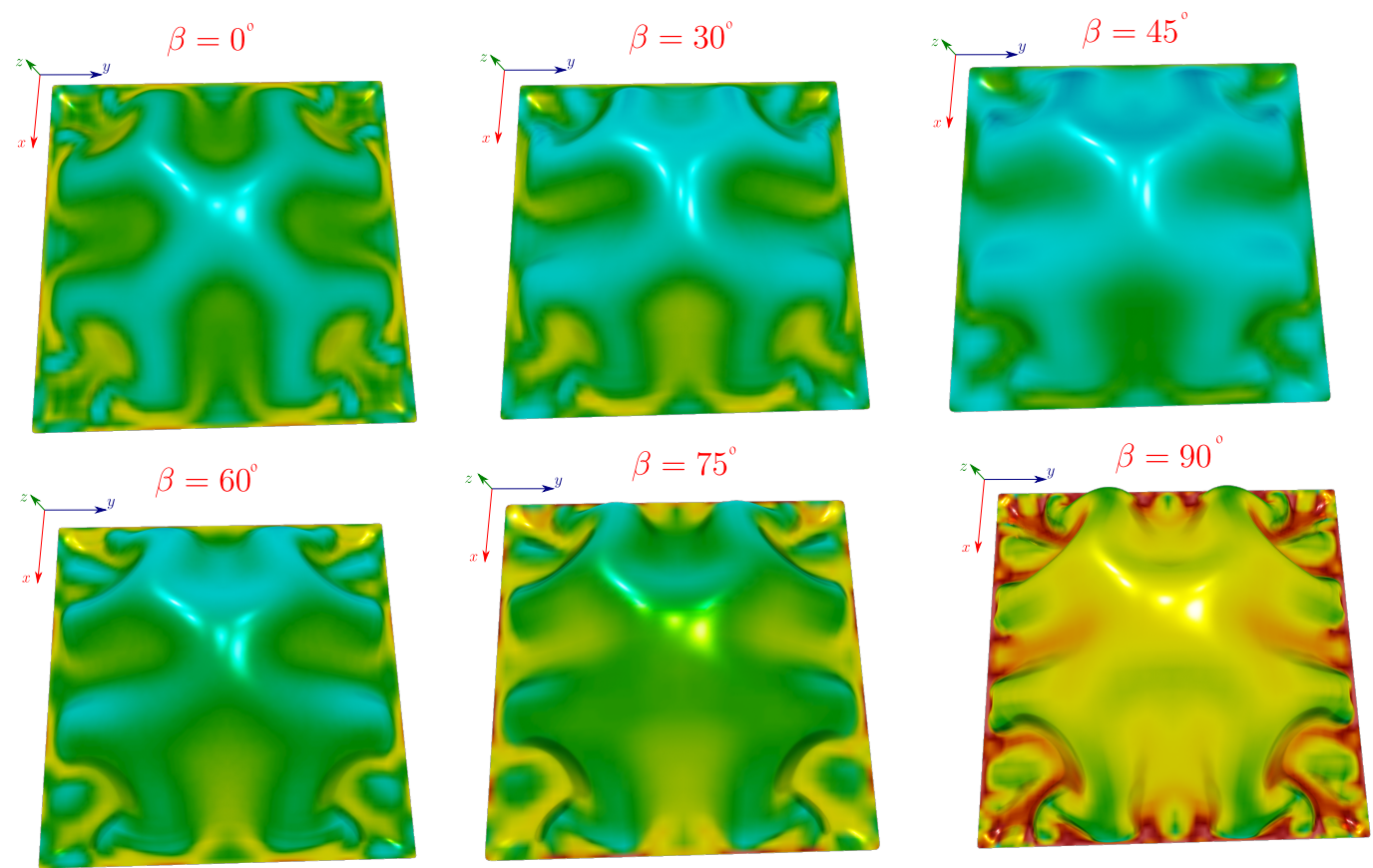

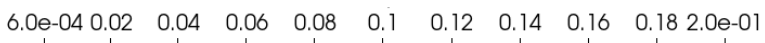

Figure 22: Magnitude of the microstructure $\boldsymbol{\alpha}$ vector, for different values of $\bar{\beta}$ angle, keeping $\alpha=0^{\circ}$ fixed.
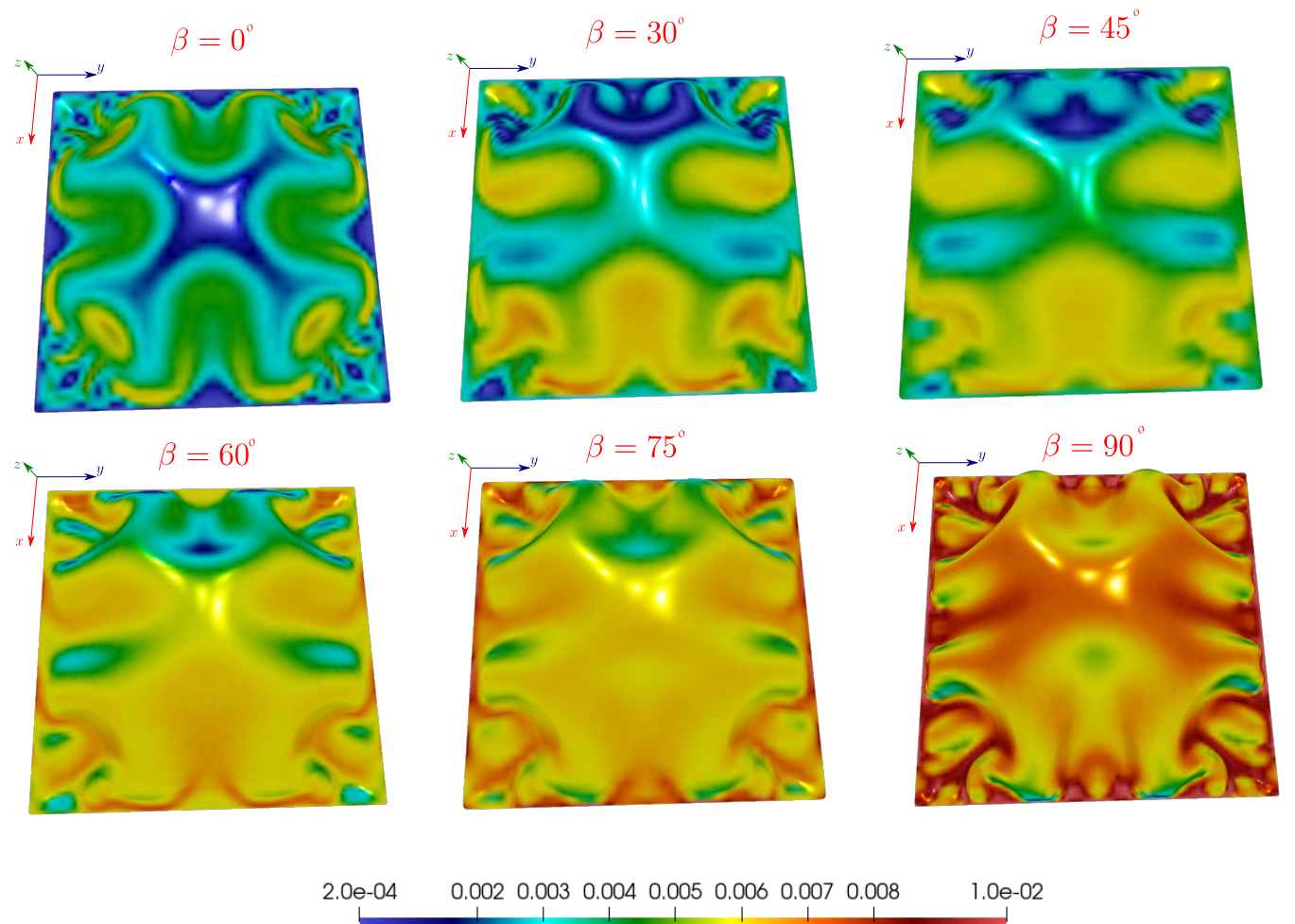

Figure 23: Magnitude of the microstructure $\boldsymbol{\beta}$ vector, for different values of $\bar{\beta}$ angle, keeping $\bar{\alpha}=0^{\circ}$ fixed.

fields has been introduced. Following the well-established homogenisation strategy for rank-n laminates [17, 18], the paper exploits the use of Convex Multi-Variable (CMV) energy density functionals [31] for each of the individual material phase components in order to ensure the existence of solutions of the microstructure problem, defined in terms of the so-called deformation gradient and electric displacement amplitude vectors $\{\boldsymbol{\alpha}, \boldsymbol{\beta}\}$. 


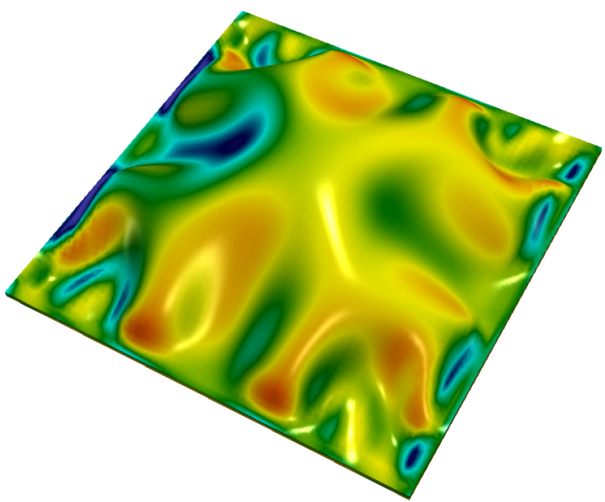

(a)

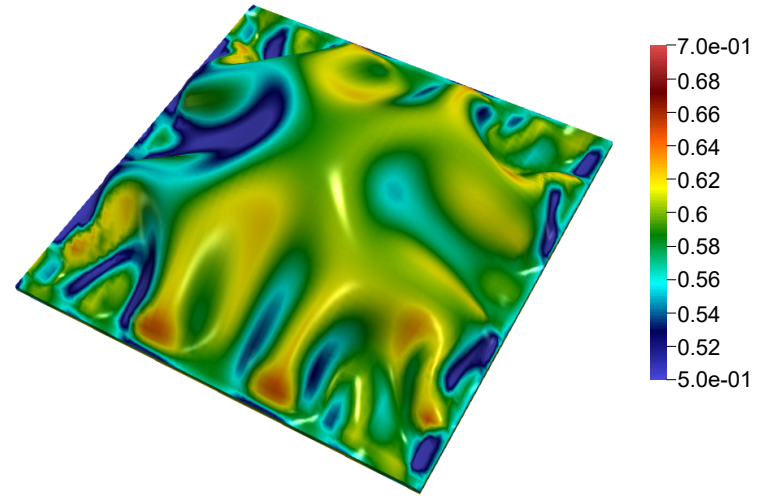

(b)

Figure 24: Numerical example 4. Contour plot of $\mathrm{I}_{\text {ellip }}$ (114) for (a) $\Lambda=0.25$ and (b) $\Lambda=0.3$. The orientation of the laminate is given by $\bar{\alpha}=0^{\circ}$ and $\bar{\beta}=75^{\circ}$.

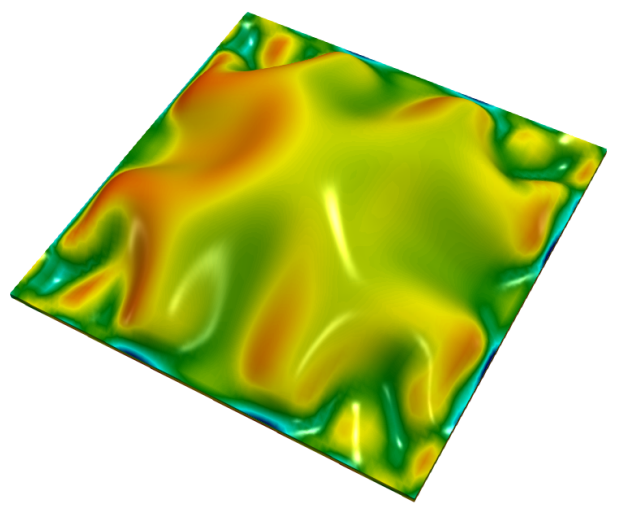

(a)
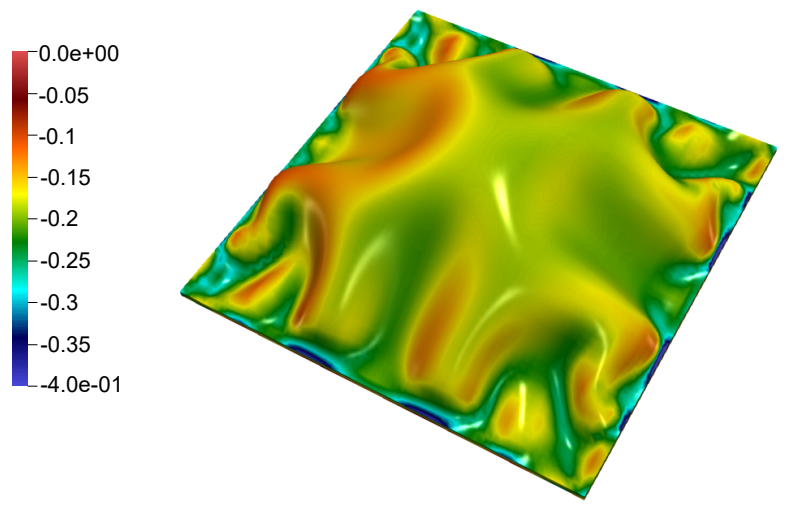

(b)

Figure 25: Numerical example 4. Contour plot of $\mathrm{I}_{\text {conv }}$ (115) for (a) $\Lambda=0.25$ and (b) $\Lambda=0.3$. The orientation of the laminate is given by $\bar{\alpha}=0^{\circ}$ and $\bar{\beta}=75^{\circ}$.

Undesired mesh dependency, introduced by the possible loss of ellipticity of the homogenised constitutive model, is strictly monitored via the evolution of the minors of the acoustic tensor throughout the entire deformation process. The high nonlinearity of the coupled electromechanical problem is resolved through a monolithic multi-scale (micro and macro) NewtonRaphson algorithm enhanced via a tailor-made arc-length technique, critical in order to bypass the geometrical instabilities and thus harness the potential of these materials in the very large strain regime. Very importantly, the complexity of the algebra is dramatically simplified via the use of both a tensor cross product operation between vectors and tensors and an unconventional additive decomposition of the homogenised deformation gradient.

The computational strategy presented enables the exploration of very complex deformation patterns (e.g., combined bending/torsion/stretching), way beyond the onset of geometrical instabilities and without the need to assume any simplifications in the kinematics, such as plane strain or exact incompressibility. The paper includes a series of challenging numerical examples seeking to explore the performance of these composite materials at both micro and macro scales. The effect that the micro-structure composition (i.e., orientation and contrast) can potentially have in the response of the composite at different levels of deformation (or electric field) is studied. In this sense, the manuscript paves the way for the prospective study of realistic miniaturised soft robotics through the consideration of rank-n multi-layered electro-active polymers. With this in mind, our future lines of work will include the extension of this computational framework to lam- 
inated composites of higher order as well as the consideration of possible thermal and viscoelastic effects upon the response of the material.

\section{Acknowledgements}

Project supported by the Autonomous Community of the Region of Murcia through the program for the development of scientific and technical research by competitive groups (20911/PI/18), included in the Regional Program for the Promotion of Scientific and Technical Research of Fundación Séneca - Agencia de Ciencia y Tecnología de la Región de Murcia. The first author also acknowledges the support provided by Fundación Séneca - Agencia de Ciencia y Tecnología de la Región de Murcia, for the award of a Postdoc Fellowship with reference 20818/PD/18. The third author also acknowledges the support provided by Ministerio de Ciencia, Innovación y Universidades, for the award of a Juan de la Cierva Formación Fellowship, and by Fundación Séneca - Agencia de Ciencia y Tecnología de la Región de Murcia, through the contract with reference number 21132/SF/19 under the program "Subprograma Regional Saavedra Fajardo de Incorporacion de Doctores a Universidades y Centros de Investigacion de la Region De Murcia". The fourth author acknowledges the financial support received through the European Training Network Protechtion (Project ID: 764636).

\section{References}

[1] J. M. Ball. Convexity conditions and existence theorems in nonlinear elasticity. Arch Ration Mech Anal, 63(4):337-403, 1976.

[2] J. M. Ball. Some open problems in elasticity. In Geometry, Mechanics, and Dynamics, pages 3-59. Springer-Verlag, 2002.

[3] Y. Bar-Cohen. EAP history, current status, and infrastructure in Y. Bar-Cohen, Electroactive Polymer (EAP) Actuators as Artificial Muscles, chapter 1, pages 3-44. SPIE press, 2001.

[4] Y. Bar-Cohen. Electroactive polymers (EAP) as actuators for potential future planetary mechanisms. In Proceedings. NASA/DoD Conference on Evolvable Hardware, 2004. IEEE, 2004.

[5] K. Bertoldi and M. Gei. Instabilities in multilayered soft dielectrics. J Mech Phys Solids, 59 (1):18-42, 2011.

[6] D. Bishara and M. Jabareen. A reduced mixed finite-element formulation for modeling the viscoelastic response of electro-active polymers at finite deformation. Mathematics and Mechanics of Solids, 24(5):1578-1610, 2018.

[7] J. Bonet, A. J. Gil, and R. Ortigosa. A computational framework for polyconvex large strain elasticity. Comput Methods Appl Mech Eng, 283:1061-1094, 2015.

[8] J. Bonet, A. J. Gil, and R. Ortigosa. On a tensor cross product based formulation of large strain solid mechanics. International Journal of Solids and Structures, 84:49-63, 2016.

[9] J. Bonet, A. J. Gil, and R. D. Wood. Nonlinear Solid Mechanics for Finite Element Analysis: Statics. Cambridge University Press, 2016. ISBN 1107115795.

[10] E. Bortot, R. Denzer, A. Menzel, and M. Gei. Analysis of viscoelastic soft dielectric elastomer generators operating in an electrical circuit. Int J Solids Struct, 78-79:205-215, 2016. 
[11] R. Bustamante. Transversely isotropic non-linear electro-active elastomers. Acta Mech, 206 (3-4):237-259, 2008.

[12] F. Carpi and E. Smela, editors. Biomedical Applications of Electroactive Polymer Actuators. John Wiley \& Sons, Ltd, 2009.

[13] F. Carpi, D. De Rossi, R. Kornbluh, R. Pelrine, and P. Sommer-Larsen. Dielectric Elastomers as Electromechanical Transducers. Elsevier, 2008.

[14] F. Carpi, G. Frediani, S. Turco, and D. De Rossi. Bioinspired tunable lens with muscle-like electroactive elastomers. Adv Funct Mater, 21(21):4152-4158, 2011.

[15] R. de Boer. Vektor-und Tensorrechnung für Ingenieure. Springer-Verlag, 1982.

[16] E. A. de Souza Neto, D. Peri, and D. R. J. Owen. Computational Methods for Plasticity. John Wiley \& Sons, Ltd, 2008.

[17] G. deBotton and I. Hariton. High-rank nonlinear sequentially laminated composites and their possible tendency towards isotropic behavior. J Mech Phys Solids, 50(12):2577-2595, 2002 .

[18] G. deBotton, L. Tevet-Deree, and E. A. Socolsky. Electroactive heterogeneous polymers: Analysis and applications to laminated composites. Mech Adv Mater Struct, 14(1):13-22, 2007.

[19] G. deBotton. Transversely isotropic sequentially laminated composites in finite elasticity. $J$ Mech Phys Solids, 53(6):1334-1361, 2005.

[20] G. deBotton and L. Tevet-Deree. Electroactive polymer composites: analysis and simulation. In W. D. Armstrong, editor, Smart Structures and Materials 2006: Active Materials: Behavior and Mechanics. SPIE, 2006.

[21] A. Dorfmann and R. W. Ogden. Nonlinear electroelasticity. Acta Mech, 174(3-4):167-183, 2005.

[22] A. Dorfmann and R. W. Ogden. Nonlinear electroelastic deformations. J Elast, 82(2):99-127, 2006.

[23] M. Franke, R. Ortigosa, A. Janz, A. Gil, and P. Betsch. A mixed variational framework for the design of energy-momentum integration schemes based on convex multi-variable electroelastodynamics. Comput Methods Appl Mech Eng, 351:109-152, 2019.

[24] J. Furer and P. Ponte Castañeda. Macroscopic instabilities and domain formation in neohookean laminates. Journal of the Mechanics and Physics of Solids, 118:98-114, 2018.

[25] P. I. Galich and S. Rudykh. Manipulating pressure and shear waves in dielectric elastomers via external electric stimuli. Int J Solids Struct, 91:18-25, 2016.

[26] P. I. Galich and S. Rudykh. Shear wave propagation and band gaps in finitely deformed dielectric elastomer laminates: Long wave estimates and exact solution. J Appl Mech, 84(9), 2017.

[27] P. I. Galich, V. Slesarenko, and S. Rudykh. Shear wave propagation in finitely deformed 3d fiber-reinforced composites. Int J Solids Struct, 110-111:294-304, 2017. 
[28] M. Gei and K. C. Mutasa. Optimisation of hierarchical dielectric elastomer laminated composites. Int J Non Linear Mech, 106:266-273, 2018.

[29] M. Gei, R. Springhetti, and E. Bortot. Performance of soft dielectric laminated composites. Smart Mater Struct, 22(10):104014, 2013.

[30] G. Geymonat, S. Müller, and N. Triantafyllidis. Homogenization of nonlinearly elastic materials, microscopic bifurcation and macroscopic loss of rank-one convexity. Arch Ration Mech Anal, 122(3):231-290, 1993.

[31] A. J. Gil and R. Ortigosa. A new framework for large strain electromechanics based on convex multi-variable strain energies: Variational formulation and material characterisation. Comput Methods Appl Mech Eng, 302:293-328, 2016.

[32] H. Godaba, Z.-Q. Zhang, U. Gupta, C. C. Foo, and J. Zhu. Instabilities in dielectric elastomers: buckling, wrinkling, and crumpling. Soft Matter, 15(36):7137-7144, 2019.

[33] F. S. Göküzüm, L. T. K. Nguyen, and M.-A. Keip. An artificial neural network based solution scheme for periodic computational homogenization of electrostatic problems. Mathematical and Computational Applications, 24(2):40, 2019.

[34] F. S. Göküzüm, L. T. K. Nguyen, and M.-A. Keip. A multiscale FE-FFT framework for electro-active materials at finite strains. Computational Mechanics, 64(1):63-84, 2019.

[35] O. Gonzalez and A. M. Stuart. A First Course in Continuum Mechanics. Cambridge University Press, 2001.

[36] A. Goshkoderia and S. Rudykh. Electromechanical macroscopic instabilities in soft dielectric elastomer composites with periodic microstructures. Eur J Mech A Solids, 65:243-256, 2017.

[37] C. Huang and Q. Zhang. Enhanced dielectric and electromechanical responses in high dielectric constant all-polymer percolative composites. Adv Funct Mater, 14(5):501-506, 2004.

[38] C. Huang, Q. M. Zhang, G. deBotton, and K. Bhattacharya. All-organic dielectric-percolative three-component composite materials with high electromechanical response. Appl Phys Lett, 84(22):4391-4393, 2004.

[39] M. Itskov and V. N. Khiêm. A polyconvex anisotropic free energy function for electro- and magneto-rheological elastomers. Mathematics and Mechanics of Solids, 21(9):1126-1137, 2016.

[40] M. Jabareen. On the modeling of electromechanical coupling in electroactive polymers using the mixed finite element formulation. Procedia IUTAM, 12:105-115, 2015.

[41] R. D. Kornbluh, R. Pelrine, Q. Pei, S. Oh, and J. Joseph. Ultrahigh strain response of fieldactuated elastomeric polymers. In Y. Bar-Cohen, editor, Smart Structures and Materials 2000: Electroactive Polymer Actuators and Devices (EAPAD). SPIE, 2000.

[42] R. D. Kornbluh, R. Pelrine, H. Prahlad, A. Wong-Foy, B. McCoy, S. Kim, J. Eckerle, and T. Low. From boots to buoys: promises and challenges of dielectric elastomer energy harvesting. In Y. Bar-Cohen and F. Carpi, editors, Electroactive Polymer Actuators and Devices (EAPAD) 2011. SPIE, 2011.

[43] S. P. Lacour, H. Prahlad, R. Pelrine, and S. Wagner. Mechatronic system of dielectric elastomer actuators addressed by thin film photoconductors on plastic. Sens Actuators, A, 111(2-3):288-292, 2004. 
[44] J. Y. Li, C. Huang, and Q. Zhang. Enhanced electromechanical properties in all-polymer percolative composites. Appl Phys Lett, 84(16):3124-3126, 2004.

[45] J. Li. Exchange coupling in $\mathrm{p}(\mathrm{VDF}-\mathrm{TrFE})$ copolymer based all-organic composites with giant electrostriction. Phys Rev Lett, 90(21), 2003.

[46] T. Li, C. Keplinger, R. Baumgartner, S. Bauer, W. Yang, and Z. Suo. Giant voltage-induced deformation in dielectric elastomers near the verge of snap-through instability. $J$ Mech Phys Solids, 61(2):611-628, 2013.

[47] Z. Liao, M. Hossain, X. Yao, M. Mehnert, and P. Steinmann. On thermo-viscoelastic experimental characterization and numerical modelling of VHB polymer. International Journal of Non-Linear Mechanics, 118:103263, 2020.

[48] T. McKay, B. O’Brien, E. Calius, and I. Anderson. An integrated, self-priming dielectric elastomer generator. Appl Phys Lett, 97(6):062911, 2010.

[49] R. M. McMeeking and C. M. Landis. Electrostatic forces and stored energy for deformable dielectric materials. J Appl Mech, 72(4):581-590, 2004.

[50] C. Miehe, D. Vallicotti, and D. Zäh. Computational structural and material stability analysis in finite electro-elasto-statics of electro-active materials. International Journal for Numerical Methods in Engineering, 102(10):1605-1637, 2015.

[51] A. O'Halloran, F. O'Malley, and P. McHugh. A review on dielectric elastomer actuators, technology, applications, and challenges. J Appl Phys, 104(7):071101, 2008.

[52] R. Ortigosa and A. J. Gil. A new framework for large strain electromechanics based on convex multi-variable strain energies: Conservation laws, hyperbolicity and extension to electro-magneto-mechanics. Comput Methods Appl Mech Eng, 309:202-242, 2016.

[53] R. Ortigosa and A. J. Gil. A new framework for large strain electromechanics based on convex multi-variable strain energies: Finite element discretisation and computational implementation. Comput Methods Appl Mech Eng, 302:329-360, 2016.

[54] R. Ortigosa, A. J. Gil, and C. H. Lee. A computational framework for large strain nearly and truly incompressible electromechanics based on convex multi-variable strain energies. Comput Methods Appl Mech Eng, 310:297-334, 2016.

[55] R. Pelrine. High-speed electrically actuated elastomers with strain greater than 100\%. Science, 287(5454):836-839, 2000.

[56] R. E. Pelrine. Review of artificial muscle approaches. In Third Internationat Symposium on Micro Machine and Human Science, 1992.

[57] R. E. Pelrine, R. D. Kornbluh, and J. P. Joseph. Electrostriction of polymer dielectrics with compliant electrodes as a means of actuation. Sens Actuators, A, 64(1):77-85, 1998.

[58] P. Ponte Castañeda and M. Siboni. A finite-strain constitutive theory for electro-active polymer composites via homogenization. Int J Non Linear Mech, 47(2):293-306, 2012.

[59] R. Poya, A. J. Gil, R. Ortigosa, R. Sevilla, J. Bonet, and W. A. Wall. A curvilinear high order finite element framework for electromechanics: From linearised electro-elasticity to massively deformable dielectric elastomers. Comput Methods Appl Mech Eng, 329:75-117, 2018. 
[60] S. Rudykh, K. Bhattacharya, and G. deBotton. Multiscale instabilities in soft heterogeneous dielectric elastomers. Proceedings of the Royal Society A: Mathematical, Physical and Engineering Sciences, 470(2162):20130618, 2014.

[61] S. Rudykh and G. deBotton. Stability of anisotropic electroactive polymers with application to layered media. Zeitschrift für angewandte Mathematik und Physik, 62(6):1131-1142, 2011.

[62] S. Rudykh, K. Bhattacharya, and G. deBotton. Snap-through actuation of thick-wall electroactive balloons. Int J Non Linear Mech, 47(2):206-209, 2012.

[63] S. Rudykh, A. Lewinstein, G. Uner, and G. deBotton. Analysis of microstructural induced enhancement of electromechanical coupling in soft dielectrics. Appl Phys Lett, 102(15):151905, 2013.

[64] J. Schröder, P. Neff, and D. Balzani. A variational approach for materially stable anisotropic hyperelasticity. International Journal of Solids and Structures, 42(15):4352-4371, 2005.

[65] J. Schröder and P. Neff. Invariant formulation of hyperelastic transverse isotropy based on polyconvex free energy functions. Int J Solids Struct, 40(2):401-445, 2003.

[66] G. Shmuel and G. deBotton. Band-gaps in electrostatically controlled dielectric laminates subjected to incremental shear motions. J Mech Phys Solids, 60(11):1970-1981, 2012.

[67] G. Shmuel and G. deBotton. Corrigendum to "band-gaps in electrostatically controlled dielectric laminates subjected to incremental shear motions" [journal of the mechanics and physics of solids 60 (2012) 1970-1981]. J Mech Phys Solids, 105:21-24, 2017.

[68] B. Shrimali, V. Lefèvre, and O. Lopez-Pamies. A simple explicit homogenization solution for the macroscopic elastic response of isotropic porous elastomers. Journal of the Mechanics and Physics of Solids, 122:364-380, 2019.

[69] M. Šilhavý. A variational approach to nonlinear electro-magneto-elasticity: Convexity conditions and existence theorems. Mathematics and Mechanics of Solids, 23(6):907-928, 2017.

[70] H. Stoyanov, M. Kollosche, S. Risse, D. N. McCarthy, and G. Kofod. Elastic block copolymer nanocomposites with controlled interfacial interactions for artificial muscles with direct voltage control. Soft Matter, 7(1):194-202, 2011.

[71] Z. Suo, X. Zhao, and W. Greene. A nonlinear field theory of deformable dielectrics. J Mech Phys Solids, 56(2):467-486, 2008.

[72] L. Tian, L. Tevet-Deree, G. deBotton, and K. Bhattacharya. Dielectric elastomer composites. J Mech Phys Solids, 60(1):181-198, 2012.

[73] R. A. Toupin. Stress tensors in elastic dielectrics. Arch Ration Mech Anal, 5(1):440-452, 1960.

[74] R. Toupin. The elastic dielectric. Indiana Univ. Math. J., 5:849-915, 1956. ISSN 0022-2518.

[75] D. K. Vu, P. Steinmann, and G. Possart. Numerical modelling of non-linear electroelasticity. Int J Numer Methods Eng, 70(6):685-704, 2007.

[76] D. Vu and P. Steinmann. On 3-d coupled BEM-FEM simulation of nonlinear electroelastostatics. Comput Methods Appl Mech Eng, 201-204:82-90, 2012. 
[77] B. Wu, W. Zhou, R. Bao, and W. Chen. Tuning elastic waves in soft phononic crystal cylinders via large deformation and electromechanical coupling. Journal of Applied Mechanics, 85(3), 2018.

[78] Q. M. Zhang, H. Li, M. Poh, F. Xia, Z.-Y. Cheng, H. Xu, and C. Huang. An all-organic composite actuator material with a high dielectric constant. Nature, 419(6904):284-287, 2002.

[79] X. Zhao and Q. Wang. Harnessing large deformation and instabilities of soft dielectrics: Theory, experiment, and application. Applied Physics Reviews, 1(2):021304, 2014. 